\title{
Quantum simulation of the Hubbard model
}

\section{Citation}

Chiu, Christie Shinglei. 2019. Quantum simulation of the Hubbard model. Doctoral dissertation, Harvard University, Graduate School of Arts \& Sciences.

\section{Permanent link}

http://nrs.harvard.edu/urn-3:HUL.InstRepos:42106921

\section{Terms of Use}

This article was downloaded from Harvard University's DASH repository, and is made available under the terms and conditions applicable to Other Posted Material, as set forth at http:// nrs.harvard.edu/urn-3:HUL.InstRepos:dash.current.terms-of-use\#LAA

\section{Share Your Story}

The Harvard community has made this article openly available.

Please share how this access benefits you. Submit a story.

\section{Accessibility}




\title{
Quantum simulation of the Hubbard model
}

\author{
A DISSERTATION PRESENTED \\ BY \\ Christie Shinglei Chiv \\ TO \\ The Department of Physics \\ IN PARTIAL FULFILLMENT OF THE REQUIREMENTS \\ FOR THE DEGREE OF \\ Doctor of PhILOSOPHY \\ IN THE SUBJECT OF \\ Physics \\ HARVARD UnIVERSITY \\ Cambridge, Massachusetts \\ MAY 2019
}


(C) 2019 - Christie Shinglei Chiu ALL RIGHTS RESERVED. 


\title{
Quantum simulation of the Hubbard model
}

\begin{abstract}
The Hubbard model was originally proposed in 1963 as a simple model to describe electrons in a solid. Today, the model is widely believed to capture the physics of cuprate materials, which exhibit phenomena that are not yet well understood such as unconventional superconductivity. However, the model itself is still incredibly challenging to solve on a fundamental level due to strong correlations in the many-body system.

We use a quantum gas microscope of fermionic neutral lithium atoms in an optical lattice to implement the Hubbard model and gain physical insight into the underlying many-body physics. Through independent control of particle tunneling, on-site interaction, and site-resolved potentials, plus site-resolved projective measurement, this platform offers a clean and tunable system for indepth study.

In this thesis, I describe two pathways toward probing the physics of the Hubbard model. First, I discuss our experimental implementation and characterization of a quantum state engineering technique to realize lower-entropy many-body states beginning from an ultra-low entropy band insulator. We find that although it is insufficient in its current form, the technique is highly promising and motivates the development of more sophisticated elements. Second, I share our work performing quantum simulation of the doped Hubbard model, where we find new ways to examine microscopic theories of how doped holes behave in a quantum antiferromagnet. These efforts reflect the ongoing challenges and successes toward achieving full-scale quantum simulation of the Hubbard model with ultracold atoms.
\end{abstract}




\section{Contents}

I INTRODUCTION I

2 Theory AND Background 6

2.I The Hubbard model . . . . . . . . . . . . . . . . . . 7

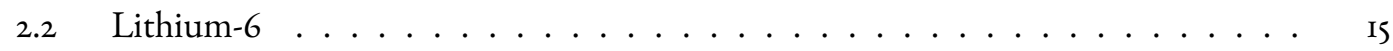

$2.3 \quad$ Ultracold atom studies of the Hubbard model . . . . . . . . . . . . . $\quad$ I7

2.4 Quantum gas microscopy and condensed-matter tools . . . . . . . . . . . . I8

3 Quantum State engineering 24

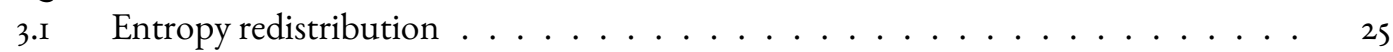

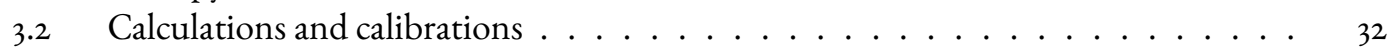

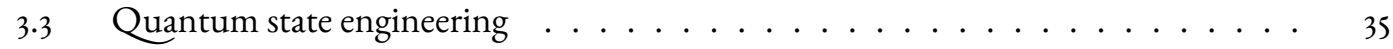

3.4 Experimental implementation . . . . . . . . . . . . . . . . . . 39

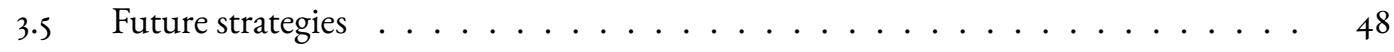

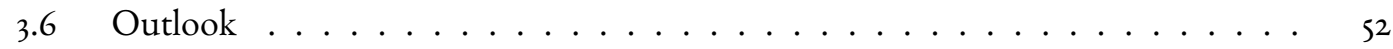

4 STRING PATTERNS 54

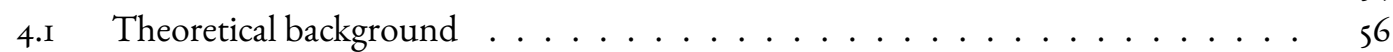

4.2 Experimental dataset . . . . . . . . . . . . . . . . . . . 69

4.3 Analysis methodology . . . . . . . . . . . . . . . . 74

4.4 String-pattern observables $\ldots \ldots \ldots \ldots \ldots \ldots \ldots \ldots$

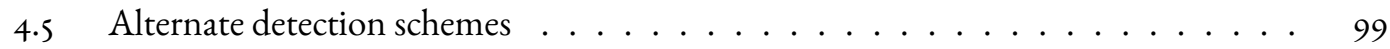

4.6 Conventional spin observables $\ldots \ldots \ldots \ldots \ldots \ldots \ldots$ IO2

4.7 Hole-hole correlations . . . . . . . . . . . . . . . . . . . I04

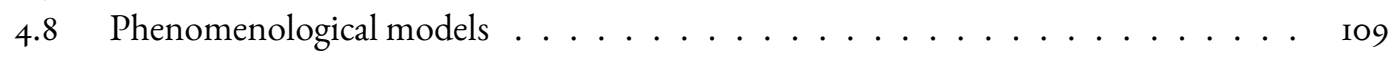

4.9 Expansion of pattern detection algorithms . . . . . . . . . . . . II2

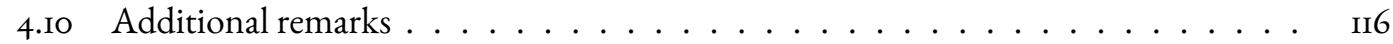

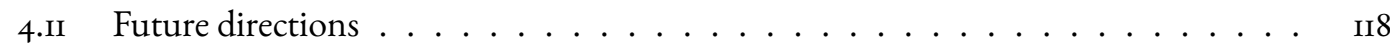

5 Conclusion

$\begin{array}{ll}\text { REFERENCES } & 134\end{array}$ 
TO MY FAMILY:

Steve, Michell, and Michael. 


\section{Acknowledgments}

MANY INDIVIDUALS have deeply enriched my graduate school experience in ways that the physics alone, as incredible as it was, could not have.

Markus Greiner made all of this possible by offering me a position in his lab. I am incredibly appreciative of his investment in my training, which began with a conference sponsorship even before I started in his lab and continued throughout my graduate career with him giving me many opportunities to give invited talks and pushing me to the highest standards of science. I very much enjoyed gaining an intuition for quantum many-body systems from Markus, discussing colorbars with him, and being blown away by his mastery of optics.

From the team-oriented nature of our work, it's no surprise that I spent the most time with and learned the most from my labmates in all three of Markus' experiments. They taught me so much about not only atomic physics, optics, and electronics, but also about questioning everything and being critical. In particular, I must thank Florian, Sebastian, Max, and Anton for their patience in the face of my unending questions when I first joined Lithium lab. A big thanks also to Daniel Greif, Geoffrey Ji, Muqing Xu, and Justus Brüggenjürgen for their patience in the face of my unending questions as the senior grad student of Lithium lab. I will miss our Greiner group traditions-our Secret-Santa-turned-White-Elephant holiday gift exchange, our group hikes, and our neverending banter, to name a few.

So much happens behind the scenes to make sure that things actually get done. Carol, Lisa, Samantha, Clare, and Jacob are amazing at making sure that things run smoothly, everything from progress towards a degree to building a cohesive Physics department community. I also want to thank Stan, one of the first individuals I worked closely with when I first started at Harvard, for his machining expertise; I am so grateful that I learned to machine from him. These individuals are all so amazing at their jobs, but they do so much more - they're always looking out for others, they genuinely care so much, and they welcomed me into the community from day one-and that's something I'll never forget.

I am so fortunate (as an introvert) to have found so many friends among my fellow physicists in 
the department. Anne is one of the kindest, most thoughtful individuals I've ever met, and I'm so happy that we really got to know each other on a group backpacking trip and started an amazing friendship. Annabelle and Fabian were wonderful collaborators but also wonderful friends; their excitement and optimism is simply unparalleled. I also want to thank Ana, Anj, Elana, Ellen, Grace, Joyce, and Yanting for all of our fun (and serious) conversations and food dates.

Much of the work I did outside the lab was just as educational as the work I did in the lab. My fifth year, I had the opportunity to participate in the Harvard Horizons program, and I had so much fun getting to know the other seven Harvard Horizons scholars and discussing their work. I thank them all, as well as Mara, Lindsey, Marlon, and Pamela, for such a wonderful experience. I also spent five years as a First-year Proctor for Harvard College, and loved working with all five of my entryways and their Peer Advising Fellows: Stoughton South Class of 2018, Hollis North Class of 2019, Hollis North Class of 2020, Pennypacker 4 Class of 2021, and Pennypacker 4 Class of 2022. Through helping each of them navigate their first year of college, I learned how to navigate my own challenges. Through learning more about them and their stories, I learned more about the world and was inspired by their accomplishments. I also thank my fellow proctors and Mike, Catherine, and Dwight, the three Resident Deans of Freshmen I worked with, for their constant support in both my role as a Proctor and my role as a grad student.

An enormous thanks to Janet Conrad and Nergis Mavalvala, two mentors and role models from my undergraduate days whose guidance was instrumental in my development as a physicist and preparation for graduate school. Their kind words and advice have remained with me throughout my graduate career and will stay with me as I head into my postdoc.

Day to day, Brian and Velvet were constant sources of happiness (and cuteness, in the case of Velvet). I am so unbelievably lucky to have gotten to know Brian through ballroom and for all of our adventures since, and to have adopted Velvet and gotten to know her coy yet silly and affectionate personality.

Most importantly, I am forever indebted to my parents, Steve and Michell Chiu, and my brother, Michael Chiu. Their generosity, unwavering support, and stubborn insistence that I can accomplish anything has given me so much strength and positivity. I thank them for allowing me to dream big when I was little, for celebrating with me in my successes, and for consoling me in my failures.

And a big thanks to you, the reader, for taking the time to read (at least some of) my dissertation. While I (on average) enjoyed writing this body of work, without you it loses much of its meaning. I hope you find it interesting and helpful. 


\section{1}

\section{Introduction}

IT's JANUARY 27, 1986, and two experimentalists in Zurich have witnessed a sharp drop in the resistance of their material at around ${ }_{3} 3$ Kelvin. The record at the time for the highest superconducting transition temperature was 23 Kelvin, which had been the case for the past 12 years. For these two experimentalists, however, their material was not a cubic niobium compound like many of the su- 
perconductors discovered before- rather, it was a layered copper oxide. Three months later, and they had improved the ratios of barium, lanthanum, copper, and oxygen in their material and how it was annealed to achieve a transition temperature of 35 Kelvin $^{\mathrm{I}}$. Not only had K. Alex Müller and J. Georg Bednorz beaten the record for the highest transition temperature, but they had also created an entirely different type of superconductor: the first "high-temperature" superconductor. This achievement won them the 1987 Nobel Prize in Physics.

IT's APRIL 18, 20I4, and two graduate students in Cambridge, Massachusetts rearrange the optics of their experiment to once again try and image single atoms. Four other groups around the world are attempting the same feat. One student clicks "Run Scan", and the experiment comes alive, cooling and loading a cloud of lithium atoms into a periodic array of traps as it had done tens of thousands of times before. An image updates on the screen: noisy, but with several bright round blobs. These were atoms-poorly imaged atoms, which couldn't even compare to the rubidium quantum gas microscopes, but unlike its predecessors these were fermionic atoms. It took the group of five-Florian Huber, Maxwell Parsons, Anton Mazurenko, Sebastian Blatt, and myself_-another year to perfect the high-fidelity imaging of fermionic atoms, in concert with the other groups worldwide, and our scientific community nicknamed 2015 "the year of Fermi gas microscopy". This achievement was a huge step in cold-atom studies of the Hubbard model and, possibly, in achieving a microscopic understanding of the high-temperature superconductivity discovered almost thirty years prior. 


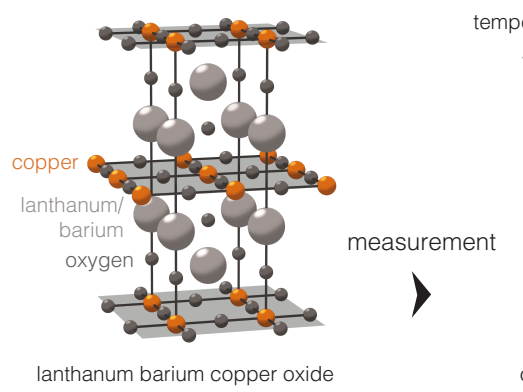

lanthanum barium copper oxide approximate Hamiltonian

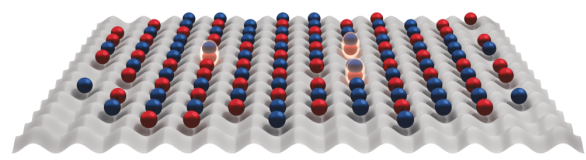

ultracold lithium-6 in an optical lattice Hubbard model
Figure 1.1: Quantum simulation with the Hubbard model. Lathanum barium copper oxide (LBCO) was the first high-temperature superconductor discovered. It is one in a family of cuprate materials which exhibit this phenomenon. While these materials have been studied extensively and phase diagrams have been mapped out (here a representative schematic is shown), many open questions remain. The underlying physics stems from the valence electrons of the copper atoms which are arranged in two-dimensional (2D) planes. Therefore, we use ultracold neutral atoms in a 2D plane of an optical lattice to simulate these electrons and gain physical insights.

High-TEMPERATURE SUPERCONDUCTORS are one example of strongly correlated quantum sys-

tems, systems of many quantum particles which cannot be described by single-particle or mean-field theories. Despite decades of intense theoretical, numerical, and experimental work, we do not have a unified microscopic model for these superconductors' macroscopic behavior, nor a formalism for treating strongly correlated quantum systems more generally. Exact calculations and simulations become exceedingly challenging, even on today's state-of-the-art supercomputers. New theories and numerical computation may be helpful, but ultimately must be experimentally tested. The creation of new materials for investigation are limited by existing manufacturing technologies, and measurements on real materials are time- or spatially averaged due to the fast dynamics of and small distances between the electrons.

Ultracold atom experiments may be able to provide a complementary approach. This platform allows for the creation of quantum states in a clean, tunable environment and for readout with high 
spatial and temporal resolution. By loading a cold atomic gas into an optical lattice (a periodic array of traps formed by laser light), such experiments can realize the Hubbard model ${ }^{2}$. The Hubbard model ${ }^{3,4,5}$ is a minimal model thought to capture the physics of cuprate high-temperature superconductors, so by studying it with ultracold atoms we may reveal new physical insights on a condensed matter phenomenon with far-reaching technological applications. More broadly speaking, we are implementing a quantum simulator (see figure I.I): using one quantum system to probe the physics of an analogous quantum system, as introduced by Richard Feynman in $1982^{6}$. The advantage is that we approach the problem of solving classically computationally intractable problems by creating an accurate physical model which is easier to control and measure.

At the same time, the Hubbard model is a fascinating problem in its own right. It is described by two straightforward terms, yet beyond the one-dimensional case it remains analytically unsolved with many open questions on its phase diagram. Numerics of the two-dimensional Hubbard model indicate the existence of a $d$-wave superconducting phase ${ }^{7}$, but this has yet to be experimentally shown. As for the pseudogap phase seen in the cuprates, this is also believed to be supported by the Hubbard model. However, it is not yet agreed upon whether the pseudogap arises from superconducting fluctuations ${ }^{8,9, \mathrm{ro}}$, is a competing phase with superconductivity ${ }^{\mathrm{II}, \mathrm{I2}, \mathrm{r3}}$, or may be something different altogether ${ }^{\mathrm{I} 4}$. In other words, as simple as the Hubbard model seems, it exhibits strongly correlated quantum physics which cannot be completely described with current analytical or numerical techniques. And so our investigation of the Hubbard model with ultracold atoms is incredibly rich in that it has deep connections to condensed matter, quantum computation, and fundamental quantum physics. 
In the group of Markus Greiner, we achieved high-fidelity single-site imaging of fermionic lithium in April 20I5 ${ }^{15,16,17}$. Later that year, we optimized our experimental sequence and installed a gradient coil for efficient evaporation; with these improvements we were able to reduce our temperatures and realize a Mott insulator of fermions ${ }^{\mathrm{I} 8, \mathrm{I7}}$. After introducting spin-selective imaging and digital micromirror devices to reshape the potential, we were able to measure the spin correlation function ${ }^{\mathrm{I} 9 \mathrm{I} 7}$ and realize a cold-atom Hubbard antiferromagnet (AFM) under the microscope ${ }^{20,21}$, respectively. This brings us to the work presented in this thesis.

The thesis proceeds as follows:

- Chapter 2 reviews the atomic, lattice, and Hubbard model physics behind the material presented in Chapters 3 and 4.

- Chapter 3 presents our implementaton of a quantum state engineering protocol with the goal of reaching lower-entropy states. It includes the content of the publication:

C.S. Chiu, et. al. PRL I2O, 243201 (2018) 22

with contributions from co-authors Geoffrey Ji, Anton Mazurenko, Daniel Greif, and Markus Greiner.

- Chapter 4 details the saga of our explorations to find a new real-space observable which contains useful information beyond conventionally used observables, and to scrutinize microscopic theories of the doped Hubbard model in our currently accessible temperature range. It includes the content of the manuscript:

C.S. Chiu, et. al. arXiv:I812.03584 (2018) 23 with contributions from co-authors Geoffrey Ji, Annabelle Bohrdt, Muqing Xu, Michael Knap, Eugene Demler, Fabian Grusdt, Markus Greiner, and Daniel Greif.

- Chapter 5 leaves the reader with an update on the current status of the experiment and outlook on the exciting future of Fermi gas microscopy.

Let's begin! 


\section{2 \\ Theory and background}

OUR EXPERIMENTS USE AN ULTRACOLD GAS OF FERMIONIC LITHIUM, Lithium-6, in a balanced spin mixture of the lowest two hyperfine states. We load this gas into the lowest band of a single two-dimensional $(2 \mathrm{D})$ plane of a $3 \mathrm{D}$ optical lattice formed by retroreflected laser beams. With the lattice optical power and a magnetic field bias, we can independently control how easily atoms tun- 
nel to neighboring lattice sites and the on-site contact interaction for two atoms on one site. We can also reshape the overall shape of the lattice potential by projecting spatial patterns of light through the microscope objective. For readout we freeze the atom distribution by quickly ramping up the laser power, enabling measurements of both equilibrium and non-equilibrium physics. One spin species can be optionally removed prior to imaging, or the entire atom distribution can be imaged without spin information. The resulting measurements in the parity-projected Fock basis are used to calibrate the experiment and study the Hubbard model.

In this chapter I provide the necessary theory and background to understand and contextualize the work presented in the remaining chapters. I begin with the a primer on the Hubbard model before moving on to how we implement the Hubbard model with ultracold atoms in optical lattices and reviewing previous relevant work. As the quantum science community has been using this experimental platform to study strongly correlated quantum systems for almost two decades now, much of the relevant theoretical background has been written up beautifully in review articles or doctoral theses. Because of this, where appropriate I refrain from including repeat derivations and reference particularly well-written works on the mathematical formalism.

\section{I The Hubbard MOdel}

The Hubbard model is defined by the following Hamiltonian:

$$
\hat{\mathcal{H}}=-t \sum_{\sigma=\uparrow, \downarrow} \sum_{\langle i, j\rangle}\left(\hat{c}_{\mathrm{i}, \sigma}^{\dagger} \hat{c}_{\mathrm{j}, \sigma}+\text { h.c. }\right)+U \sum_{\mathbf{j}} \hat{c}_{\mathrm{j}, \uparrow}^{\dagger} \hat{c}_{\mathrm{j}, \uparrow} \hat{c}_{\mathrm{j}, \downarrow}^{\dagger} \hat{c}_{\mathrm{j}, \downarrow}
$$




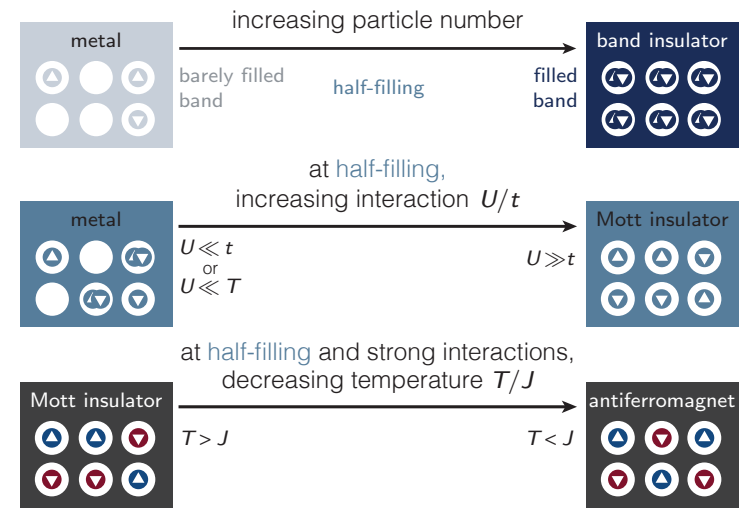

Figure 2.1: Introduction to the Hubbard model. The basics of the Hubbard model can be understood by considering its limits. In the limit of very few particles or very many particles (a filled band), metals and band insulators can be realized, respectively. At half-filling, if the tunneling $t$ or temperature $T$ dominate, again we have a metal; if the interaction $U$ dominates (for repulsive interactions $U>0$ ), we realize a Mott insulator. At intermediate to strong interactions, for sufficiently low temperature an antiferromagnet emerges.

describing spin-I/2 fermions $\hat{c}_{\mathrm{j}, \sigma}$ with spin $\sigma$ in the lowest band of a lattice; here we consider a $2 \mathrm{D}$ square lattice. The first term describes tunneling of amplitude $t$ between adjacent sites $i$ and $j$. The second term includes on-site interactions of strength $U$ between fermions of opposite spin. There is a mapping between the attractive and repulsive Hubbard models with $U<0$ and $U>0{ }^{24}$; here we focus on repulsive interactions.

As depicted in figure 2.I, one can gain an intuition for the model by first considering the role of particle number, related to the chemical potential by the equation of state ${ }^{18,25}$. First, for high particle number, we fill the lattice with two particles per site and the band is completely filled. This phase has no charge mobility and is the band insulator. Otherwise, the band is not filled and many states are available with a relatively high density of states. As a result, particles can delocalize across or tunnel between sites. This is the metallic phase, marked by high charge mobility and large charge fluctuations on every site.

When the band is half-filled with one particle per site, we additionally consider the three energy scales of the Hubbard parameters $t$ and $U$, as well as the temperature $T$. Here I will take units where 
$k_{\mathrm{B}}=\hbar=1$ for ease of comparing temperatures and frequencies as energies. If the tunneling or temperature dominates, again we have a metal. By contrast, for large repulsive interactions the interaction energy cost for having two particles on one site becomes prohibitively high. In this situation particles will instead sit one to a site, with vanishing charge mobility and vanishing charge fluctuations. This is a Mott insulating state, whose insulating character is due to strong interactions between the particles.

The strongly correlated physics becomes evident once spin is considered. For example, in the Mott insulator the particles cannot tunnel to neighboring sites: at an average of one particle per site, this is either energetically unfavorable from the strong interaction energy (for two opposing spins) or forbidden by Pauli blocking (for two of the same spin). If the temperature is sufficiently low, then particles can satisfy their tendency to delocalize by sitting next to particles of opposite spin, and antiferromagnetic spin correlations emerge ${ }^{26}$. The dominant process then becomes superexchange, a second-order process where two particles of opposing spin on neighboring sites switch positions. This has energy $J=4 t^{2} / U$, termed the superexchange energy, and gives the characteristic energy scale for antiferromagnetic spin correlations: $T<J$. I also note that in discussing the physics of the Hubbard model, we care about relative energy scales, rather than absolute ones, simply because all of the energies can be rescaled and yield the same physics. As such, we consider the interaction and superexchange energies in units of the tunneling, and the temperature either in units of the tunneling or in units of the superexchange energy.

Antiferromagnetic spin correlations can be characterized by the two-particle spin correlator 
$C_{s}(d)$, given by

$$
C_{s}(|\mathrm{~d}|) \equiv \frac{\left\langle\hat{S}_{\mathrm{i}}^{z} \hat{S}_{\mathrm{i}+\mathrm{d}}^{z}\right\rangle-\left\langle\hat{S}_{\mathrm{i}}^{z}\right\rangle\left\langle\hat{S}_{\mathrm{i}+\mathrm{d}}^{z}\right\rangle}{S^{2}}
$$

between two sites i and j separated by distance d, where $\hat{S}_{\mathrm{i}}^{z}$ is the spin- $S$ operator on site i and $S=$ $1 / 2$. This quantity tells us how frequently any two spins a distance $d$ apart are aligned or antialigned with each other beyond uncorrelated coincidences. Because the square lattice is bipartite, we can split it into two sublattices $A$ and $B$ where no sites in $A(B)$ are nearest neighbors with other sites in $\mathrm{A}$ (B). Antiferromagnetic spin correlations then correspond to negative values of $C_{s}(d)$ if $d$ is such that sites $\mathrm{i}$ and $\mathrm{j}$ are on different sublattices, and positive values if $d$ is such that they are on the same sublattice. Often I will show the sign-corrected spin correlator $\tilde{C}_{s}(d)$ which takes on positive values for antiferromagnetic correlations. A correlation length can be extracted from the decay of correlations with distance at large distances, where an antiferromagnet is realized if the correlation length is on the order of the system size.

One can also consider the staggered magnetization operator $\hat{m}^{z}$, the order parameter for the antiferromagnet, for a system size of $N$ sites:

$$
\hat{m}^{z}=\frac{1}{N} \sum_{\mathrm{i}}(-1)^{\|\mathrm{i} \mid\|} \frac{\hat{S}_{\mathrm{i}}^{z}}{S}
$$

where $\|\mathrm{i}\|$ denotes the $\ell_{1}$ norm of $\mathrm{i}$, that is, the sum $|x|+|y|$ for vector $\mathrm{i}=\left(\begin{array}{c}x \\ y\end{array}\right)$. This quantity describes the overlap of the spatial spin distribution with a checkerboard pattern. In other words, if one spin species sits on one sublattice, while the other spin species sits on the other sublattice, the 
resulting staggered magnetization has a magnitude of $\mathrm{I}$. Any deviation from this pattern decreases the staggered magnetization.

\section{I.I The Hubbard antiferromagnet}

For the $2 \mathrm{D}$ Hubbard model (as well as other models with continuous symmetries and sufficiently short-range interactions), the Mermin-Wagner-Hohenberg theorem states that in the thermodynamic limit there is no long-range spin order except at temperature $T=0^{27,28}$. However, in our experiments the system size is always finite. Because of this, for sufficiently low temperature $T<J$ and at half-filling, antiferromagnetic spin correlations can still extend over the entire system, with nonzero spin correlations at distances equal to the system size. The accompanying correlation length becomes comparable to the system size, growing rapidly with decreasing temperature ${ }^{20}$. Here, a Hubbard antiferromagnet is realized.

There are several additional characteristics of Hubbard antiferromagnets to note. First, we do not apply a symmetry breaking field to the $\mathrm{SU}(2)$-symmetric Hubbard Hamiltonian. As a result, there is no preferred symmetry breaking direction in our snapshots of the antiferromagnet. This manifests in both the spin correlation function and the staggered magnetization. In the full counting statistics of the staggered magnetization, where it is measured for many experimental realizations, some outcomes yield values of the high staggered magnetization and checkerboard-like spin distributions. However, many more yield low values. Here one can think of the $S U(2)$ symmetry resulting in the random direction of a staggered spin ordering vector $\hat{\mathrm{m}}=\left(\hat{m}^{x}, \hat{m}^{y}, \hat{m}^{z}\right)$ with measurements accessing only $\hat{m}^{z}$. As a result, the symmetry contributes to the spread of measured values. Similarly, 
in the spin correlation function this symmetry results in a suppression of the correlation strength.

Second, the Hubbard antiferromagnet also exhibits singlet character. This can be understood through considering a double well with two particles, one of each spin. Here, the ground state is a spin singlet. As we move to a $\mathrm{ID}$ chain, the second-order superexchange continues to favor singlets, but now each site has two neighbors instead of one. This additional degree of freedom results in a relative decrease in singlet character compared to the o $\mathrm{D}$ (double-well) case. In $2 \mathrm{D}$, some of the singlet character persists, but is again reduced from the increase of coordination number to four. This singlet character can be seen in the spin correlation function; while spin correlations exponentially decay with distance for large distances, the strength of the nearest-neighbor spin correlator is strongly enhanced relative to this exponential character. The enhancement can be understood by noting that regardless of the basis in which a singlet state is measured, it always exhibits an anticorrelated spin pair. By contrast, the spin correlations at longer distances do not exhibit this entanglement, and have the suppressed correlation strength from the projective measurement in the Fock basis. However, take note that this measurement alone is not sufficient to prove the existence of singlets.

Third, Hubbard antiferromagnets contain doublon-hole pairs if the interaction energy is not infinite. Two opposite spins on neighboring sites can undergo virtual tunneling processes where both particles sit on the same site next to an unoccupied site. The probability of imaging such doublon-hole configurations roughly scales as the superexchange energy and vanishes in the infiniteinteraction limit. This can also be seen by considering a double-well picture. For $U>>t$, the ground state is the singlet state. For more intermediate values of $U / t$, the ground state sees an ad- 
mixture of the singlet state and the doublon-hole pair, with an amplitude of the latter of approximately $2 t / U$ resulting in a detection probability given by the superexchange. Of course, in the extreme case of low $U / t$, charge fluctuations dominate and the Mott insulator no longer exists. We can image doublon-hole pairs in the antiferromagnet by "freezing" out the dynamics of the system sufficiently quickly such that no additional dynamics occur during this process. In other words, we quickly suppress tunneling between neighboring sites so that our image accurately reflects the state at our chosen Hubbard parameters.

\section{I.2 APPROXIMATE SPIN MODELS}

In certain limits, the Hubbard model can be approximated by more easily computable spin models where the Hilbert space is significantly smaller or where there is no "negative sign problem" 29. Here I discuss two which pertain to the strong coupling limit $U / t>>1$. At half-filling, strong interactions fix exactly one atom to a site and only superexchange processes occur, which can be described by the Heisenberg model. Away from half-filling, particles can continue to tunnel and the $t-J$ Hamiltonian corresponds to this model. Both of these models are relevant to the discussions in Chapter 4.

\section{HEISENBERG MODEL}

The Heisenberg model is described by the Hamiltonian:

$$
\hat{\mathcal{H}}_{\text {Heisenberg }}=J \sum_{\langle i, j\rangle} \hat{S}_{i} \cdot \hat{S}_{j}
$$


Here spins are fixed to exactly one per site, with the second-order superexchange now written as a nearest-neighbor spin interaction $J$ and $J>0$. This corresponds to the Hubbard model in the limit of strong interactions $U / t>>1$ and a density of one atom per site, $n=1^{26,30,31}$. Notice that this Hamiltonian preserves the $S U(2)$ symmetry of the Hubbard model. At the same time, the Hilbert space grows approximately exponentially more slowly with system size compared to the Hubbard model. As a quick back-of-the-envelope calculation, if we take an average of one particle per site and a balanced spin population, for $N$ sites under the Hubbard model each spin state has ${ }_{N} C_{N / 2}=N ! /(N / 2) !^{2}$ configurations, for a total of $\left(N ! /(N / 2) !^{2}\right)^{2}$ states. By contrast, under the Heisenberg model we have exactly one particle per site. This is equivalent to considering the number of configurations for a single spin state and requiring that the remaining sites are occupied with the other spin, giving us a total of $N ! /(N / 2) !^{2}$ states. Under Stirling's approximation the

Hilbert space size for the Hubbard model at half-filling grows $\sqrt{2 / \pi N} 2^{N}$ times more quickly than that for the Heisenberg model. This reduction in Hilbert space makes numerical treatments of the Heisenberg model significantly more straightforward, but is limited to states at half-filling.

\section{$t-J$ MODEL}

If we again consider the Hubbard model in the strongly interacting limit but allow particles to tunnel, we can instead obtain the $t-J$ Hamiltonian ${ }^{32,31}$ :

$$
\hat{\mathcal{H}}_{t-J}=-t \sum_{\sigma=\uparrow, \downarrow} \sum_{\langle\mathrm{i}, \mathrm{j}\rangle}\left(\hat{c}_{\mathrm{i}, \sigma}^{\dagger} \hat{c}_{\mathrm{j}, \sigma}+\text { h.c. }\right)+J \sum_{\langle\mathrm{i}, \mathrm{j}\rangle}\left(\hat{\mathrm{S}}_{\mathrm{i}} \cdot \hat{\mathrm{S}}_{\mathrm{j}}-\frac{1}{4} \hat{n}_{\mathrm{i}} \hat{n}_{\mathrm{j}}\right)
$$


where the number operator $\hat{n}_{\mathbf{i}}=\sum_{\sigma} \hat{c}_{\mathrm{i}, \sigma}^{\dagger} \hat{c}_{\mathrm{i}, \sigma}$ gives the particle number on site i. This Hamiltonian, too, preserves the $S U(2)$ symmetry of the Hubbard model. In addition, there are again no doubly occupied sites because we are in the strongly interacting limit. As a result, at half-filling the size of the Hilbert space is equivalent to that of the Heisenberg model. Away from an average of one particle per site, the $t$ - $J$ model still sees a significant reduction in Hilbert space compared to the Hubbard model, but as soon as there is more than one dopant the negative sign problem comes into play, just as for the Hubbard model.

\subsection{Lithium-6}

Lithium- 6 was chosen with both technical and scientific considerations in mind. Alkali atoms are in general good candidates for ultracold atom experiments because of their hydrogenic electronic structure with relatively few energy levels to consider. Of the alkali atoms, only lithium and potassium have stable fermionic isotopes. While both elements have by now been successfully imaged via fermi gas microscopy $33,34,16,35$, a priori the almost 7 -fold lighter mass of lithium appealed to us. The energy scale of particles in a lattice is given by the recoil energy $E_{r}=\hbar^{2} k^{2} / 2 m$, where $\hbar k$ is the lattice photon momentum and $m$ is the particle mass. As a result, in absolute units both the tunneling $t$ and the superexchange $J=4 t^{2} / U$ timescales for lithium are almost 7 times faster than that of potassium. This is advantageous because if the physics timescales are too long, experiments become limited by non-lattice-physics timescales, namely background gas collisions.

Atomic scattering properties are also significant, as they dictate thermalization dynamics in bulk 


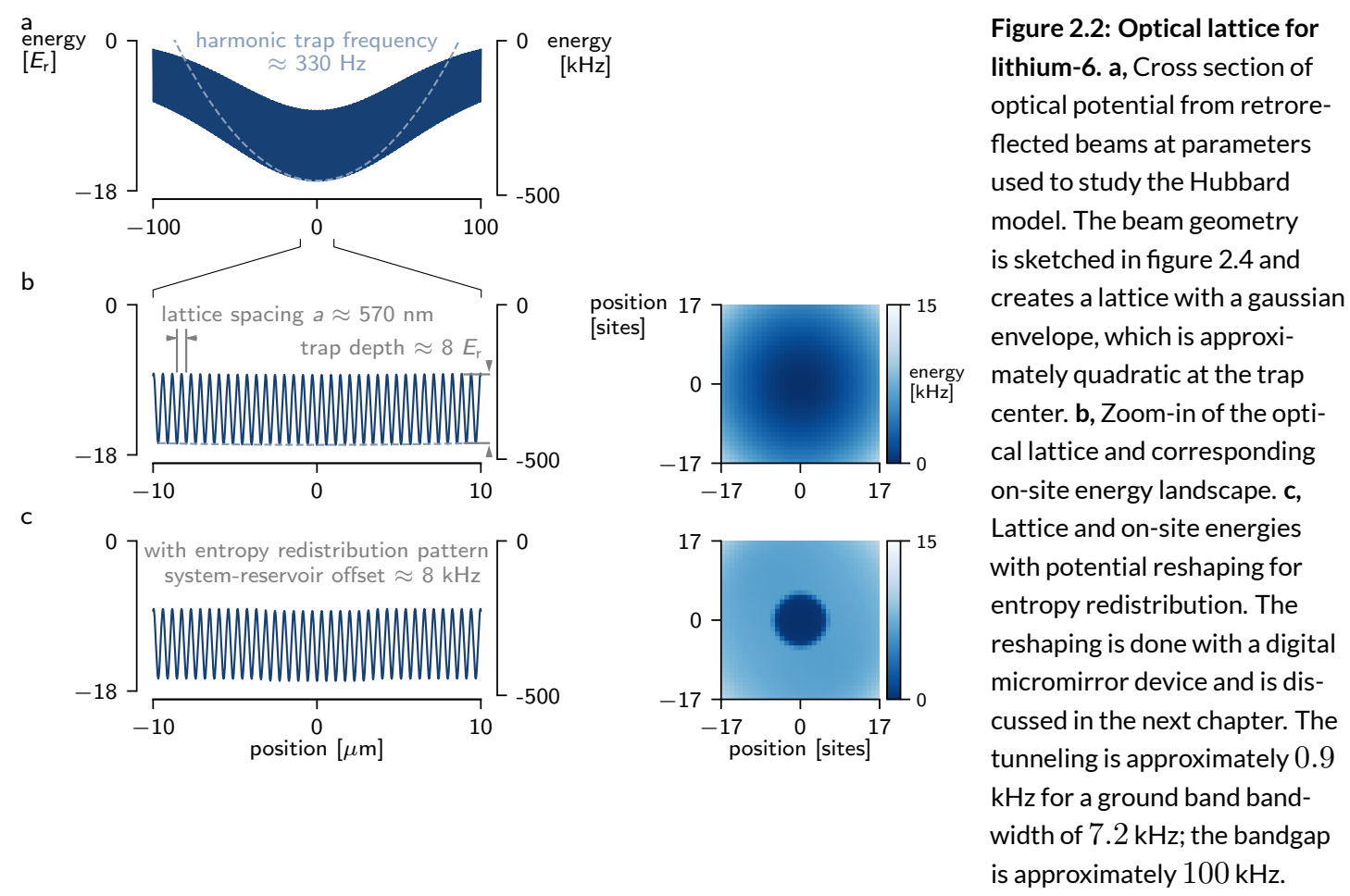

and determine on-site interaction energies in the lattice. In cold atom experiments the scattering length can be tuned by sitting close to a Feshbach resonance, a strong coupling between the open channel of two colliding atoms and a closed molecular channel ${ }^{36}$. The Feshbach resonances in Lithium are well-suited for our experimental needs. For example, there is a Feshbach resonance for the lowest two hyperfine states at approximately 834 Gauss which is about $300 \mathrm{G}$ wide. The scattering length sees a zero crossing at approximately $528 \mathrm{G}$ and a local minimum of $-280 a_{0}$ at $356 \mathrm{G}$. Furthermore, these Feshbach resonances have been very carefully measured, to an accuracy better than $7 \times 10^{-4}$ of the width ${ }^{37}$. This enables a high degree of control over the atomic scattering properties by tuning the magnetic field bias, enabling efficient evaporation and high tunability over the 
Hubbard on-site interaction energy.

We implement the Hubbard Hamiltonian by loading lithium atoms into the lowest band of a 2D square optical lattice. The lattice light, being spatially varying and off-resonant, creates a spatially varying ac Stark shift and therefore an optical dipole force ${ }^{38}$. At a laser wavelength of Io64 nm, our lattice is red-detuned and attracts atoms to the intensity maxima. For our two spin states we use the two lowest hyperfine states, $|1\rangle$ and $|2\rangle^{39}$, which interact via $s$-wave scattering from the broad Feshbach resonance. These two ingredients - the lattice and interaction-can be shown to map onto the Hubbard model in the orthonormal Wannier basis ${ }^{40,41,42}$ with two key approximations. First, we assume only the lowest band is occupied. Second, we make the tight-binding approximation, where tunneling only occurs between neighboring sites and particles are sufficiently localized such that there are only on-site interactions. In figure 2.2 I show several to-scale plots depicting the relative lattice energy scales for our typical experimental parameters.

\subsection{Ultracold atom Studies of the Hubbard MOdel}

Many groups have already done amazing work studying the Hubbard model with fermions in optical lattices and trap-averaged measurements. These studies as a whole were made possible with groundbreaking achievements in creating degenerate Fermi gases ${ }^{43,44}$ and implementing the BoseHubbard model with bosons in optical lattices ${ }^{40,45,46,47}$. In 2008, two groups realized Mott insulators of fermions with potassium- $4 \mathrm{O}^{48,49}$, as seen through a vanishing compressibility with atom number or trap confinement, reduction of doubly occupied sites, and emergence of a spectral gap. 


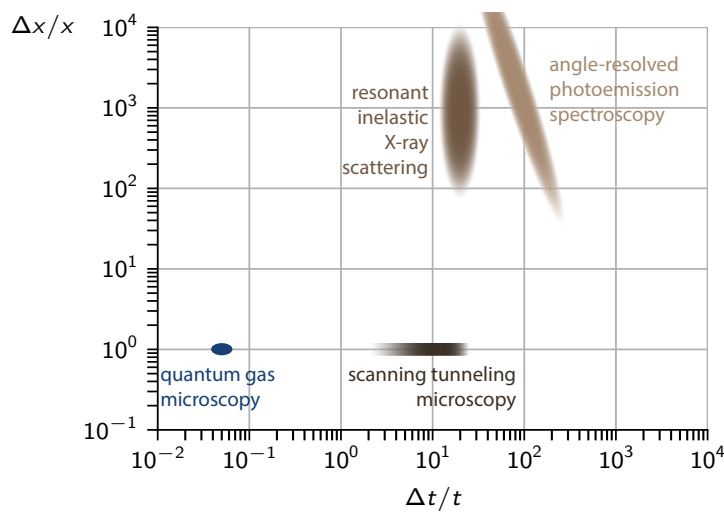

Figure 2.3: Quantum gas microscopy and condensed matter approaches. Here I sketch the length- and timescales to which quantum gas microscopy and several condensed matter tools are sensitive, normalized to the lattice spacing and particle tunneling times. Note that the specifications shown here apply to the time-resolved versions of the condensed matter approaches discussed.

Seven years later, Mott insulators with lithium- 6 were realized with a push away from global observables towards local ones, as a local compressibility was measured by computing the change in density profile over the trap, given the trap shape ${ }^{\text {so }}$. Short-range magnetic correlations have also been measured for fermions in optical lattices through detecting the imbalance between singlets and triplets in $2013^{51}$ or through Bragg scattering of near-resonant light in $2015^{52}$. There is also growing effort to studying extentions to the Hubbard model $^{53}$, for example with more than two spin states ${ }^{54,55}$, varying geometries ${ }^{56,57}$, or long-range interactions ${ }^{58}$.

\subsection{QUantum gaS Microscopy AND CONDENSED-MATTER TOOLS}

By now it seems that quantum gas microscopy is a familiar tool to the atomic physics community, which is remarkable given that it was first demonstrated only about a decade ago. Single atoms of rubidium- $-87^{46,47}$, ytterbium- $174^{59,60}$, potassium- $40^{33,34,35}$, and lithium- $6^{16,61,62}$ have all been imaged, with about ten microscopes currently online and more on the way.

Indeed, quantum gas microscopy has proven to be a powerful tool for accessing strongly corre- 
lated physics due to its high temporal and spatial resolution of approximately 0.05 times the tunneling time and site-resolved resolution. This offers a complement to existing methods for probing real materials, which focus more on energy- and momentum-resolved measurements. Indeed, techniques such as angle-resolved photoemission spectroscopy (ARPES), resonant inelastic X-ray scattering (RIXS), and scanning tunneling microscopy (STM) have been highly successful in revealing the excitation spectra, band dispersion, and density of states of materials.

To begin, photons can be used to probe the band dispersion and Fermi surface of a solid. ARPES uses photons to eject electrons from the material ${ }^{63}$. The energy and momentum of the photoelectron can be measured via its position on a detector after passing through an electrostatic lens. The binding energy of the electron is then given by incoming photon energy, less the measured energy and the electron work function. The electron crystal momentum corresponds directly to the momentum of the measured electron, but only for the component in the plane of the sample due to additional processes (e.g. scattering) which may occur as the electron leaves the material. As a result, ARPES is used to reveal the density of single-particle electronic excitations in momentum space. Additionally, ARPES provides access to measuring the Fermi surface because it only ejects electrons from already-filled states.

With ARPES, easily achievable resolutions are around $8 \mathrm{meV}$ in energy (source-limited) and $0.01 \AA^{-1}$ in momentum (source- and detector-limited), compared to maximal superconducting gap energies around Ios of meV and Brillouin zone widths of about $1.6 \AA^{-1}{ }_{64,65}$. These resolutions have improved greatly over the past few decades and can be pushed to around $3 \mathrm{meV}$ and $0.003 \AA^{-1}$ by using, for example, ultraviolet laser light sources rather than X-rays from a synchrotron source. If 
I convert these specifications to units of the lattice constant $a$ and electron tunneling integral $t$, they correspond to approximately $10^{4} t$ and $5 \times 10^{2} a$.

Time-resolved ARPES has also been developed where an ultrashort pump pulse manipulates the system prior to the probe pulse. The resulting single-particle excitation spectrum and its evolution in time can be analyzed to reveal information about the underlying dynamics after the pump pulse. This addition of femtosecond pump and probe pulses allows for time resolution on the scale of hundreds of femtoseconds, which can get down to several hundred times the tunnelling time as sketched in figure $2.3^{66,67,68}$.

Photons can also be used to excite deeply bound electrons into the valence band, which creates an outgoing photon once the empty state becomes filled by a more loosely bound electron. By measuring the energy, momentum, and polarization of this photon, information about the excitation spectra of the solid can be extracted. This technique is known as RIXS. Because RIXS moves charge around a sample but does not add or remove charge, it accesses charge-neutral excitations including phonons and magnons. RIXS offers an energy resolution of about $30 \mathrm{meV} \approx 10^{5} t$, made challenging by the requirement for a tunable X-ray source, and a momentum resolution of 0.005 times the Brillouin zone width corresponding to a spatial resolution of about $3 \times 10^{2} a^{69}$. However, they also require the high-brilliance radiation sources only offered at synchrotrons. Much in the same way as for ARPES, time-resolved RIXS has also been developed and can achieve temporal resolution of approximately 4 o femtoseconds $\approx 20 t^{70}$.

Alternatively, STM can be used to gain real-space information and image surfaces at the atomic level, offering a resolution of $0.1 \mathrm{~nm}$ in the plane. A conducting tip is brought close to the surface 
and a bias voltage is applied to allow electrons to tunnel between the surface and the tip. This tunnel current can be kept fixed to determine the surface topography. However, because the current also depends on the local density of states, alternatively the tip position can be kept fixed and the current can be used to extract the local density of states by measuring its response to an applied voltage.

Here energy resolutions of approximately $5 \mathrm{meV} \approx 1.6 \times 10^{4} t$ can be achieved, limited by the minimal bias voltage change required to get an acceptable signal to noise ${ }^{71}$. Additionally, not only can electrons tunnel from the surface to the tip, but also vice-versa, so the measurable range does not depend on the Fermi surface. If instead the local density of states is measured across the sample spatially for several bias voltages, a Fourier transform can be taken to extract the energy dispersion curve $^{72,73}$. Then the momentum resolution depends on the sample size, which can easily extend to Ioo sites in each direction, for a resolution of 0.01 times the Brillouin zone. Standard STM techniques can achieve temporal resolution of to to Ioo microseconds, which can be pushed down by a factor of one thousand by scanning over smaller sample sizes for a resolution of about $5 \times 10^{6} t^{74}$. However, pump-probe STM can achieve time resolutions of down to 25 femtoseconds, a time scale just an order of magnitude larger than the tunneling as shown in figure $2.3^{75}$.

Of course there are many more subtle points and features for each of these tools, but this brief summary provides a general context to our work. The most conventional tools were not designed for temporal resolution, and each individual apparatus contains tradeoffs between their momentum and energy resolution capabilities which of course also applies to the tools incorporating temporal resolution. Spin sensitivity has also been developed for each of these tools, but is convolved with both measurement and system parameters and continues to be an active area of research and devel- 
opment.

The broader Fermi gas microscope community has performed a whole host of beautiful studies of the Hubbard model, covering a wide range of parameters. Antiferromagnetic chains have been realized in $\mathrm{I}^{76}$ and antiferromagnets in $2 \mathrm{D}$ of 80 sites have been realized with record-low temperatures of $T / t=0.25(2)^{20}$. The role of spin imbalance has also been studied in both dimensions, specifically incommensurate magnetism in $\mathrm{ID}^{77}$ and canted antiferromagnetism in $2 \mathrm{D}^{62}$. Incommensurate magnetism was also found to result from doping antiferromagnetic chains ${ }^{77}$, related to the presence of hidden string order ${ }^{78}$. Although this hidden order is significantly more challenging to directly measure in larger dimensions, in Chapter 4 I discuss our work searching for signatures of hidden order in $2 \mathrm{D}$.

Groups are also actively studying transport in the Hubbard model and exploring cold-atom analogs of well-established condensed matter methods. Spin diffusion in Mott insulators has been examined to show that it is driven by superexchange ${ }^{79}$, and the relationship between charge resistivity and temperature has been found to exhibit a linear dependence, characteristic of bad metals ${ }^{80}$. The Bakr group has recently developed ARPES for cold atoms in the attractive Hubbard model and found evidence for a pseudogap at a temperature $T / t \approx 0.5$, significantly higher than the critical temperature required for superconductivity, $T_{c} / t<0.15^{8 \mathrm{I}}$. This is generally the current status of cold-atom studies of the Hubbard model (other studies exist which have not been mentioned), and the context within which this thesis was written.

Details on our Fermi gas microscope (the "machine") can be found in the many diploma and doctorate theses from our group ${ }^{82,83,84,15,17,21}$. Our current experiment sequence up to lattice load- 


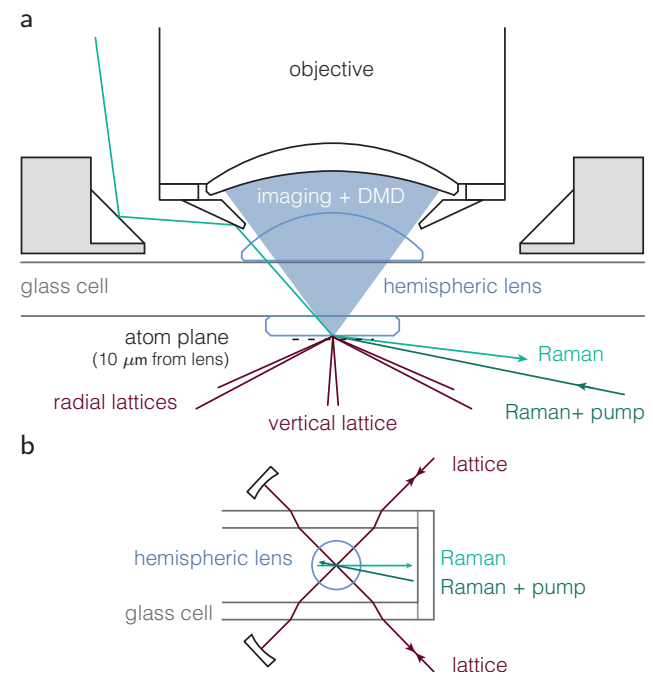

Figure 2.4: Beam geometry of the Fermi gas microscope. a, Side view. Atoms are trapped in a 2D plane of the radial lattices, wavelength $1064 \mathrm{~nm}$. They sit 10 microns from the first element of the high-resolution imaging system, a composite hemispheric lens formed by a superpolished substrate in the glass cell, the wall of the glass cell, and a lens outside of vacuum. Digital micromirror devices (DMDs) in the image plane project optical potentials (650 $\mathrm{nm}$, temporally incoherent) with single-site resolution. During imaging, a vertical lattice $(1064 \mathrm{~nm})$ provides additional vertical confinement. A pair of Raman beams and a pump beam enter the glass cell at an angle chosen to couple to all three lattice directions. b, Bottom view. Radial lattices reflect off of the bottom superpolished and coated surface of the hemispheric lens before retroflecting, giving rise to an additional factor of two in trap depth which is critical for our Raman sideband imaging.

ing is described in detail in Maxwell Parson's thesis ${ }^{17}$. This part of the experimental sequence has remained mostly unchanged for the work presented here. Figure 2.4 depicts the lattice and highresolution imaging beam geometry of our Fermi gas microscope. 


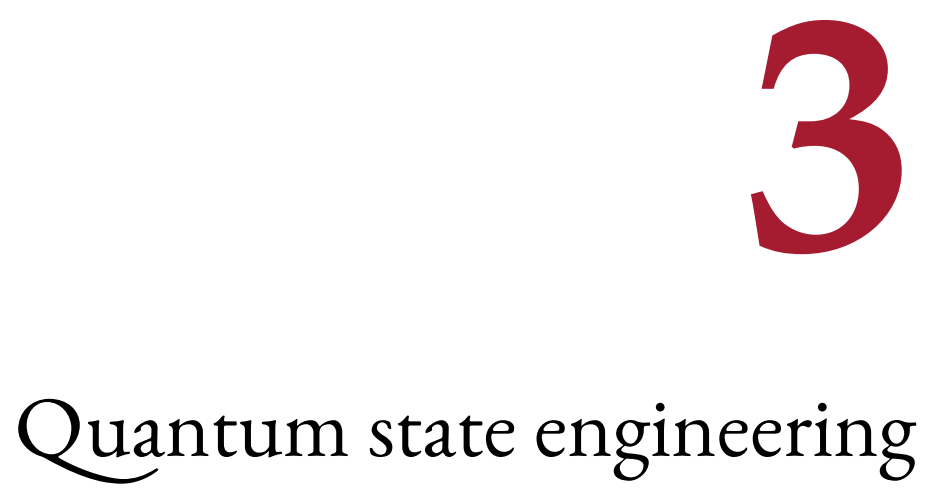

The BIgGeSt CHALlenge, arguably, of quantum simulation with the Hubbard model is realizing the sufficiently low temperatures required. This challenge has motivated our previous work engineering low-noise lattices ${ }^{85,86}$ and developing entropy redistribution techniques ${ }^{20}$, which (among other things) have enabled us to realize our cold-atom antiferromagnet and achieve the coldest tem- 
a

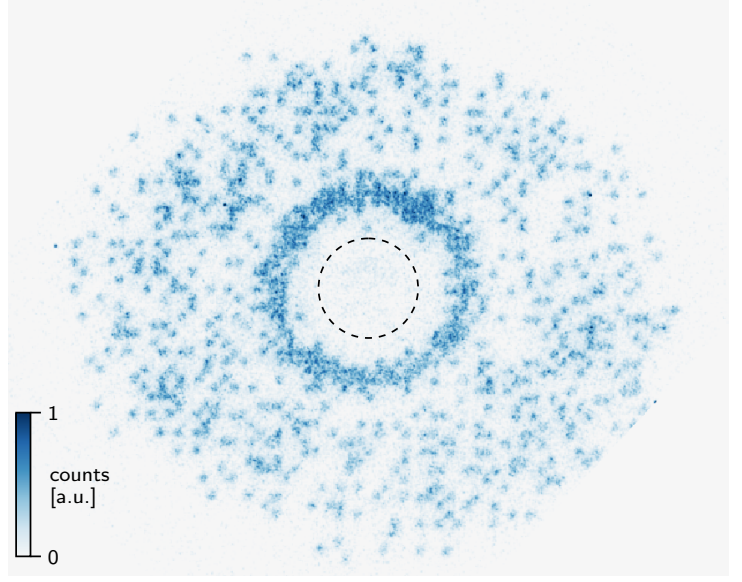

b

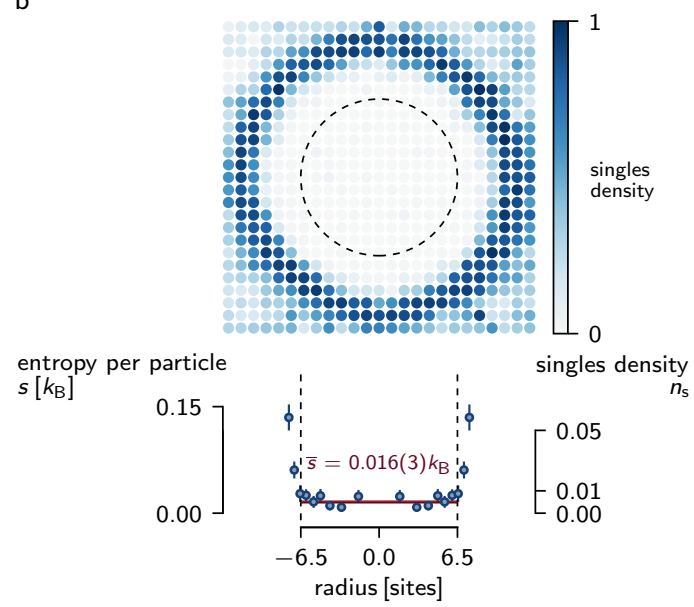

Figure 3.1: Ultra-low-entropy cold-atom band insulator. a, Raw image of atom distribution with an entropy redistribution pattern designed to create a band insulator. The band insulator is in the center of the image, as doubly occupied sites are imaged as empty, with a dilute metal at the exterior. A Mott insulating ring can be seen as the chemical potential increases monotonically from the edge to the middle of the cloud. $\mathbf{b}$, Average singles density in and around the band insulator over 50 images. An azimuthal average confirms that the density is radially uniform and an average over the entire band insulator (disk within dashed circle) yields an average entropy per particle of $\bar{s}=0.016(3) k_{\mathrm{B}}$.

peratures of these systems to-date. At the same time, with these existing techniques we seem to be limited to temperatures of one-quarter of the tunnelling. In this chapter, I discuss our team's implementation of a quantum state engineering (QSE) scheme with the goal of reaching lower tempera-

tures.

\section{I ENTROPY REDISTRIBUTION}

The first indication that QSE was a technique worth pursuing was our realization of an ultra-lowentropy band insulator, which we show in figure 3.I. A band insulator, commonly referred to as an insulator, is a material where the lowest band is completely filled. In our case, because we have two spin species, this occurs when we have two particles per site. Upon imaging these doubly oc- 
cupied sites, atoms undergo light-assisted collisions and the sites are imaged as empty; however, we know these sites are doubly occupied given the trap potential. In fact, with the potential energy shift between the center system and surrounding reservoir, the interaction energy, and an order-ofmagnitude estimate of the temperature, one can use Boltzmann factors to see that for an imaged empty site, the probability of having zero atoms on the site (as opposed to two) is negligibly small.

While single images look quite nice, the band insulator is quite stable as well. By making approximately roo realizations of the band insulator, we find the singles atom distribution to be quite low across the entire system, with an average singles density of $0.004(1)$ within the encircled region. Here and throughout this chapter, all stated uncertainties and error bars denote the standard error of the mean unless otherwise noted. This corresponds to an average entropy per particle of $\bar{s}=0.016(3) k_{\mathrm{B}}$, which we calculate as described in section 3.2.I and estimate to be roughly a factor of 20 lower than that of our coldest cold-atom antiferromagnets. This entropy is also significantly lower than that of the lowest-entropy two-component band insulators realized in cold-atom systems thus far ${ }^{18,87}$.

The first question one might ask in response to these results may be: why is entropy used here, rather than temperature? Indeed, our discussion of Hubbard model physics in Chapter 2 never mentioned entropy, only temperature. Here we discuss the preparation of an cold-atom system, and in this situation, entropy is the natural quantity to use. When loading an atomic cloud from one trap into another, the energies of the quantum states may change and so the temperature may change, but the entropy will stay constant as long as the ramp is adiabatic. More specifically, in our discussion of entropy redistribution the global entropy is assumed to remain invariant while the local en- 
tropy changes; thus a description of the system using entropy is more appropriate. Similarly, when discussing quantum state engineering, the final ramp must be adiabatic; naturally, entropy is more appropriate in assessing adiabaticity. By contrast, when discussing quantum states such as antiferromagnets, the temperature is useful quantity because it can be compared to other energy scales of the system (namely, the tunneling and the superexchange) to gain an intuition for the relevant physics.

Our success in creating the lowest-entropy cold-atom band insulators raises the question: why does entropy distribution work so well for band insulators? We answer this question by explaining how entropy redistribution works. Consider a system $(S)$ and reservoir $(R)$ in thermal equilibrium: $\mathrm{S}$ has very few states below the thermal energy, while $\mathrm{R}$ has comparatively many more. Then a greater number of states are populated within $\mathrm{R}$ relative to $\mathrm{S}$, i.e. $\mathrm{R}$ will have a higher entropy per particle relative to $S$ and acts as an entropy reservoir. If the global entropy per particle is constant, then the entropy per particle within $S$ can be reduced through entropy redistribution and the global temperature can be lowered. Keep in mind that the temperature of the system and reservoir are the same-so in figure 3.I, the central band insulator is just as hot (or cold) as the surrounding metal!

The $2 \mathrm{D}$ Hubbard model contains several phases with different densities of states which can be used for system-reservoir pairs and entropy redistribution. In particular, a coexistence of phases can be realized through inhomogenous particle density in global thermal equilibrium ${ }^{\mathrm{I}}$. A metal exists at low particle density, characterized by a large density of states and high entropy per particle. At half-filling (one particle per site), an antiferromagnet emerges, where spins develop strong anticorrelations with spins on neighboring sites. This phase is gapped in the charge sector by $U$, but has nonzero density of states due to low-energy spin excitations. The band insulator appears when 
the band is completely filled with two particles per site, and thus has a large energy gap equal to the bandgap, vanishing density of states, and vanishing entropy per particle. More specifically, figure 3.2a shows the dependence of entropy per particle on particle density as computed through the numerical linked cluster expansion (NLCE) algorithm ${ }^{88}$, for a homogenous sample with $U / t=8$ and temperature $T / t=1.3$. While our temperatures are significantly lower and we have an inhomogenous sample, the monotonic decrease of entropy per particle with density should persist. Additionally, here there are no long-range correlations, so the local chemical potential changes very little over any correlation lengths in the system. Therefore, the local density approximation is valid ${ }^{89}$ and the local entropy per particle should also be dictated by the local density. Because of the differing density of states, under fixed global atom number and global entropy the density inhomogeneity can be engineered to produce low-entropy states.

We realize the lowest-entropy states by pairing phases with the highest and lowest density of states, i.e. phases which are ungapped and fully gapped. In our case, this is the metal and the band insulator. We create the two subsystems by projecting a spatial pattern of light created with a digital micromirror device (DMDI) onto the atom plane. The pattern is chosen such that in the combined lattice and DMDi potential, there are two spatially homogenous regions with a potential offset of $\Delta \approx 2 U$, thermally connected by a region of intermediate potential offset. When loading into this combined potential, we tune the global chemical potential (by evaporation prior to loading) such that the region of high optical potential supports a metallic state (the reservoir $\mathrm{R}$ ) and the region of low optical potential supports a band insulating state (the system S). Note that the spatial layout of the two regions does not play a role, as long as they are in thermal equilibrium with one another. 
a

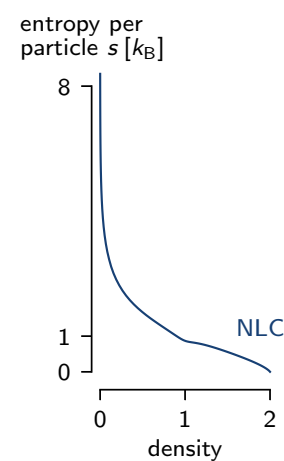

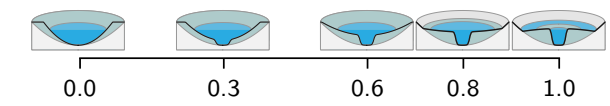

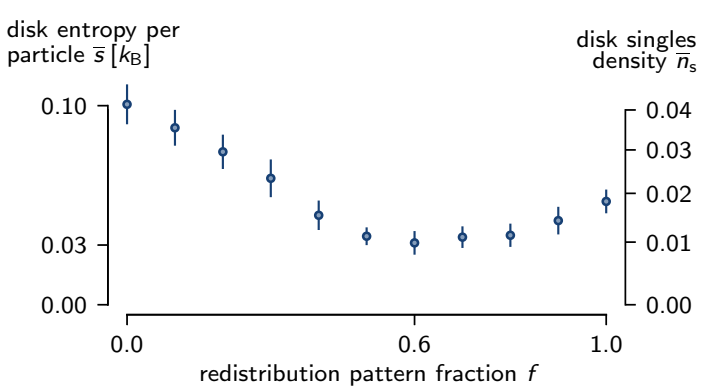

C

nearest-neighbor correlator $\tilde{C}_{s}(1)$

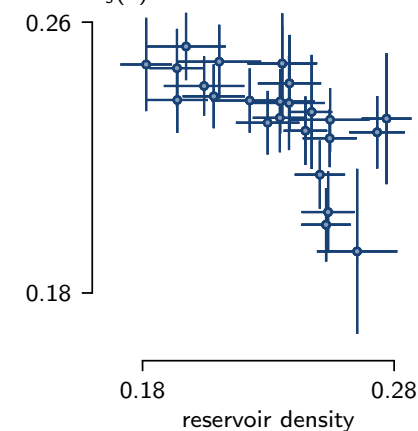

Figure 3.2: Entropy redistribution. a, NLCE numerics for the entropy per particle $s$ in units of $k_{\mathrm{B}}$ for a 2D system at $U / t=8$ and $T / t=1.3$ with homogenous density ${ }^{88}$. The key feature here is the sharp contrast in entropy per particle for densities close to 0 and 2 , which can be used to optimize entropy redistribution. b, Entropy per particle within a 133-site band insulator for patterns ranging between a harmonic trap and a pattern designed for entropy redistribution. While entropy redistribution already occurs in a harmonic trap as a result of density inhomogeneity, under fixed global particle number and global entropy it can be improved by reshaping the potential landscape. The final $f=1$ pattern yields a slightly higher entropy per particle $\bar{s}$ than the optimum, possibly due to the loss of thermal contact between the system and reservoir. c, Sign-corrected nearest-neighbor correlator $\tilde{C}_{s}(1)$ for systems at halffilling as a function of estimated reservoir density. While fluctuations in the reservoir density are large and the size of the reservoir depends strongly on lattice alignment (here a size of 1055 sites is assumed and horizontal error bars denote the standard deviation), there is a clear relationship between lower reservoir densities and stronger nearestneighbor correlations.

Because the local chemical potentials are tuned to create these phases, entropy redistribution for band insulator creation is robust to variations in the tunneling $t$ or on-site interaction $U$. The data presented in figure 3.I has $t / h=0.89(1) \mathrm{kHz}$ in units of the Planck constant $h$ and $U / t=7.7(3)$. However, as long as $S$ has a potential at least $2 U$ deeper than $\mathrm{R}$, the global chemical potential of $\mathrm{R}$ is tuned to create a dilute metal, and the temperature $T$ is much less than $U$, S will contain a band insulator. The limit to creating low-entropy band insulators is not fundamental, but experimental; the absolute laser power available to reshape the overall atom potential is limited ${ }^{21}$. Therefore, some of the data presented in this chapter was taken at $U / t=5.9(2)$, as we were investigating ways to optimize our QSE scheme. For completeness I will specify which parameters were used for each 
result, even though for this study we do not expect a significant change between the two values of $U$ considered.

We also note that by creating an antiferromagnet in $S^{20}$, entropy redistribution does allow us to reach lower temperatures than in a harmonic trap, however it is not as efficient at removing entropy from $S$ because the antiferromagnet can have low-energy spin excitations. Figure $3.2 \mathrm{c}$ shows a scatterplot of several datasets taken with the system at half-filling with varied particle number in the reservoir. We find a general trend between atom density in the reservoir and the strength of the signcorrected nearest-neighbor spin correlator, which may reflect an increase in entropy redistribution efficiency with decreasing reservoir density.

In fact, we can quantify the expected entropy redistribution efficiency. For fixed global entropy $\bar{S}$ and global particle number $\bar{N}$, we can approximate the entropy redistribution efficiency based on the difference in entropy per particle of $\mathrm{A}$ and $\mathrm{B}$ by some factor $\alpha$ :

$$
\left(\frac{\bar{S}}{\bar{N}}\right)_{\text {res }} \equiv \alpha\left(\frac{\bar{S}}{\bar{N}}\right)_{\text {center }}
$$

Then the total entropy per particle is given by the total entropy of the system, divided by the 
total particle number:

$$
\begin{aligned}
\left(\frac{\bar{S}}{\bar{N}}\right)_{\text {total }} & =\frac{\bar{S}_{\text {res }}+\bar{S}_{\text {center }}}{\bar{N}_{\text {res }}+\bar{N}_{\text {center }}} \\
& =\frac{\left(\frac{\bar{S}}{\bar{N}}\right)_{\text {res }} \bar{N}_{\text {res }}+\left(\frac{\bar{S}}{\bar{N}}\right)_{\text {center }} \bar{N}_{\text {center }}}{\bar{N}_{\text {res }}+\bar{N}_{\text {center }}} \\
& =\frac{\alpha\left(\frac{\bar{S}}{\bar{N}}\right)_{\text {center }} \bar{N}_{\text {res }}+\left(\frac{\bar{S}}{\bar{N}}\right)_{\text {center }} \bar{N}_{\text {center }}}{\bar{N}_{\text {res }}+\bar{N}_{\text {center }}} \\
& =\left(\frac{\bar{S}}{\bar{N}}\right)_{\text {center }} \frac{\alpha+\bar{N}_{\text {center }} / \bar{N}_{\text {res }}}{1+\bar{N}_{\text {center }} / \bar{N}_{\text {res }}}
\end{aligned}
$$

Notice that in the limit where the number of atoms in the reservoir is much larger than the number of atoms in the center system, the center entropy is suppressed from the total entropy by a factor of $\alpha$. Given a global entropy per particle in the experiment of approximately $1.0 k_{\mathrm{B}}$, we find a relative entropy reduction of $\alpha \approx 50$ for the entropy redistribution scheme with a band insulator.

Entropy redistribution is already present in most cold atom experiments because they take place in harmonic traps, which create a inhomogenous particle density. By changing the overall potential landscape, while keeping the total atom number, total entropy, and band insulator size constant, we examine how different patterns affect entropy redistribution efficiency. More specifically, we investigate two patterns: the first is a harmonic trap, and the second is the pattern used in figure 3.I with an additional ring of zero particle density between the band insulator and the metal. By linearly interpolating between the amplitudes of the two patterns with parametrization $f$, we see how the corresponding band insulator entropy depends on the potential landscape. The results are shown in figure $3.2 \mathrm{~b}$, with $U / t=5.9(2)$. Entropy redistribution reduces the band insulator 
entropy per particle by more than a factor of 3 compared to the harmonic trap, even for a pattern which has not been optimized for redistribution efficiency as in figure 3.I. We find a slight increase in entropy moving to the final $f=1$ pattern, which may result from a denser reservoir or from a loss of thermal contact between the system and reservoir due to the ring of zero density. This is the pattern which will be used as the initial step of our QSE scheme.

\subsection{Calculations and calibrations}

Before moving on to the details of QSE, here I briefly elaborate on two tools: calculating the entropy from the singles density and calibrating our DMD potentials.

\subsection{ENTROPY CALCULATION}

In the limit of a perfect band insulator, the measured on-site singles density $n_{s}$ is small. We assume that there are no holes and that we have a spin balance. Then on any given site we have a spin up (down) particle with probability $n_{s} / 2\left(n_{s} / 2\right)$, and a doublon with probability $1-n_{s}$. The entropy per particle on a given site $s$ is then given in units of the Boltzmann constant $k_{\mathrm{B}}$ by dividing the total entropy $S$ by the total atom number $N$, of one site across many realizations, all based on the probability $p_{i}$ of having some outcome $i$ and number of particles $n_{i}$ of that outcome:

$$
\begin{aligned}
s & \equiv S / N=\frac{-k_{\mathrm{B}} \sum_{i} p_{i} \ln p_{i}}{\sum_{i} p_{i} n_{i}} \\
& =\frac{1}{2}\left[-n_{s} \ln \left(n_{s} / 2\right)-\left(1-n_{s}\right) \ln \left(1-n_{s}\right)\right] k_{\mathrm{B}}
\end{aligned}
$$


This entropy is an upper bound because correlations between different sites lower the average entropy, but are not included in this estimate. We have also assumed the number of particles per site is exactly 2 , which introduces a maximal error of $0.002 k_{\mathrm{B}}$. This method of calculating the entropy upper bound is used for all entropy values calculated from the data shown in figures 3.I and 3.2. Since the systems that are averaged over in these datasets are homogenous and close to the band-insulating phase, we can calculate $\bar{s}$, the average entropy per particle across the system, by using equation 3.3 but replacing $n_{s}$ with the average singles density across the system $\bar{n}_{s}$.

On the other hand, for the entropy values calculated from data in figures 3.7 through 3.II, the regions that are averaged over include sites that are expected to be empty. These sites have the same relationship between $n_{s}$ and $S$ as sites that are almost full, but the number of particles is now close to o rather than 2. The regions therefore have on average I particle per site since the enclosed regions have the same number of doublon sites as hole sites. We assume an average of exactly i particle per site, which from an inequality in number of doublon sites versus hole sites of up to 5 sites results in a maximal relative error of $2.5 \%$. We calculate the average entropy by measuring $\bar{n}_{s}$ across the entire region, which results in an upper bound for the entropy since entropy is a concave function with respect to singles density. We perform the region averaging to decrease the effect of the bias of the entropy estimator, which decreases as the number of samples increases. For $n \approx 100$ as in the 

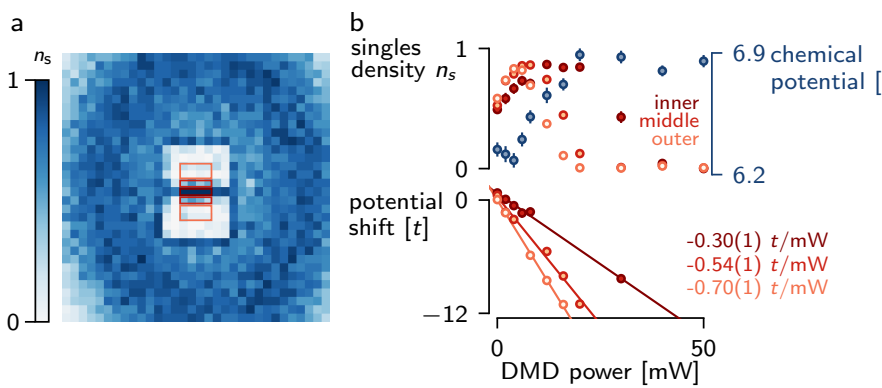

Figure 3.3: Digital micromirror device calibration. a, Density distribution of singly occupied sites with DMD1 calibration pattern and incident optical power of 16 $\mathrm{mW} . \mathbf{b}$, (upper) Singles density of each region highlighted in (a), and global chemical potential, as a function of DMD power. (lower) Potential shift in each region extracted from corresponding singles densities, with fitted line denoting calibration. The potential shift is expressed in units of the tunneling $t$, which is approximately $0.9 \mathrm{kHz}$.

presented data, this bias is maximally $0.006 k_{\mathrm{B}}$. The entropy per particle is therefore calculated as:

$$
\begin{aligned}
\bar{s} & \equiv \bar{S} / \bar{N} \\
& =\left[-\bar{n}_{s} \ln \left(\bar{n}_{s} / 2\right)-\left(1-\bar{n}_{s}\right) \ln \left(1-\bar{n}_{s}\right)\right] k_{\mathrm{B}}
\end{aligned}
$$

Note that this result differs from equation 3.3 by a factor of two due to the difference in particle number.

Uncertainties on entropy per particle values are derived from standard error propagation of the standard deviation of the measured singles density.

\subsubsection{Digital MicromirRor DEVICE POTENTIAL CALIBRATION}

To calibrate the potential created by DMDı, we apply a pattern consisting of two boxes separated by a narrow strip of the same width used in the single-site-wide regions in figure 3.8. The resulting density distribution for an intermediate optical power incident on the DMD ("DMD power") is shown 
in figure 3.3a, with the areas used for calibration indicated by the boxed regions. By changing the optical power incident on the DMD and determining the average density distribution, we determine the effect of the DMD on site occupation, as seen in the upper panel of figure $3.3 \mathrm{~b}$. We also fit the density distributions of the atoms outside of the area affected by the DMD to determine the global chemical potential and temperature.

We then use NLCE calculations to invert the site-dependent singles occupations and extract local chemical potentials ${ }^{88}$. Values above the maximum theoretical singles occupation and within two standard errors of zero density are discarded to limit the domain such that the relationship is oneto-one. From the local chemical potentials, global chemical potentials are subtracted to determine the potential difference caused by the DMD. The differences are then fit to a line whose slope is the potential offset per milliwatt of DMD power, plotted in the lower panel of figure $3.3 \mathrm{~b}$. All data presented in this chapter were taken using the maximum DMD power of $50 \mathrm{~mW}$, where the offset between adjacent sites within and outside the potential (i.e. between the inner and middle regions) is $-2.0(2) U$, and the maximum offset is $-6.0(2) U$, where here $U=5.9(2) t$.

\subsection{Quantum state engineering}

Traditionally, cold atom experiments in optical lattices realize quantum states by loading an evaporatively cooled quantum gas into the lattice potential ${ }^{90}$. This approach has been very successful ${ }^{91, S 1, \varsigma_{2}}$, but the minimum achievable temperatures (for fermionic systems in particular) are limited by reduced cooling efficiency at low temperatures. An alternative approach is QSE. Generally, this 


\section{I. initialization}

schematic

$$
\left.\begin{array}{r}
2 \\
\text { singles } \\
\text { density } \\
0
\end{array}\right]
$$$$
\text { wall - }
$$

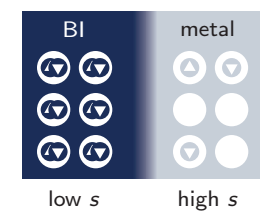

top view

$$
\begin{aligned}
& \left.\begin{array}{r}
\text { singles }^{2} \\
\text { density } \\
0
\end{array}\right] \\
& \text { wall - }
\end{aligned}
$$

3D cutaway

$$
\begin{aligned}
\text { lattice } & \square \\
\text { entropy redistribution (DMD 1) } & \square \\
\text { wall (DMD 2) } & \square \\
\text { combined potential } & - \\
\text { local chemical potential } &
\end{aligned}
$$

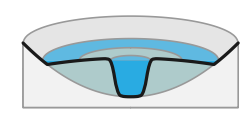

II. isolation
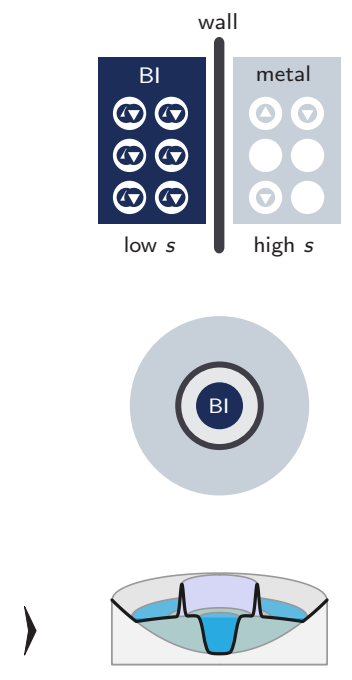

III. adiabatic ramp

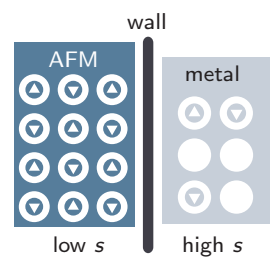

Figure 3.4: A quantum state engineering protocol. The three steps of the QSE protocol are depicted in increasing levels of specificity. The schematic shows how we use a coexistence of metallic and band insulating (BI) phases and controlled separation to create a low-entropy antiferromagnet (AFM). The top view shows the physical geometry used in our experiment, however the success of this protocol should be robust to the exact arrangement of the system and reservoir, as long as they can be thermally connected and disconnected. The 3D cutaway shows how we control optical potentials to implement each step of the protocol and move from one step to the next.

method realizes an isolated pure quantum state by initializing one wavefunction under an initial Hamiltonian, then changing the Hamiltonian while preserving coherence during time evolution so that the accompanying wavefunction becomes the target state. Several platforms have used different versions of QSE to create desired quantum states ${ }^{92,93,94,95,96}$, and schemes have been proposed for ultracold fermionic atoms ${ }^{97,98,99}$. The site-resolved readout and control afforded by quantum gas microscope experiments $^{100,47,33,87,20,78,35,59,60,62}$ are perfect tools to implement QSE of many-body states of ultracold fermionic atoms in optical lattices.

We design a protocol as outlined in figure 3.4 consisting of three steps. In step I, the ultra-lowentropy band insulator is initialized through entropy redistribution and is in thermal contact with a 
metal. In step II, the band insulator is thermodynamically isolated from the metallic entropy reservoir. Both regions maintain the same entropy per particle as in the previous step. In step III, the Hamiltonian is adiabatically modified such that the band insulator becomes an antiferromagnet. Crucially, if the ramp is truly adiabatic, then the final state will exhibit the same ultra-low entropy per particle as in the band insulator.

The protocol is implemented by varying the laser power on two digital micromirror devices. Step I uses DMDi as described in the previous section. The entropy redistribution potential contains a ring of zero particle density between the band insulator and metal, equal to the $f=1$ pattern of figure 3.2. For step II, a second digital micromirror device ( $\left.\mathrm{DMD}_{2}\right)$ is used to project a ring-shaped potential wall between the band insulator and metal. The wall is located within the ring of zero particle density to avoid disturbing the atom distribution and increasing the band insulator entropy. Some empty sites are included within the wall, as seen in the "top view" and " $3 \mathrm{D}$ cutaway" of figure 3.4. Finally, for step III we keep the $\mathrm{DMD}_{2}$ laser power constant, but ramp off the DMDi laser power. The resulting potential offset $\Delta$ almost vanishes, leaving a weak harmonic confinement within the central system from the lattice lasers.

Notice that in our depicted protocol, we have circular boundaries on our band-insulating and empty regions. This is robust to alignment because there are no straight edges to align with the rows and columns of the square lattice. At the same time, this necessarily results in some sites with intermediate particle numbers, simply because the circle pattern illuminates some sites on its edge with less light than is required to shift the potential by the full $2 U$. This increases the entropy per particle of our system because here the system is defined not only as the band insulator, but as the entire 
DMD 1

top view

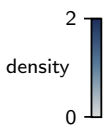

DMD 2

top view

combined

3D cutaway

experimental

average
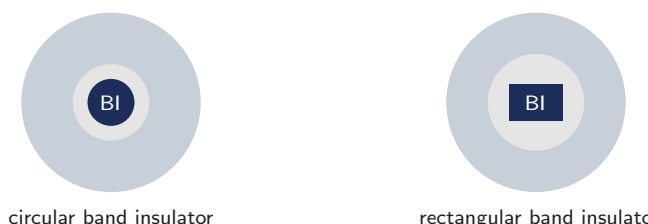

rectangular band insulator

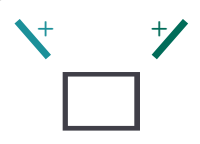

striped band insulator circular band insulator

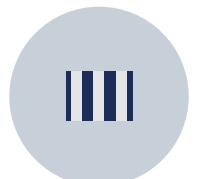

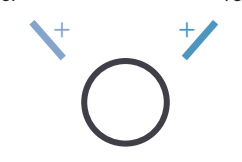

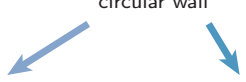

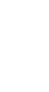


with empty sites and create a system with a known fixed particle number. However, as can be seen in the map of average singles density, if the edges of the rectangle are not aligned perfectly to the square lattice, we create sites with intermediate density and increase the entropy of our system. One of the most stringent configurations requires control over both the number of atoms in the band insulator and the number of empty sites. For example, we can create a striped pattern of alternating columns of doublons and holes, such that the numbers of each are equal. In this case, the inner edge of the isolating wall must also take the shape of a rectangle and must directly border the striped pattern of DMDi. Here we require perfect alignment of both DMDs. We can slightly ease some of the alignment constraints for this pattern by creating stripes of width 2 sites rather than I site, while maintaining other attributes such as the minimum level of particle transport required to reach one particle per site. An additional increase of control would be to ensure that the final potential of the QSE protocol is homogenous across the system by flattening it with one of the DMDs; however, this requires full control of the particle number which we do not currently have given the associated sensitivity to alignment.

\subsection{EXPERIMENTAL IMPLEMENTATION}

Our experimental sequence has remained largely unchanged since our realization of a fermionic Mott insulator ${ }^{18}$. The exception is lattice loading, which we modify with the addition of DMDi for entropy redistribution ${ }^{20}$ as seen in figure 3.6a. In each sequence, the pattern displayed on DMDI remains fixed, while the laser power of the incident beam is ramped up to image the pattern onto the 

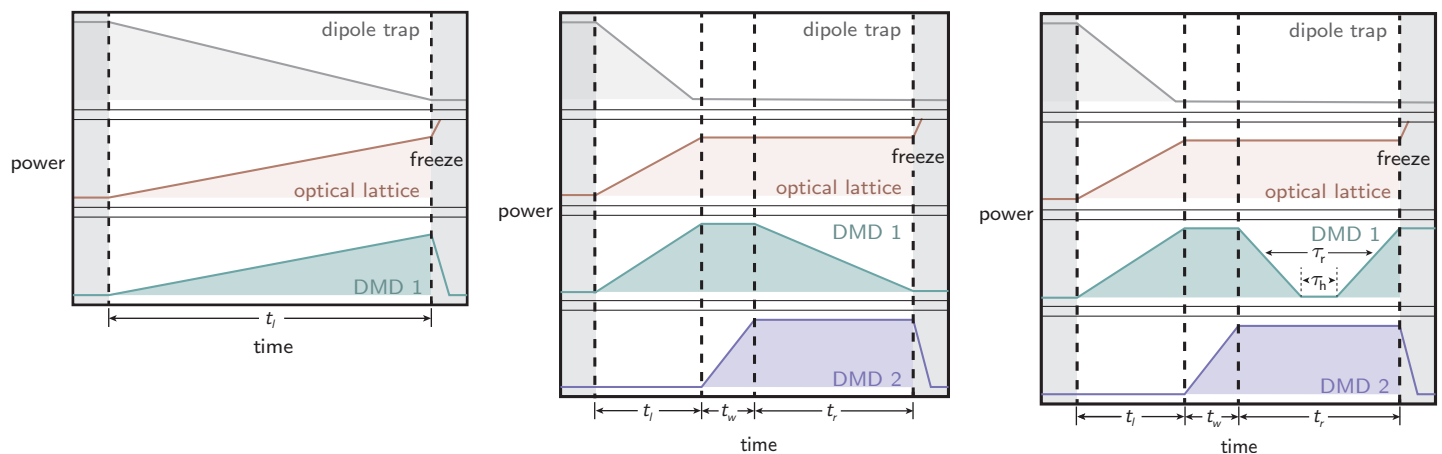

Figure 3.6: Optical power ramp protocols. a, Ramp protocol for lattice loading with entropy redistribution, where atoms are handed off from a crossed dipole trap into the combined optical lattice and DMD1 potential. The lattice is then immediately and quickly ramped up to freeze out the tunneling in preparation for imaging. $\mathbf{b}$, Ramp protocol for quantum state engineering. After loading into the lattice and DMD1 potential, the DMD2 optical power is ramped up to isolate the system and reservoir, after which the potential offset between doublons and holes introduced by DMD1 is ramped off. The lattice freeze follows. c, Ramp protocol for the round-trip measurement used to characterize the quantum state engineering scheme. All quantum state engineering ramps are reversed to recover the original isolatedsystem configuration. Furthermore, the extent and duration of the final ramp can be varied, as well as a hold time before ramp reversal, to characterize the ramp adiabaticity and heating.

atom cloud. We load into the combined lattice and DMDi potential simultaneously.

The QSE scheme expands upon this sequence and is shown in figure 3.6b. During the entire sequence, the patterns on DMDi and 2 are kept static, while the power of the respective incident beams are ramped up and down to implement each step of the protocol. The lattices are then ramped up quickly to 'freeze' out all physics for optional spin removal and imaging. We now characterize each step of the QSE protocol.

\subsection{INITIALIZATION}

Because imaging does not distinguish doublons from holes, we must explicitly check that doublons are loaded into the desired regions of our initial configuration. We determine the minimum re- 

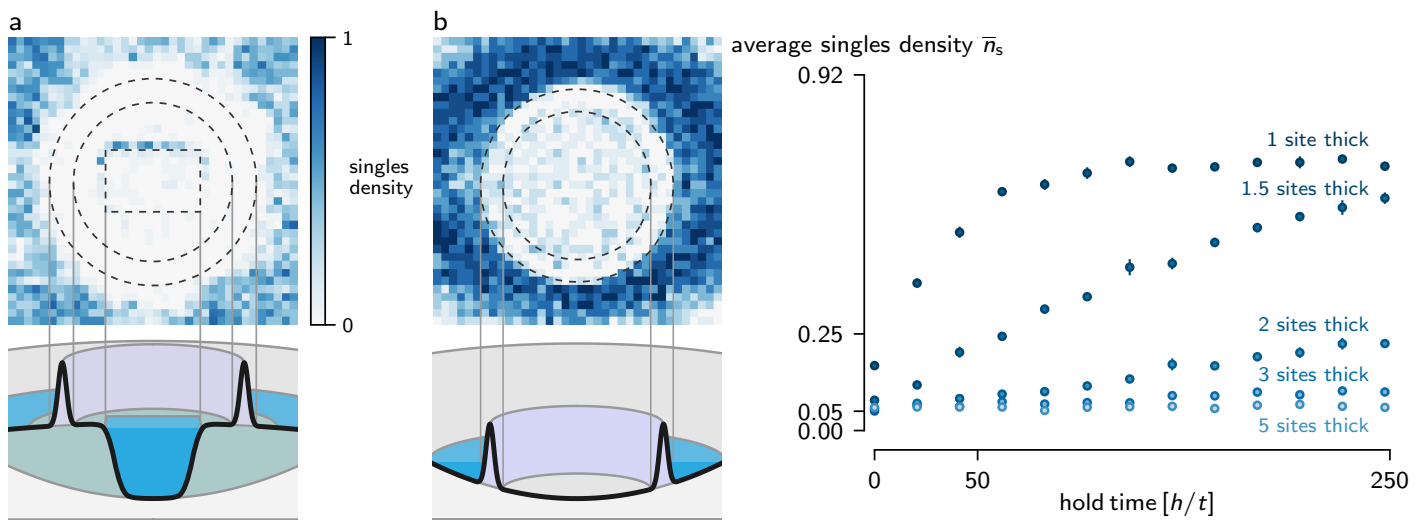

Figure 3.7: Characterization of isolation in QSE. a, Density distribution of singly occupied sites (40 realizations) and configuration of entropy redistribution at $U / t=5.9(2)$ after isolation. The isolated system consists of an approximately equal number of doubly occupied and unoccupied sites, whose boundary is denoted by the dashed rectangle. Imperfections in the optical potential manifest as singly occupied sites, as seen at the upper edge of the box. Dashed circles denote the boundaries of the isolating wall, which do not significantly modify the atom distribution from entropy redistribution alone. b, Verifying wall insulation. After preparing a region containing fewer than 3 atoms, we raise up a circular wall as in the quantum state engineering protocol. The potential used to prepare the initial state is ramped off, leaving an atom distribution (upper left) and attractive harmonic well potential (lower left). The rate of particle transport into the central region is measured, for varying wall thicknesses in lattice sites (right). For our QSE protocol, we use a wall thickness of 3 lattice sites.

quired potential offset by incrementally increasing the offset from zero and observing the associated atom density of the box. As this offset is increased, the density increases past half-filling and into the band-insulating regime. This indicates the minimum potential offset we must have to create sites with doublons neighbored by sites with holes.

For the results presented here, DMD patterns are aligned by hand by changing the patterns and measuring the resulting atom distribution; however, we now automatically track and compensate for sub-lattice-site drifts of the DMD with respect to the lattice phase. 


\subsubsection{IsOLATION}

In figure $3.7 \mathrm{a}$, I show the average singles density over 40 realizations of an 8 -site by 12 -site rectangular band insulator with a circular wall, at $U / t=5.9(2)$. The wall has a thickness of approximately 3 sites, and the system is defined to be the region inside the wall, which contains both empty and doubly occupied sites. Here the system entropy per particle is $0.25(1) k_{\mathrm{B}}$. This entropy is greater than that of the pure band insulator because it includes both doublon and hole regions; indeed, the

pure band insulator entropy per particle away from the box edge is only $0.08(1) k_{\mathrm{B}}$, so the greatest entropy contribution to the system is from the boundary between the regions. The ring-shaped wall has a negligible effect on initial entropy, confirmed through comparing the entropy with and without the wall.

We independently ensure that the wall isolates the central system from its reservoir. To do this, we study particle flow into a system designed to contain only empty sites. The entropy redistribution pattern is modified to be completely deconfining in the center, such that the wall encloses a system with fewer than 3 particles. The outer radius of the wall is kept constant at $\mathrm{I} 2$ sites. Upon removal of the deconfining potential, the center becomes an attractive harmonic well as depicted in figure $3.7 \mathrm{~b}$. If the wall is not insulating, particles can tunnel into the system. We plot the singles density over time, for various thicknesses of the confining wall.

As expected, the rate of inward particle flow decreases with wall thickness. As several virtual tunneling processes to high energy states are required for a particle to tunnel across the wall, we expect the effective wall tunneling time to grow exponentially with the wall thickness. Experimentally, 
however, the wall cannot be made arbitrarily wide, because it must occupy a region containing no particles. Increasing the size of this region decreases the size of the usable system. We note instead that the wall need only be sufficiently insulating on the timescale of the adiabatic ramp into the final many-body state. Therefore, we find the minimum wall thickness for which on average the entropy increases by less than about $0.05 k_{\mathrm{B}}$, given a conservative ramp time of $40 \mathrm{~ms}$. This corresponds to a wall 3 sites wide.

It is admittedly more challenging to calibrate the potential offset due to the optical potential from $\mathrm{DMD}_{2}$ than it is for DMDi. There are several reasons for this. First, the beam waist of the laser incident on $\mathrm{DMD}_{2}$ is much smaller than that on $\mathrm{DMD}$, which creates a strong variation in spatial intensity and therefore a locally changing offset independent of the DMD pattern. Second, when the wall pattern is set to a circular ring, it is not commensurate with the rectangular array of DMD pixels and creates a wall with smooth edges. Finally, we use spatially incoherent light for projecting the DMD pattern, so the actual light intensity seen by the atoms depends nonlinearly on the fraction of mirrors that are turned on in a given spatial region of the DMD. This necessitates a separate calibration for each DMD pattern. This was done for DMDi because the calibration is quantitatively important to our analysis; however this is not the case for $\mathrm{DMD}_{2}$.

Nevertheless, we can create a simple model for the suppressed tunneling across the wall by considering a ID lattice potential with $L$ sites offset by $\Delta$. The tunneling across this $L$-site wall is then suppressed from the bare tunneling $t$ by $(t / \Delta)^{L}$, as it requires $L$ virtual tunneling processes to sites which are detuned in energy by $\Delta$. From this model and using the short-time dynamics for the I-site wall, we determine a wall height estimate of $\Delta=80 t$. This estimate is roughly consistent with 
the observed timescales for thicker walls and the DMDi calibration adjusting for differences in the maximum optical power and beam waist.

\subsubsection{Adiabaticity}

The final step in our quantum state engineering scheme is to convert the initial state into the target many-body state. For this measurement we use the disk pattern for the band insulator to reduce alignment sensitivity. We also set $U / t=7.7(3)$ so that we can compare our correlation strength to previous measurements and fit to NLCE numerics. To ensure half filling in the final state, the wall diameter is set such that the numbers of holes and doublons within the system are approximately equal. After initialization and isolation, we slowly remove the potential offset between holes and doublons by reducing the DMDi laser power. In figure 3.8a, we show the measured singles density $n_{s}$ after a $40 \mathrm{~ms}$ linear ramp of the potential offset. The atomic density extends over the entire system and sharply decreases at the inner edge of the wall, indicating particle transport has occurred from the doublon core to the surrounding empty sites. The inner and outer regions are separated by the insulating wall, marked by a ring of empty sites. In the final state, atoms in the system are expected to show antiferromagnetic correlations, whose strength reflect the adiabaticity of the ramp. The nearest-neighbor spin correlations are strongly antiferromagnetic with values up to $C_{s}(d=1)=-0.21(1)$. These observations demonstrate a successful implementation of quantum state engineering, where a strongly correlated many-body state is created from an initially uncorrelated band insulator of doublons.

Locally changing density and spin correlations within the system originate from the underlying 

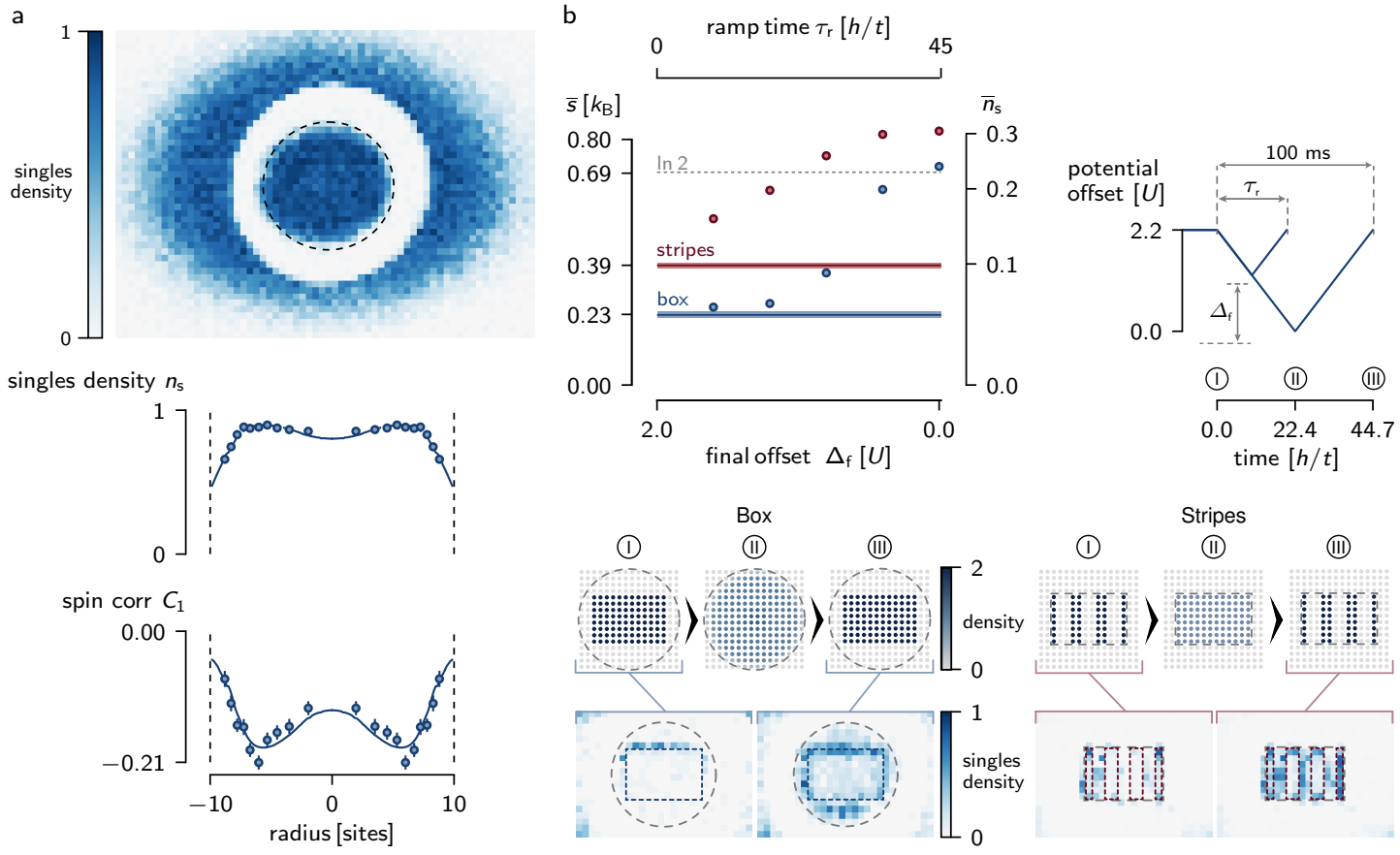

Figure 3.8: Characterization of final state from QSE. a, Average density map of 41 images after ramp with initialization via disk pattern (upper), along with corresponding density and nearest-neighbor spin correlator profiles versus radius after ramp (lower). The nearest-neighbor correlations are antiferromagnetic with a strength of up to $C_{s}(1)=-0.21(1)$. A simultaneous fit to both profiles (solid line) gives a temperature of $k_{\mathrm{B}} T / t=0.46(2)$. The fit is limited to radius 9, to avoid effects from the insulating wall. Error bars denote standard error of over 40 sets of correlation maps and azimuthal averaging. Here $U / t=7.7(3)$. b, Examining final ramp adiabaticity at $U / t=5.9(3)$. A round trip measurement beginning with an isolated box of doublons surrounded by holes demonstrates non-adiabaticity predominantly in the second half of the offset ramp-off (upper left). Adiabaticity is not significantly improved by initializing the holes and doublons in stripes. Horizontal lines with shading indicate reference measurements and uncertainty, taken with no ramp. Lower panels show schematic images for the particle density $n$ and measured average singles density maps for the box (left) and stripe (right) configurations at different times throughout the round-trip ramp. Dashed lines indicate the wall inner edge, while dotted lines enclose band insulating regions. Error bars are smaller than the markers, and denote standard error of 40 (187) measurements for the box (stripe) pattern. 
harmonic confinement created by the lattice lasers. As seen in figure $3.8 \mathrm{a}$, the maximum in the singles density radial profile indicates the density is above half-filling in the center and continuously decreases for larger radii. We intentionally keep this confinement to study whether the system is in thermal equilibrium. When applying a simultaneous fit of exact theoretical predictions to both measured radial profiles with shared fit parameters, we find reasonable agreement ${ }^{88,19}$. This shows that the system is consistent with a state in thermal equilibrium. In order to determine whether deviations from thermal equilibrium or finite-size effects are present, more detailed knowledge of corrections to the exact confinement potential is required.

From the fit we obtain a temperature of $k_{\mathrm{B}} T / t=0.46(2)$, which is comparable to the temperatures achieved so far in harmonic traps ${ }^{62}$, but still higher than the lowest value of $k_{\mathrm{B}} T / t=0.25(2)$ achieved with entropy redistribution ${ }^{20}$. Although this temperature is surprisingly low given the simple ramp scheme used here, the system is still far from the ground state. Besides the nonzero entropy of the initial band insulator, this nonzero temperature may result from non-adiabaticity of the ramp or residual heating. We now explore both possibilities.

We first study non-adiabaticity by examining the entropy increase after completing and reversing the offset ramp as depicted in figures $3.6 \mathrm{c}$ and $3.8 \mathrm{~b}$. For this measurement we use the box pattern for the lowest initial system entropy. As heating effects are negligible in the initial state, a perfectly adiabatic process implies measuring the same entropy as this initial state. When varying the endpoint of the ramp $\Delta_{f}$, we find that the entropy per particle increases steadily as the ramp endpoint decreases as shown in figure $3.8 \mathrm{~b}$. The qualitative shape of the curve suggests a lack of adiabaticity largely throughout the second half of the ramp. In this regime, $\Delta \approx U$ and particles can freely tun- 
nel out of the doublon core. For the full two-way ramp, we find an entropy increase of $0.46(2) k_{\mathrm{B}}$. Although this increase strongly indicates a non-adiabatic ramp, it may actually be caused by greater heating rates during the ramp, for example due to changes in the many-body energy spectrum.

We repeat the adiabaticity measurement for an initial system consisting of alternating stripes of holes and doublons surrounded by a box-shaped wall, which is depicted in the bottom right of figure $3.8 \mathrm{~b}$. While the initial entropy is worse than that of the box due to the more complex potential landscape, crucially this configuration yields no significant improvement in entropy increase. This suggests that the dominant reason for non-adiabaticity lies within the many-body physics occurring during the ramp, which strongly depends on how the ramp is implemented and what intermediate phases are crossed ${ }^{\mathrm{IOI}, \mathrm{IO} 2, \mathrm{IO} 3}$. An improvement could be to avoid a closing charge gap in the manybody spectrum during the ramp, possibly by using a double-well superlattice. Such a configuration has been predicted to be very efficient in numerical simulations ${ }^{99,104}$.

\subsubsection{HEATING}

To distinguish heating during the ramp from non-adiabaticity, we measure the heating rate for each ramp endpoint by holding for a variable time $\tau_{h}$ before reversing the ramp and measuring the resulting entropy as depicted in figure 3.9a. As seen in b and $c$ of the same figure, heating rates are generally greater than the initial heating rate, with values up to $2.1(2) k_{\mathrm{B}} / \mathrm{s}$. The observed increase in heating rate at $\Delta \approx U$ indicates a drastic change in the many-body energy spectrum, as already suggested by the non-adiabaticity measurement of figure $3.8 \mathrm{~b}$. From the measured rates, we estimate an entropy increase from heating of $0.06 k_{\mathrm{B}}$ for the full ramp. This indicates that the majority of 

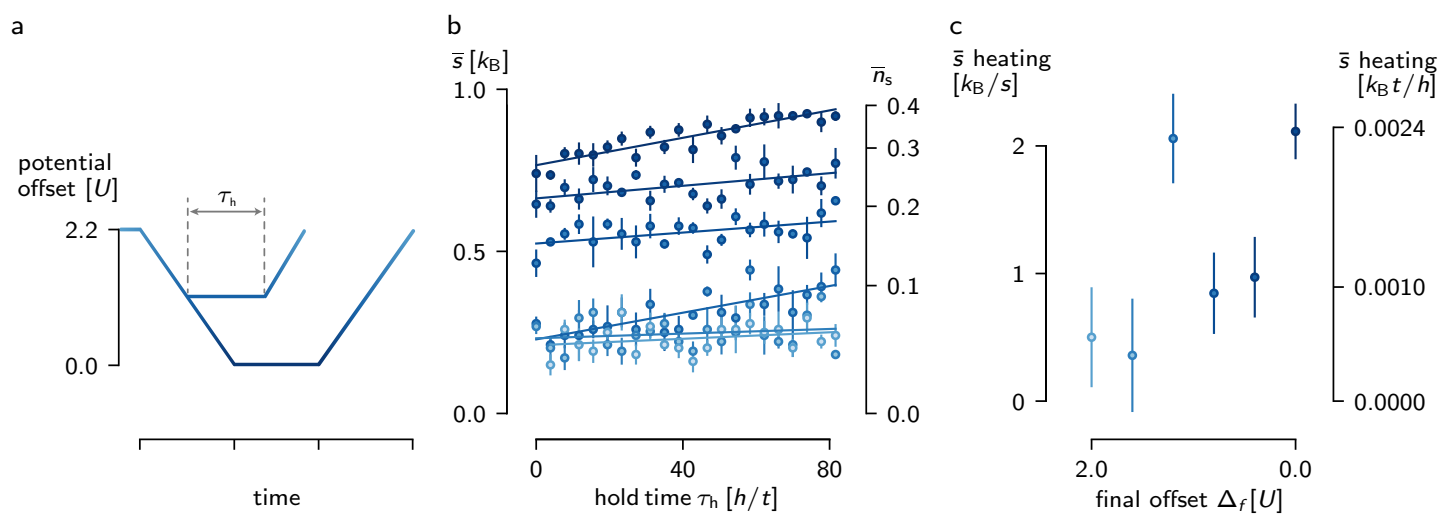

Figure 3.9: Heating during QSE. a, Ramp used for potential offset between the doubly occupied and unoccupied sites. The offset is decreased to some value, at which point the sample is held for some time to measure a heating rate. All measurements are taken after recovering the initial configuration for consistency, and offset ramps are set to the same duration. b, Measured entropy per particle as a function of hold time $\tau_{h}$ for various potential offsets. Linear fits are used to quantify heating rates. Error bars for entropy measurements (right) denote standard error of 5 measurements. c, Extracted heating rates at various points throughout the ramp. Error bars are from the fits in (b).

entropy increase does not originate from heating, but rather from non-adiabaticity. However, when decreasing the ramp rate for the full two-way ramp with zero hold time, as depicted in figure $3.8 \mathrm{~b}$, the final entropy increases. This indicates that any improvement in adiabaticity is insufficient to overcome heating during the additional ramp time.

\subsection{Future strategies}

While this QSE protocol worked surprisingly well given its simplicity, I cannot help but feel slight disappointment that we were not able to immediately improve it. With this protocol we can easily adjust the initial balance of doubly occupied and unoccupied sites to vary the doping of the sample on the single-atom level, although it is not clear how the adiabaticity requirements will vary with doping. If our final ramp were more adiabatic, then we would have a highly promising pathway 


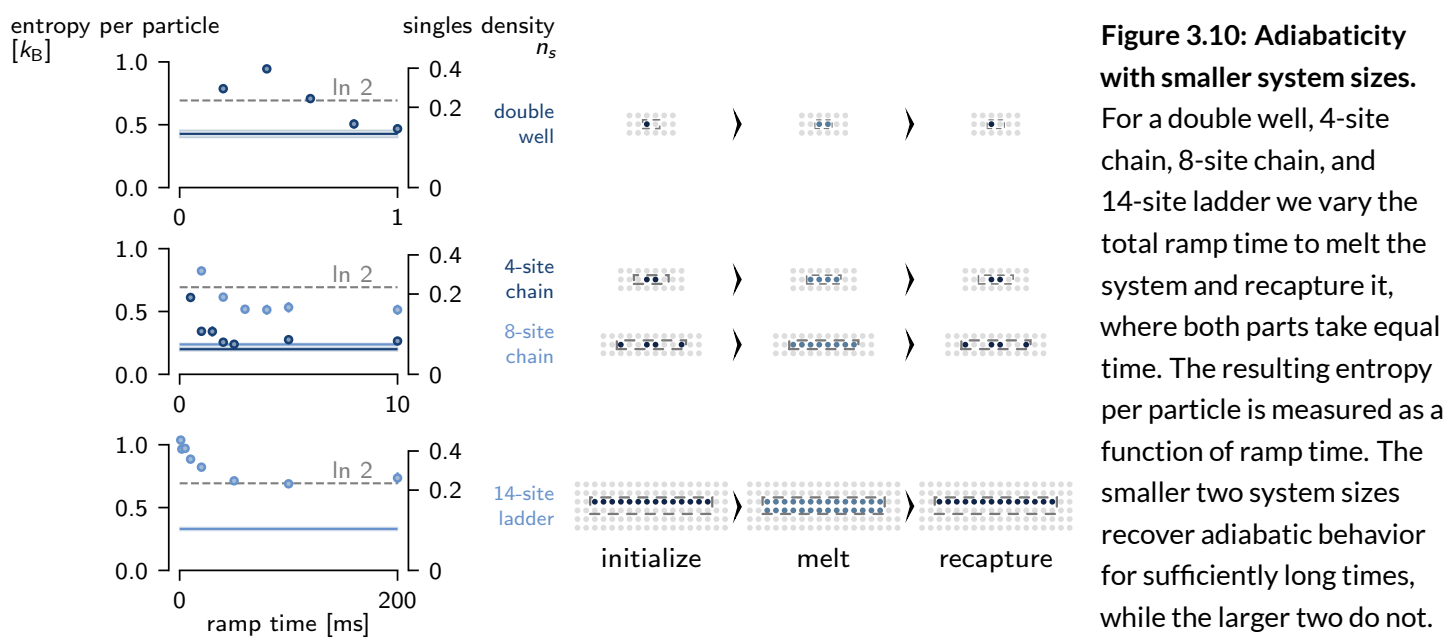

towards investigating low temperature doped states of the Hubbard model and searching for signatures of a $d$-wave superfluid state ${ }^{\text {Ios }}$.

Along the way, we studied smaller system sizes as well as an isolated double well configuration with two adiabatic ramps designed to target the charge and spin sectors of the Hubbard model separately. I will discuss both studies as a segue to my outlook on QSE for reaching ultra-low entropy states of the Hubbard model.

\subsection{SMALler SYSTEM SIZES}

Our quantum state engineering protocol is limited by the non-adiabaticity of the ramp into the many-body state. Provided that the energy gap scales with the system size, we briefly investigate smaller system sizes with regards to successful adiabatic state preparation. For a double well, 4 -site chain, 8-site chain, and I4-site ladder, after initializing with half of the sites empty and half doubly occupied, we isolate the system and completely ramp off the potential offset between the empty and 
doubly occupied sites. We then reverse the ramp. The time to ramp off the potential offset and melt the system is set to be equal to the recapture time, and this total time is varied. While the ramp is not expected to be adiabatic at short times, if it is adiabatic at long times then the entropy per particle of the recaptured state should equal the entropy per particle prior to melting. The results of entropy per particle as a function of total ramp time are plotted in figure 3.Io.

Notably, for both the double well and 4-site chain, there exist sufficiently long ramp times where the final entropy per particle approaches that after initial preparation. In the 8-site chain and I4-site ladder systems, there is a noticeable decrease in entropy per particle as the ramp time is increased. However, for ramp times longer than approximately 3 and $50 \mathrm{~ms}$, respectively, the entropy per particle seems to remain constant at a level significantly above the preparation value. In particular, the entropy per particle in the I4-site ladder does not decrease beyond $\ln (2) k_{\mathrm{B}}$, however we have not investigated if this is physically insightful or simply a coincidence.

It is certainly surprising that we were not able to achieve full adiabaticity with the 8-site chain. Exact diagonalization of the 8-site chain indicates a gap of $0.2 t$, compared to a $0.3 t$ gap for the 4 -site chain (recall, in our experiments $t \approx 1 \mathrm{kHz}$ ). This suggests that technical factors may be responsible. For one thing, it is not clear what the effect is of having such steep changes in the potential. In particular, if the position of the DMD pattern fluctuates relative to the lattice, this shaking can cause heating which is dependent on the size of the boundary relative to the system size. One could test this by examining fixed system sizes of variable aspect ratio. Beyond the question of adiabaticity, our preparation fidelities can also be improved, and may also have been limited by our ability to align the DMD pattern to the lattice. 


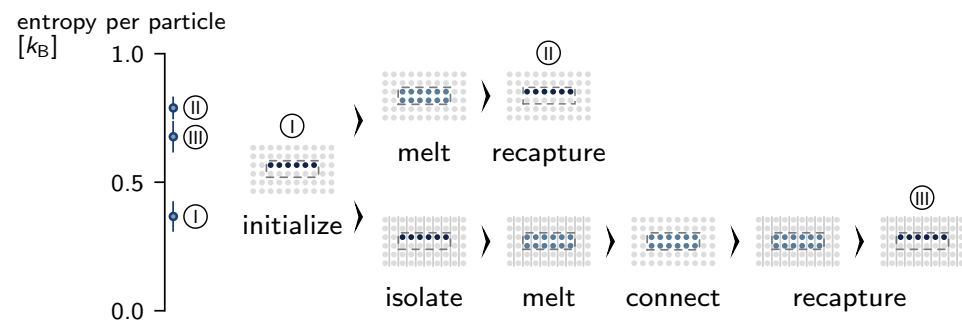

Figure 3.11: Investigating more sophisticated QSE protocols. We test a protocol where pairs of sites each with one doublon and one hole are isolated and individually melted, then connected to other such pairs. After recapturing into the initial configuration, the entropy per particle is measured. Results are similar to the original protocol but may show slight improvement.

\subsubsection{ISOLATED DOUBLE WELLS}

Intuitively, one might think to improve upon the quantum state engineering protocol by separately ramping in the charge and spin sectors in the Hubbard model. More specifically, all of the experiments mentioned thus far attempt to change the charge distribution and the spin distribution concurrently. Alternatively, it is possible to first change the charge distribution by turning each doublon on one site into a singlet on two sites, so that there is one particle per site. The spin distribution can then be changed by introducing tunnelling between singlets.

We perform a preliminary study of this more sophisticated scheme as depicted in figure 3.II. This begins with an isolated 6-site ladder with doublons along one leg of the ladder and empty sites along the other, much like the I4-site ladder system in figure 3.Io. Our original QSE protocol would be to melt the system and remove the potential offset between the two legs, then restore the offset to examine the resulting round-trip entropy. By contrast, this scheme begins by isolating each rung of the ladder (i.e. double well) from its neighboring rungs through increasing the trap depth and consequently reducing the tunneling in that direction. Next, the potential offset between doublon 
and hole is removed, allowing tunneling along each rung which is about an order of magnitude larger than that across rungs. In the adiabatic case this melts the doublon and hole into a singlet. The trap depth of the lattice along the ladder legs is then reduced to connect the double wells and allow isotropic tunneling. At this point if all steps are adiabatic we have an ultra-low entropy manybody state. We then reverse all ramps to recapture the initial configuration, and measure the particle distribution to calculate the entropy per particle.

With about a week's worth of work, most of which went into alignment, we were able to see a slight indication that this more sophisticated scheme might be preferable. Figure 3.II shows the entropy per particle of the initial isolated configuration, as well as that of the recaptured configuration for both the initial and more sophisticated protocols. While the entropy gain is still comparable for both techniques, there are known contributions to the entropy gain for the more sophisticated scheme which are purely technical and can be resolved. In particular, when the ladder rungs are isolated through increasing the trap depth, the overall harmonic confinement increases as well. The resulting increase in chemical potential may be contributing to some of the measured entropy increase. By compensating for this additional harmonic confinement or implementing a similar scheme without this effect, it may be possible to achieve lower entropies and perhaps even adiabaticity.

\subsection{OutLook}

Several improvements are underway on the experiment based on what we have learned in these studies. First, we have implemented a position calibration for both DMDs to adjust for slow drifts in 
their alignment relative to the lattice sites. This has enabled us to initialize desired states much more easily. More importantly, it allows us to take large datasets without drifts in our initial preparation entropy caused by DMD misalignment.

Second, we are designing and testing a superlattice setup, versions of which have been implemented before with high success ${ }^{106}$, to implement a quantum state engineering protocol where doubly-occupied sites are split into two sites. The idea is to add interfering lattices to the existing (non-interfering) lattice geometry. By doing so one can initialize in a larger-spacing square lattice with doublons on every site, continuously split each site into a double well while maintaining suppressed tunneling to the other double wells, and then continuously connect to a square lattice geometry with an average of one particle per site. Crucially, this scheme will not have the strong sensitivities to alignment or possible heating from our DMD. I very much look forward to the installation of this upgrade into our experiment and the studies to follow. 


\section{4}

\section{String patterns}

IN OUR FIRST STUDY OF MAGNETIC CORRELATIONS ${ }^{19}$, we found something we did not fully understand: a sign change in the diagonal next-nearest-neighbor correlator at a doping of approximately o.I5. The Zwierlein group saw the same effect at a higher temperature of $T / t \approx 1.2$ and a doping of $0.2 \mathrm{I}$ (singles density of 0.62 ), accompanied by a sign change in the moment correlator, 
and pointed out that it was similar to Pauli suppression as seen with non-interacting fermions ${ }^{107}$. Although this may have been the case, given that our temperatures were on the order of the superexchange energy, $T / t=0.54(7)$, it was possible that something else was going on that was intimately related to open questions of how holes behave in an antiferromagnet. As we reached even lower temperatures of $T / t=0.25(2)$ and realized an full-fledged antiferromagnet, we found that this effect persisted $^{20}$. The introduction of holes into the antiferromagnet didn't simply destroy antiferromagnetic order, as suggested by most spin observables which seemed to monotonically approach zero upon doping. Rather, the diagonal next-nearest-neighbor spin correlator crossed zero. And this is how we, with the help of theory collaborators Fabian Grusdt, Annabelle Bohrdt, Michael Knap, and Eugene Demler, started thinking about microscopic models for the doped Hubbard model.

I see this chapter as posing and beginning to address two key questions. First, how can we extract additional useful information in our site-resolved snapshots, beyond what is contained by a twoor multi-point correlation function? Second, in our experimental parameter regime, can we already begin to make distinctions between candidate microscopic theories for the doped Hubbard model? In the spirit of these two questions, I will begin the chapter by providing a working understanding of the theories necessary to understand and contextualize the experimental work, followed by a description of the experimental dataset. Next I will spend the bulk of the chapter introducing the new observables we created to characterize doped antiferromagnets, and showing how we used them to evaluate candidates for microscopic theories of the doped Hubbard model. To assess the usefulness of our new observables, I will also show the conventional spin observables of two-point spin correlation functions and staggered magnetization for experiment and theory. The last measurement of 
this chapter extends beyond our two key questions; we examine the charge correlations of the doped Hubbard model to serve as a benchmark for past, present, and future theoretical work. The chapter concludes with a brief presentation of ongoing and future research directions.

\section{I Theoretical BaCKground}

A wide array of theoretical techniques have been applied to the doped Hubbard model, including phenomenology, numerics, mean-field theories, and microscopic theories. Phenomenological approaches tend to focus on specific observed effects and therefore individual phases of the phase diagram, such as stripe phases in the cuprates ${ }^{108}$. Numerics have revealed key physical insights, but face challenges extending to large system sizes, very low temperatures, or beyond local observables ${ }^{109}$. Mean-field approaches may be able to capture general qualitative features of the phase diagram, but are not designed to identify microscopic features of the wavefunction ${ }^{\text {IIo, III }}$. While these three approaches have provided and continue to provide key insights, quantum gas microscopy is naturally suited to assess a fourth category of techniques: microscopic theoretical approaches. These theories take a "bottom-up" approach in that they target a treatment of the quantum mechanical wavefunction such that the resulting macroscopic physics is as expected.

For example, it is understood that in the doped Hubbard model, dopant delocalization for kinetic energy minimization competes with spin interactions in the background antiferromagnet. The result is a general loss of antiferromagnetic order, however the exact microscopic mechanism is still not agreed upon. We examine two microscopic theories which may describe the doped Hubbard 
model in our experimental temperature regime of temperatures $T$ between $0.5 \mathrm{~J}$ and $0.7 \mathrm{~J}$ : geometric strings and $\pi$-flux states.

\section{I.I $\pi$-FLUX THEORY}

The $\pi$-flux theory stems from Anderson's resonating valence bond (RVB) picture ${ }^{\mathrm{II} 2}$, which considers trial wavefunctions of free holes moving through a spin liquid comprised of singlet coverings. These trial wavefunctions are a good approach because it is known that any $S U(2)$ invariant state can be written as a superposition of singlet coverings ${ }^{31}$. Here we consider one particular class of RVB wavefunctions which have been studied extensively for their low variational energy, called $\pi$ flux states ${ }^{\mathrm{IIO}}$. We obtain these wavefunctions by beginning with the ground state $\left|\Psi_{\mathrm{MF}}(\pi)\right\rangle$ of the mean-field Hamiltonian:

$$
\begin{aligned}
\hat{\mathcal{H}}_{\mathrm{MF}}= & -\frac{1}{2} J^{*} \sum_{\mathrm{i} \in A} \sum_{\sigma}\left(e^{i \Phi / 4} \hat{c}_{\mathrm{i}, \sigma}^{\dagger} \hat{c}_{\mathrm{i}+\mathrm{x}, \sigma}+e^{-i \Phi / 4} \hat{c}_{\mathrm{i}, \sigma}^{\dagger} \hat{c}_{\mathrm{i}+\mathrm{y}, \sigma}+\text { h.c. }\right) \\
& -\frac{1}{2} J^{*} \sum_{\mathrm{i} \in B} \sum_{\sigma}\left(e^{-i \Phi / 4} \hat{c}_{\mathrm{i}, \sigma}^{\dagger} \hat{c}_{\mathrm{i}+\mathrm{x}, \sigma}+e^{i \Phi / 4} \hat{c}_{\mathrm{i}, \sigma}^{\dagger} \hat{c}_{\mathrm{i}+\mathrm{y}, \sigma}+\text { h.c. }\right)
\end{aligned}
$$

Here, $\mathrm{i} \in A(B)$ denotes lattice sites $\mathrm{i}$ which are part of the $\mathrm{A}(\mathrm{B})$ sublattice, $\hat{c}_{\mathrm{i}, \sigma}^{(\dagger)}$ is the annihilation (creation) operator of a fermion with spin $\sigma$, and $\mathrm{x}(\mathrm{y})$ is the lattice unit vector in the $x(y)$ direction. This Hamiltonian describes fermions with nearest neighbor tunneling amplitude $J^{*} / 2$ and staggered flux $\pm \Phi$ per plaquette, as seen in figure 4.Ia. We consider the case $\Phi=\pi$, from which the $\pi$-flux state gets its name ${ }^{\mathrm{II} 3}$. This case is special not only because it preserves the lattice translational symmetry, but also because its eigenvalues are identical to that of the $d$-wave superconductor ${ }^{\mathrm{II} 4}$. 


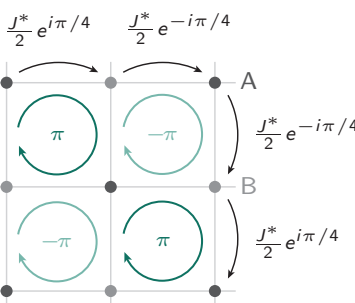

C

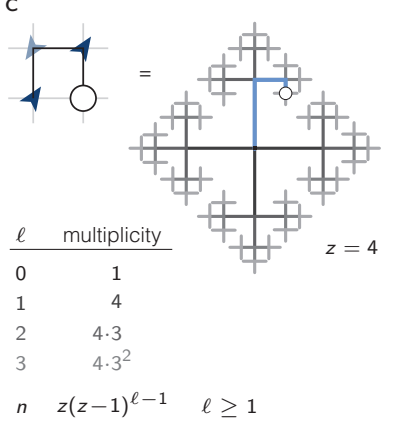

b

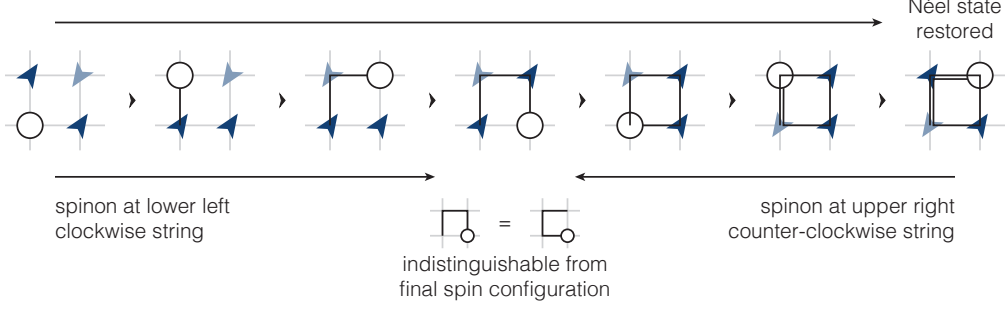

d

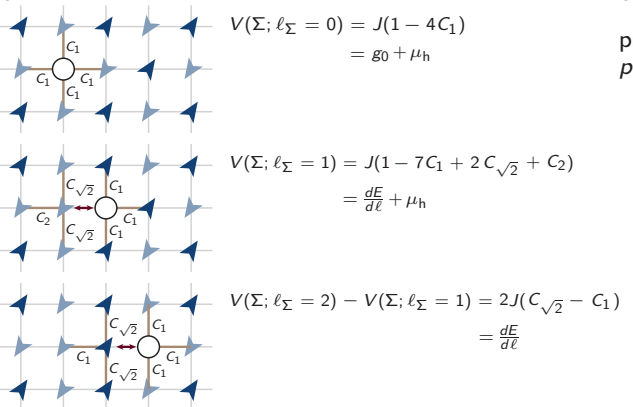

e

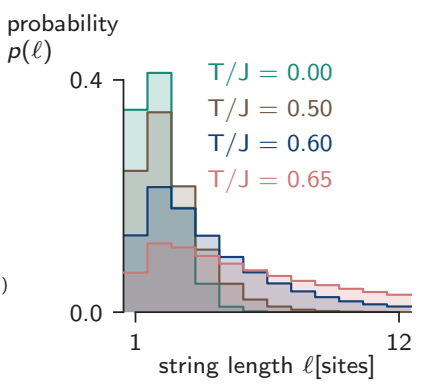

Figure 4.1: Microscopic theories of the doped Hubbard model. a, Staggered flux lattice for $\pi$-flux states. The lattice with $\pm \pi$ flux per plaquette yields states with a low variational energy, making them of interest. $\mathbf{b}$, Trugman loop example in the context of geometric string theory. Here a hole can restore the original Néel state by traversing a square one and a half times. Equivalently, two holes at differing starting positions and the same Néel state can create the same end configuration, yielding two indistinguishable string states. These Trugman loops are omitted to create an approximately orthonormal basis of string states. c, Mapping of string state from square to Bethe lattice. The mutiplicity of states of length $\ell$ increases exponentially in $\ell$. d, Derivation of string potential. By propagating a chargon and considering its new and former nearest-neighbor interactions, its potential can be determined. e, Geometric string length histogram for several temperatures, derived from geometric string theory.

Fourier transforming leads to

$$
\hat{\mathcal{H}}_{\mathrm{MF}}=\sum_{\mathrm{k} \in \mathrm{MBZ}, \sigma}\left(\begin{array}{ll}
\hat{c}_{\mathrm{k}, \sigma}^{\dagger} & \hat{c}_{\mathrm{k}+\mathrm{G}, \sigma}^{\dagger}
\end{array}\right) \hat{h}_{\mathrm{k}}\left(\begin{array}{c}
\hat{c}_{\mathrm{k}, \sigma} \\
\hat{c}_{\mathrm{k}+\mathrm{G}, \sigma}
\end{array}\right)
$$


with

$$
\begin{aligned}
\hat{h}_{\mathrm{k}} & =\operatorname{Re} R(\mathrm{k}) \hat{\tau}^{z}+\operatorname{Im} R(\mathrm{k}) \hat{\tau}^{y} \\
R(\mathrm{k}) & =-J^{*}\left(\cos k_{x} e^{i \theta_{0}}+\cos k_{y} e^{-i \theta_{0}}\right),
\end{aligned}
$$

and Pauli matrices $\hat{\tau}=\left(\hat{\tau}^{x}, \hat{\tau}^{y}, \hat{\tau}^{z}\right)$. Here, $\mathrm{k} \in \mathrm{MBZ}$ denotes momenta $\mathrm{k}=\left(k_{x}, k_{y}\right)$ in the magnetic Brillouin zone. Diagonalizing $\hat{h}_{\mathrm{k}}$ leads to two eigenstates $\left|u_{\mathrm{k}, \mu}\right\rangle=\left(u_{\mathrm{k}, \mu}^{0}, u_{\mathrm{k}, \mu}^{1}\right)^{T}$ for every momentum $\mathrm{k}$ in the magnetic Brillouin zone. Bloch's theorem yields the $\pi$-flux state wavefunction

$$
\psi_{\mathrm{k}, \mu}(\mathrm{r})=\frac{1}{\sqrt{L^{2}}}\left(u_{\mathrm{k}, \mu}^{0}+u_{\mathrm{k}, \mu}^{1} e^{i \mathrm{Gr}}\right) e^{i \mathrm{kr}}
$$

with band index $\mu= \pm$ and a system size of $L$ by $L$ sites.

Now we consider the Gutzwiller projected thermal density matrix $\hat{\rho}=\mathcal{P}_{\mathrm{GW}} e^{-\hat{\mathcal{H}}_{\mathrm{MF}} / k_{\mathrm{B}} T} \mathcal{P}_{\mathrm{GW}}$ for this Hamiltonian. From this density matrix, we use Metropolis Monte Carlo sampling to obtain Fock states of fermions. More specifically, we start from a random occupation of states in momentum space for both fermionic spin species as well as a random configuration without double occupancies in real space. Our system size is 16 by 16 sites and for a given doping value, we assume a spin balanced system. From any given state, updates in real space as well as updates in momentum space for up-spin and down-spin fermions are possible. In real space, two neighboring sites can exchange their occupation if they differ. In momentum space, a given fermion can change its momentum to any other unoccupied momentum. Note that in momentum space, we treat up and down fermions 
separately from each other such that two fermions of opposite spin can have the same momentum. Updates to the snapshots are then accepted or rejected according to the probability distribution

$$
p_{\beta}\left(\alpha_{\mathrm{r}}, \alpha_{\mathrm{k}}\right)=e^{-\beta E\left(\alpha_{\mathrm{k}}\right)}\left|\left\langle\alpha_{\mathrm{r}} \mid \alpha_{\mathrm{k}}\right\rangle\right|^{2},
$$

where $\beta=\left(k_{B} T\right)^{-1}$ is the inverse temperature and $\left|\alpha_{\mathrm{r}(\mathrm{k})}\right\rangle$ denote Fock states in configuration (momentum) space. Note that while equation (4.6) is not normalized, the normalization does not matter for the Metropolis sampling, since only ratios of probability distributions are required. The energy of the state $\left|\alpha_{\mathrm{k}}\right\rangle$ is given by

$$
E\left(\alpha_{\mathrm{k}}\right)=\sum_{\mathrm{k} \text { occ. in } \alpha_{\mathrm{k}}} \epsilon(\mathrm{k})
$$

with eigenenergies $\epsilon(\mathrm{k})$ of $\hat{h}_{\mathrm{k}}$ and the sum is taken over momenta $\mathrm{k}$ which are occupied in the considered Fock state $\left|\alpha_{\mathrm{k}}\right\rangle$.

After generating a sample of several thousand Fock states $\alpha_{r}$, doublon-hole pairs are artificially added with a probability given by $4 t^{2} / U^{2}$ on nearest-neighbor sites with opposite spins. Note that the probability can also be determined through determinantal quantum Monte Carlo (dQMC) simulation; for $U / t=8$ this yields a slightly higher percentage of states occupied by doublon-hole pairs of about $8 \%$. After the snapshots have been simulated, we cut out a circular region of interest of the same size as in the experiment to obtain the same boundary effects in both cases. Furthermore, we simulate the experimental imaging procedure and keep the parity-projected density distribution 
of either both spins or with one spin state removed. The coupling $J^{*}=3 J$ in the mean-field Hamiltonian is chosen such that at half filling, the nearest- and diagonal next-nearest-neighbor spin correlators obtained from the simulation at the temperature $T=0.6 \mathrm{~J}$ fit our experimental data as closely as possible. Without any other fitting parameter, the doping dependence of the nearest neighbor spin correlator is described correctly. However, the temperature dependence of the spin correlators even at half filling is not captured correctly with these parameters.

\section{I.2 GeOMETRIC STRING THEORY}

Geometric string theory begins by considering the motion of a single hole in an antiferromagnetic background. However, the question of what happens to a hole placed into a quantum antiferromagnet is not a new one. In 1968, Bulaevskii, Nagaev, and Khomskii discussed the motion of an extra electron (equivalent to an extra hole, due to particle-hole symmetry in the Hubbard model) in an antiferromagnetic at half-filling. They noted how as the electron moves away from an initial position, flipped spins appear on its trajectory and the total exchange energy increases with the length of the trajectory ${ }^{\mathrm{II}}$. Because of this increasing energy, the electron is "auto-localized", much like a harmonic oscillator. They also draw a clear distinction to the polaron in that the magnetic-polaron state exhibits a non-zero magnetic moment of the region where the electron is localized, regardless of where it is localized; by contrast, here there is no additional magnetic moment, and the magnetic ordering may or may not be disturbed depending on the electron position. Two years later, Brinkman and Rice extended these ideas by quantifying the effect of the possible particle trajectories and resulting spin configurations, calculating the density of states and an approximate single-particle Green's 
function ${ }^{\mathrm{II}}$. By 1996, the idea of a composite charge (holon) and spin (spinon) quasiparticle was introduced in the $t-J$ model ${ }^{\mathrm{II}}$. In particular, Beran, Poilblanc, and Laughlin performed exact calculations for small system sizes and found evidence for fractionalization of the injected hole into spinon and holon quasiparticles, with the two bound together by an attractive interaction which obeys a "string law". They also noted the differences between $\mathrm{ID}$ and $2 \mathrm{D}$ with respect to how these two elementary excitations may interact with one another.

The most recent work on the bound quasiparticles is by Grusdt, Bohrdt, and Demler, and uses geometric strings to describe magnetic polarons ${ }^{\mathrm{II} 8}$. Here the fast motion of the chargon (referred to as the "holon" in previous works) creates a fluctuating lattice geometry which can be detected in projective measurement by a local disturbance in the antiferromagnetic spin ordering. They begin with the $2 \mathrm{D} t-J$ model:

$$
\hat{\mathcal{H}}_{t-J}=-t \sum_{\sigma=\uparrow, \downarrow} \sum_{\langle i, j\rangle} \hat{\mathcal{P}}_{\mathrm{GW}}\left(\hat{c}_{\mathrm{i}, \sigma}^{\dagger} \hat{c}_{\mathrm{j}, \sigma}+\text { h.c. }\right) \hat{\mathcal{P}}_{\mathrm{GW}}+J \sum_{\langle\mathrm{i}, \mathrm{j}\rangle}\left(\hat{\mathrm{S}}_{\mathrm{i}} \cdot \hat{\mathrm{S}}_{\mathrm{j}}-\frac{1}{4} \hat{n}_{\mathrm{i}} \hat{n}_{\mathrm{j}}\right)
$$

where the Gutzwiller projection is explicitly included to restrict the Hilbert space to no more than one particle per site. As a result, at half-filling there is exactly one particle per site, the tunneling term plays no role, and the ground state wavefunction is that of the interaction term of the Hamiltonian, $\left|\Psi_{0}\right\rangle$. We can then define the bosonic chargon and $S=1 / 2$ fermionic spinon operators $\hat{h}_{\mathrm{j}}$ and $\hat{f}_{\mathrm{j}, \sigma}$, respectively, where $\hat{c}_{\mathrm{j}, \sigma}=\hat{h}_{\mathrm{j}}^{\dagger} \hat{f}_{\mathrm{j}, \sigma}$. Then by introducing a hole into the half-filled ground state, we get:

$$
\hat{h}_{\mathbf{j}^{s}}^{\dagger} \hat{f}_{\mathfrak{j}^{s}, \bar{\sigma}}\left|\Psi_{0}\right\rangle=:\left|\mathbf{j}^{s}, \sigma, 0\right\rangle
$$


where $\bar{\sigma}$ reverses the spin $\sigma$. This is equivalent to removing a charge from site $\mathrm{j}^{s}$ and reversing its spin, creating a spinon and chargon on that lattice site.

Because chargon fluctuations occur on a much faster timescale than spinon fluctuations, we apply a Born-Oppenheimer approximation and first fix the spinon to examine the chargon physics. In the full treatment, the spinon fluctuations are eventually considered ${ }^{\mathrm{II}}$; however, here we need only the distribution of geometric string lengths, so we will not need to consider spinon motion. We also make a frozen spin approximation, wherein the motion of the chargon displaces the spins along its path, but otherwise keeps their quantum state and entanglement with other spins unperturbed. In other words, one can label every spin in the quantum state; then, as the chargon moves, the labels of the spins change but the quantum state is otherwise unmodified.

Under these approximations, after the chargon moves along a string $\Sigma$ we get the state

$$
\left|j^{s}, \sigma, \Sigma\right\rangle=\hat{G}_{\Sigma} \hat{h}_{\mathbf{j}^{s}}^{\dagger} \hat{f}_{\mathfrak{j}^{s}, \bar{\sigma}}\left|\Psi_{0}\right\rangle
$$

The string operator $\hat{G}_{\Sigma}$ swaps the position of the chargon at $\mathrm{j}$ with a spin at $\mathrm{i}$ for every link $\langle\mathrm{i}, \mathrm{j}\rangle$ in the string $\Sigma$, starting with the chargon at the spinon position $j^{s}$ :

$$
\hat{G}_{\Sigma}=\prod_{\langle\mathrm{i}, j\rangle \in \Sigma}\left(\hat{h}_{\mathrm{i}}^{\dagger} \hat{h}_{\mathrm{j}} \sum_{\tau=\uparrow, \downarrow} \hat{f}_{\mathrm{j}, \tau}^{\dagger} \hat{f}_{\mathrm{i}, \tau}\right)
$$

Now we make a third approximation, which is that these states $\left|j^{s}, \sigma, \Sigma\right\rangle$ constitute an orthonormal basis. This requires disregarding so-called Trugman loops ${ }^{\text {I9 }}$, where a hole retraces its path in a cir- 
cular fashion and in doing so restores the original spin background, for example as shown in 4.rb. However, the effect of these loops seems to be $\operatorname{minor}^{\mathrm{I2O}}$, and the overlap between differing string states seems limited to several percent ${ }^{\mathrm{II}}$.

The string state basis consists of strings where the chargon does not move backwards. Because of this, these strings can be better described using a Bethe lattice, or Cayley graph, rather than a square lattice, as shown in figure 4.Ic. The effective Hamiltonian then consists of a tunneling term for chargon motion, as well as a potential energy term $\hat{\mathcal{H}}_{J}^{\Sigma}$. This term captures the "auto-localizing" as introduced by Bulaevskii, Nagaev, and Khomskiǐ; in other words, it captures the energy cost for distorting the spin configuration around the spinon site $j^{s}$ :

$$
\hat{\mathcal{H}}_{J}^{\Sigma}=\sum_{\mathbf{j}, \sigma, \Sigma}\left\langle\mathbf{j}^{s}, \sigma, \Sigma\left|\hat{\mathcal{H}}_{J}\right| \mathbf{j}^{s}, \sigma, \Sigma\right\rangle \times\left|\mathbf{j}^{s}, \sigma, \Sigma\right\rangle\left\langle\mathbf{j}^{s}, \sigma, \Sigma\right|
$$

The next step, then, is to calculate the energy $\left\langle j^{s}, \sigma, \Sigma\left|\hat{\mathcal{H}}_{J}\right| \mathbf{j}^{s}, \sigma, \Sigma\right\rangle=: V(\Sigma)$.

We calculate this energy by considering the distortions caused by moving a chargon. First, the introduction of a hole already shifts the energy by $J\left(1-4 C_{s}(1)\right)$, where $C_{s}(d)$ is the spin correlator at distance $d$, as depicted in figure 4.Id. The first term comes from the density-density term of the Hamiltonian 4.8, and the second comes from the four nearest-neighbor bonds that have been removed. Note that we assume a lattice with isotropic tunneling and therefore isotropic correlation strengths. As the chargon moves away from its initial site $j^{s}$, it displaces a spin and breaks another three nearest-neighbor bonds of total energy $3 J C_{s}(1)$. In addition, the displaced spin now sits next to two spins that were previously diagonal next-nearest neighbors and on spin that was previously a 
staight next-nearest neighbor. This introduces an additional energy cost of $2 J C_{s}(\sqrt{2})+J C_{s}(2)$, such that strings of length $\mathrm{I}$ have a total energy cost of $J\left(1-7 C_{s}(1)+2 C_{s}(\sqrt{2})+C_{s}(2)\right)$. As the chargon continues to move from one site to the next, it displaces spins and separates them from two nearest neighbors, placing them next to two diagonal next-nearest neighbors and yielding an additional energy $d E / d \ell=2 J\left(C_{s}(\sqrt{2})-C_{s}(1)\right)$ per unit length of the string. Therefore, we make a fourth approximation in that the energy depends linearly on the length $\ell_{\Sigma}$ of the string. The potential is then:

$$
V(\Sigma) \approx \frac{d E}{d \ell} \ell_{\Sigma}+g_{0} \delta_{\ell_{\Sigma}, 0}+\mu_{\mathrm{h}}
$$

where

$$
\begin{aligned}
\frac{d E}{d \ell} & =2 J\left(C_{\sqrt{2}}-C_{1}\right) \\
g_{0} & =-J\left(C_{2}-C_{1}\right) \\
\mu_{h} & =J\left(1+C_{2}-5 C_{1}\right)
\end{aligned}
$$

and we have abbreviated the spin correlator $C_{s}(d)$ by $C_{d}$, which will be done for the rest of this section. Note that if the string has a corner, then the energy cost for that corner becomes $\frac{\widetilde{d E}}{d \ell}=$ $J\left(C_{\sqrt{2}}+C_{2}-2 C_{1}\right)$, where for our coldest temperatures $C_{2} \approx 0.8 C_{\sqrt{2}}$ and $\frac{\widetilde{d E}}{d \ell} \approx 0.97 \frac{d E}{d \ell}$ which is a small effect. In addition to assuming a linear dependence of energy on string length, the turns of the string are neglected, i.e. we assume rotational symmetry on the Bethe lattice, equivalent to only considering the rotational ground state. Because of this, we can straightforwardly find an effective 
ID model for the Hamiltonian.

To find the ID Hamiltonian, we first compute the normalization between the string states $\left|j^{s}, \sigma, \Sigma\right\rangle$ of length $\ell_{\Sigma}$ and the $\mathrm{ID}$ wavefunction $\phi_{\ell}$. In other words, while there can be many strings on the Bethe lattice of length $\ell_{\Sigma}$, for the corresponding $\mathrm{ID}$ Hamiltonian there is only one, so it must be scaled accordingly. It is straightforward to see that for $\ell_{\Sigma}=0$, there is one string on the Bethe lattice; for $\ell_{\Sigma}=1$, there are four strings on the Bethe lattice; and for every $\ell_{\Sigma}>1$, increasing the length by one increases the number of strings on the Bethe lattice by a factor of three. Noting that this comes from the coordination number $z=4$ of the square lattice, we find:

$$
\left|j^{s}, \sigma, \Sigma\right\rangle=\sqrt{\frac{(z-1)^{\left(1-\ell_{\Sigma}\right)}}{z}} \phi_{\ell}, \quad \ell_{\Sigma} \geq 1
$$

such that $\sum_{\Sigma}\left\langle j^{s}, \sigma, \Sigma \mid j^{s}, \sigma, \Sigma\right\rangle=\sum_{\ell=0}^{\infty}\left|\phi_{\ell}\right|^{2}$. The resulting Schrödinger equation becomes:

$$
\begin{aligned}
& E \phi_{0}=-t^{*} \sqrt{\frac{z}{z-1}} \phi_{1}+V_{0} \phi_{0} \\
& E \phi_{1}=-t^{*}\left(\phi_{2}+\sqrt{\frac{z}{z-1}} \phi_{0}\right)+V_{1} \phi_{1} \\
& E \phi_{\ell}=-t^{*}\left(\phi_{\ell+1}+\phi_{\ell-1}\right)+V_{\ell_{\Sigma}} \phi_{\ell}, \quad \ell_{\Sigma} \geq 2
\end{aligned}
$$

where $t^{*}=t \sqrt{z-1}$ and $V_{\ell_{\Sigma}}=V\left(\Sigma ;|\Sigma|=\ell_{\Sigma}\right)$.

To obtain an analytic form for the string eigenfunctions and eigenenergies, we now consider the continuum limit where instead of discrete lengths $\ell_{\Sigma} \geq 0$ we use a continuous variable $x \geq 0$. We also omit the additional tunneling renormalizations required for short string lengths, seen in 
equations 4.I8 and 4.19. This gives us, starting from equation 4.20:

$$
\begin{aligned}
E \phi_{\ell}= & -t^{*}\left(\phi_{\ell+1}-2 \phi_{\ell}+\phi_{\ell-1}\right)+\left(V_{\ell_{\Sigma}}-2 t^{*}\right) \phi_{\ell} \\
E \phi(x)= & -t^{*} \partial_{x}^{2} \phi(x)+ \\
& \left(2 J\left(C_{\sqrt{2}}-C_{1}\right) x-J\left(C_{2}-C_{1}\right) \delta_{x, 0}+J\left(1+C_{2}-5 C_{1}\right)-2 t^{*}\right) \phi(x) \\
E \phi(x)= & \left(-\frac{\partial_{x}^{2}}{2 m^{*}}+V(x)\right) \phi(x)
\end{aligned}
$$

Here $m^{*}=1 / 2 t^{*}$ and $V(x)=\left(2 J\left(C_{\sqrt{2}}-C_{1}\right) x+J\left(1+C_{2}-5 C_{1}\right)-2 t^{*}\right)$. Notice that we omit the delta function potential at $x=0$ for simplicity. The result is a one-sided linear potential with a fixed energy offset of $-2 t^{*}$. This equation is an Airy equation, whose solutions are Airy functions and whose eigenvalues $E_{n}$ are given by:

$$
\begin{aligned}
E_{n} & =a_{n}\left(\frac{\left(2 J\left(C_{\sqrt{2}}-C_{1}\right)\right)^{2}}{2 m^{*}}\right)^{1 / 3}-2 t^{*} \\
& =a_{n}\left(2\left(C_{\sqrt{2}}-C_{1}\right)\right)^{2 / 3}(z-1)^{1 / 6} J^{2 / 3} t^{1 / 3}-2 t \sqrt{z-1}
\end{aligned}
$$

for numerical coefficients $a_{n}$.

Finally, we can use our lattice coordination number $z=4$, experimental Hubbard parameter value of $t=2 J$, and values of spin correlators $C_{\sqrt{2}}$ and $C_{1}$ for a given temperature to determine the energy spectrum at that temperature. Each Airy function gives rise to a distribution of string length probability amplitudes, which can then be weighted by the Boltzmann factor. However, this derivation has assumed the rotational ground state, whereas rotational excitations must also be 
included in the thermal distribution of states. This can be done numerically to obtain geometricstring length distributions at any temperature; I plot the distributions for several temperatures in figure 4.Ie.

A few remarks are in order. First, the use of the $t-J$ model is to focus on the regime where interactions are very large such that doublon-hole pairs can be neglected. Other than this effect, the remaining calculations do not specifically require the $t-J$ model over the Hubbard model, and thus we use the derived string length distribution in our simulations for the geometric string theory in the Hubbard model. Second, while this treatment considers a single hole in an antiferromagnetic background, for sufficiently few holes there should not be interactions between the holes. However, this assumption will break down at large doping or at sufficiently low temperatures where correlations may arise. Third, while spinon dynamics are expected, albeit at a slower timescale relative to chargon motion, we do not need to consider these dynamics because they will result in the center-ofmass motion of the string, to which we are not sensitive. Fourth, to briefly contextualize this result, the $J^{2 / 3} t^{1 / 3}$ scaling of the energy has also been seen in various numerical studies for a wide range

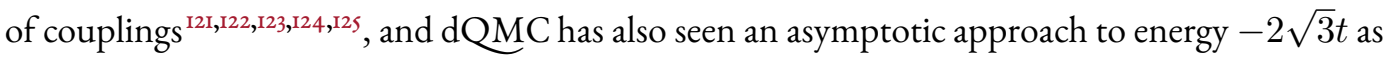
$J \rightarrow 0^{\mathrm{I2} 5}$. Beyond this, I defer to other work ${ }^{\mathrm{I20}, \mathrm{II} 8}$ for a full treatment and contextualization of geometric strings and a microscopic spinon-chargon theory of magnetic polarons.

Finally, geometric strings are a way to introduce hidden order. Hidden order arises in many-body systems when individual configurations are each characterized by a particular pattern, but the average over these configurations leads to an apparent loss of order. In contrast, instantaneous projective measurements have the potential to reveal these underlying patterns. This can be better demon- 
strated by considering the ID Hubbard model at strong coupling ${ }^{\mathrm{I} 26, \mathrm{I} 27}$. Here, individual configurations are characterized by dopants within magnetically ordered chains, but the average over these configurations show two-point spin correlations which decay more rapidly with distance. This apparent loss of magnetic order is in fact hidden order, hidden by the dopants and their varying positions $^{\mathrm{I28,129}}$. Although direct detection of this hidden string order remains inaccessible in solids, experiments with ultracold atoms enable projective measurements, or "snapshots", and generally can provide access to such structures ${ }^{\mathrm{I} 3}$. While the hidden order in $\mathrm{ID}$ is well understood, the physics of the $2 \mathrm{D}$ Hubbard model is fundamentally more complex due to an intricate interplay between spin and charge degrees of freedom; as a result, formulating an appropriate correlation function to search for hidden order becomes significantly more challenging. Quantum gas microscopy, however, provides a new perspective beyond the framework of two- or multi-point correlations. Hidden string order can be searched for directly within individual snapshots of the quantum mechanical wavefunction, where quantum fluctuations are resolved, and is the focus of the remainder of the chapter.

\subsection{EXPERIMENTAL DATASET}

All experimental measurements were taken with $U / t=8.1(2)$ and a system size of approximately 80 sites. We vary both the temperature and the doping across a wide parameter range. The temperature spans between $0.50(4) J$ and $1.8(1) J$ with hotter temperatures achieved by holding the sample in the combined lattice and DMD potential. The system is also hole-doped up to a doping $\delta$ of 0.32 


\begin{tabular}{|c|c|c|c|c|c|c|c|c|c|c|c|c|c|c|c|c|c|}
\hline & \multicolumn{17}{|c|}{$\operatorname{doping}[\%]$} \\
\hline & 0 & 2 & 4 & 6 & 8 & 10 & 12 & 14 & 16 & 18 & 20 & 22 & 24 & 26 & 28 & 30 & 32 \\
\hline 0.5 & $\begin{array}{l}69 \\
{[34]}\end{array}$ & & & $\begin{array}{l}66 \\
{[32]}\end{array}$ & & & $\begin{array}{l}67 \\
{[33]}\end{array}$ & & & & & & $\begin{array}{l}67 \\
{[33]}\end{array}$ & & & & \\
\hline 0.6 & $\begin{array}{l}2224 \\
{[1122]}\end{array}$ & $\begin{array}{l}94 \\
{[47]}\end{array}$ & $\begin{array}{l}527 \\
{[264]}\end{array}$ & $\begin{array}{l}114 \\
{[57]}\end{array}$ & $\begin{array}{l}1072 \\
{[550]}\end{array}$ & $\begin{array}{l}511 \\
{[255]}\end{array}$ & $\begin{array}{l}277 \\
{[140]}\end{array}$ & $\begin{array}{l}262 \\
{[131]}\end{array}$ & & $\begin{array}{l}505 \\
{[253]}\end{array}$ & $\begin{array}{l}210 \\
{[103]}\end{array}$ & & $\begin{array}{l}148 \\
{[74]}\end{array}$ & $\begin{array}{l}209 \\
{[104]}\end{array}$ & $\begin{array}{l}358 \\
{[179]}\end{array}$ & $\begin{array}{l}149 \\
{[74]}\end{array}$ & $\begin{array}{l}208 \\
{[104]}\end{array}$ \\
\hline 0.7 & $\begin{array}{l}4571 \\
{[2380]}\end{array}$ & $\begin{array}{l}505 \\
{[256]}\end{array}$ & $\begin{array}{l}712 \\
{[357]}\end{array}$ & $\begin{array}{l}1610 \\
{[858]}\end{array}$ & $\begin{array}{l}1918 \\
{[1034]}\end{array}$ & $\begin{array}{l}1125 \\
{[564]}\end{array}$ & $\begin{array}{l}1023 \\
{[515]}\end{array}$ & $\begin{array}{l}450 \\
{[227]}\end{array}$ & $\begin{array}{l}401 \\
{[201]}\end{array}$ & $\begin{array}{l}886 \\
{[446]}\end{array}$ & $\begin{array}{l}375 \\
{[190]}\end{array}$ & & $\begin{array}{l}357 \\
{[181]}\end{array}$ & & $\begin{array}{l}353 \\
{[178]}\end{array}$ & $\begin{array}{l}372 \\
{[190]}\end{array}$ & \\
\hline 0.8 & $\begin{array}{l}296 \\
{[150]}\end{array}$ & & & $\begin{array}{l}78 \\
{[39]}\end{array}$ & & $\begin{array}{l}473 \\
{[238]}\end{array}$ & $\begin{array}{l}337 \\
{[169]}\end{array}$ & & & & & & & & & & \\
\hline 0.9 & $\begin{array}{l}346 \\
{[174]}\end{array}$ & & & & & $\begin{array}{l}191 \\
{[96]}\end{array}$ & $\begin{array}{l}854 \\
{[425]}\end{array}$ & & & & & & & & & & \\
\hline 1.0 & $\begin{array}{l}317 \\
{[160]}\end{array}$ & & & $\begin{array}{l}246 \\
{[123]}\end{array}$ & $\begin{array}{l}273 \\
{[137]}\end{array}$ & $\begin{array}{l}191 \\
{[94]}\end{array}$ & $\begin{array}{l}244 \\
{[122]}\end{array}$ & & & & & & & & & & \\
\hline 1.1 & $\begin{array}{l}358 \\
{[178]}\end{array}$ & & & $\begin{array}{l}76 \\
{[39]}\end{array}$ & $\begin{array}{l}370 \\
{[188]}\end{array}$ & $\begin{array}{l}485 \\
{[243]}\end{array}$ & $\begin{array}{l}134 \\
{[69]}\end{array}$ & & & & & & & & & & \\
\hline 1.2 & $\begin{array}{l}430 \\
{[217]}\end{array}$ & $\begin{array}{l}243 \\
{[123]}\end{array}$ & $\begin{array}{l}240 \\
{[120]}\end{array}$ & $\begin{array}{l}508 \\
{[258]}\end{array}$ & $\begin{array}{l}463 \\
{[234]}\end{array}$ & $\begin{array}{l}809 \\
{[403]}\end{array}$ & & & & & & & & & & & \\
\hline 1.3 & $\begin{array}{l}242 \\
{[123]}\end{array}$ & $\begin{array}{l}394 \\
{[200]}\end{array}$ & $\begin{array}{l}71 \\
{[35]}\end{array}$ & $\begin{array}{l}348 \\
{[177]}\end{array}$ & $\begin{array}{l}798 \\
{[399]}\end{array}$ & & & & & & & & & & & & \\
\hline 1.4 & & & & & & & & & & & & & & & & & \\
\hline 1.5 & & & $\begin{array}{l}209 \\
{[106]}\end{array}$ & $\begin{array}{l}147 \\
{[75]}\end{array}$ & $\begin{array}{l}102 \\
{[51]}\end{array}$ & $\begin{array}{l}108 \\
{[54]}\end{array}$ & & & $\begin{array}{l}150 \\
{[73]}\end{array}$ & & & & & & & & \\
\hline 1.6 & $\begin{array}{l}839 \\
{[453]}\end{array}$ & $\begin{array}{l}1276 \\
{[704]}\end{array}$ & $\begin{array}{l}930 \\
{[466]}\end{array}$ & & $\begin{array}{l}460 \\
{[231]}\end{array}$ & $\begin{array}{l}789 \\
{[469]}\end{array}$ & $\begin{array}{l}263 \\
{[131]}\end{array}$ & $\begin{array}{l}235 \\
{[116]}\end{array}$ & & & & & & & & & \\
\hline 1.7 & & $\begin{array}{l}289 \\
{[146]}\end{array}$ & $\begin{array}{l}1091 \\
{[648]}\end{array}$ & & & $\begin{array}{l}163 \\
{[103]}\end{array}$ & $\begin{array}{l}100 \\
{[52]}\end{array}$ & $\begin{array}{l}290 \\
{[143]}\end{array}$ & & & & & & & & & \\
\hline 1.8 & $\begin{array}{l}198 \\
{[100]}\end{array}$ & $\begin{array}{l}223 \\
{[112]}\end{array}$ & $\begin{array}{l}784 \\
{[397]}\end{array}$ & $\begin{array}{l}108 \\
{[51]}\end{array}$ & & & $\begin{array}{l}374 \\
{[187]}\end{array}$ & $\begin{array}{l}187 \\
{[92]}\end{array}$ & $\begin{array}{l}91 \\
{[46]}\end{array}$ & & & & & & & & \\
\hline
\end{tabular}

Table 4.1: Number of experimental realizations. For each doping value (columns) and temperature (rows, in units of $J$ ), this table lists the number of experimental realizations with spin removal and without (in brackets).

through varying the local chemical potential of the sample. We ensure that lattice loading timescales are set to those previously found to yield samples consistent with being in thermal equilibrium, and during imaging we selectively image one of the spin states or the total atom distribution ${ }^{19}$. The number of experimental realizations for each doping and temperature value is listed in table 4.I, grouped in doping bins of width 0.02 and temperature bins of $0.1 \mathrm{~J}$. Unless otherwise noted, all stated uncertainties and error bars denote the standard error of the mean. 


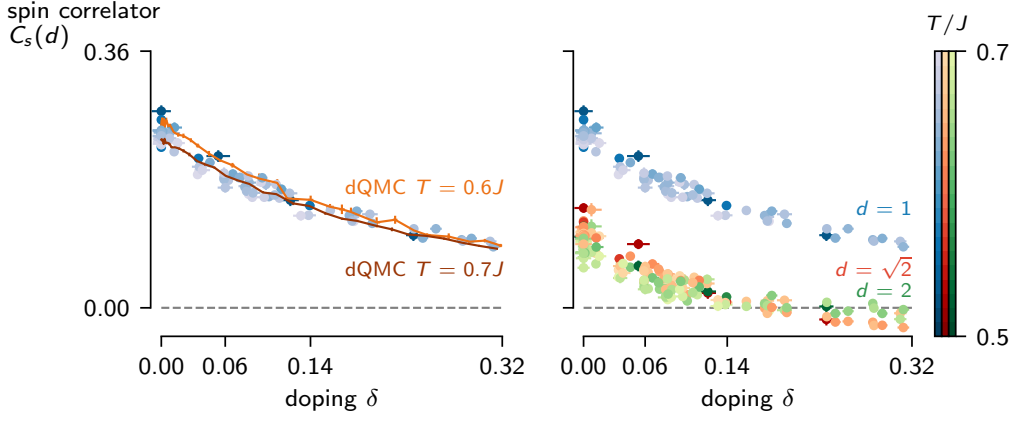

Figure 4.2: Temperature determination with the spin-spin correlator. a, Sign-corrected nearest-neighbor spin correlator versus doping from experiment and dQMC. Doping the system does not change the temperature beyond experimental uncertainty. b, First three correlation strengths for datasets prior to grouping by doping. The temperature is indicated by the colorbars.

\subsection{TEMPERATURE DETERMINATION}

When taking data, we interleave experimental realizations at half-filling and non-zero doping for the same hold time in the lattice. The temperature of the half-filling dataset can be determined by comparing the strength of the nearest-neighbor spin correlator with $\mathrm{dQM} \mathrm{C}^{\mathrm{I} 3 \mathrm{I}}$. We then assume that

changing the local chemical potential does not change the temperature significantly, which allows us to use the temperature of the half-filling sample for the doped datasets as well. This is a reasonable assumption, as seen in figure $4.2 \mathrm{a}$ through comparing the strength of the nearest-neighbor spin correlator for doped datasets with dQMC calculations. These calculations of the Hubbard model are on an $8 \times 8$ homogeneous square lattice using the Quantum Electron Simulation Toolbox ${ }^{\mathrm{I} 32,80}$. Indeed, agreement between the two indicate that our experimental approach to doping the system does not change the temperature of the sample beyond experimental uncertainty.

We combine all experimental realizations for temperatures between $0.50(4) \mathrm{J}$ and $0.70(3) \mathrm{J}$ for our "cold-temperature" dataset. The spread of temperatures is shown in the first three spin corre- 
lators $\tilde{C}_{s}(d)$ at distances $d$ of $1, \sqrt{2}$ and 2 in figure 4.2. The average temperature of these datasets, weighted by dataset size, is $T=0.65(4) \mathrm{J}$. Likewise, we combine all experimental realizations for temperatures between 1.3(1) $J$ and 1.8(1) $J$ for our "hot-temperature" dataset.

\subsubsection{DOpING DETERMINATION}

To determine the doping value from our measured singles density $n_{s}$, we use numerical simulations. For data between $T=0.6 J$ and $T=0.8 J$ we use data obtained from dQMC ${ }^{132,80}$, and for all larger temperatures we use data obtained from $\mathrm{NLCE}^{88}$. For $T<0.6 J$, the sign problem becomes significant. As a result, in this regime we use data at $T=0.6 \mathrm{~J}$, as the density sector of the equation of state is relatively insensitive to temperature here. We account for an imaging fidelity of $98.5 \%$. When statistical fluctuations cause the singles density to exceed the numerically-obtained singles density at half-filling, we treat those samples as at half-filling.

When determining the standard error of doping values for each experimental dataset, we assume that the particle density is linearly dependent on singles density. We apply a linear fit to doping versus singles density from the numerical simulation mentioned above, yielding approximately $\delta=1.22 \times\left(0.905-n_{s}\right)$, where $\delta$ is doping and $n_{s}$ is the singles density. We then calculate the standard error of the singles density and use the linear fit result to get the standard error of the mean doping value.

Since the actual doping value varies across datasets, we group datasets by their mean doping values within windows of width $2 \%$. This yields a single mean doping value $\bar{d}$ for the entire group. The associated uncertainty $\Delta$ is determined by assuming each dataset $k$ within the group was taken 
at a different doping value $d_{k}$ with a corresponding uncertainty $\delta d_{k}$. Then $\Delta$ can be calculated as:

$$
\Delta=\sqrt{\frac{1}{\sum_{k} n_{k}} \sum_{k}\left(\left(d_{k}-\bar{d}\right)^{2}+\delta d_{k}^{2}\right) n_{k}}
$$

For datasets which are sufficiently close to half-filling, fluctuations of additional holes or particles will both result in a decrease of the singles density. This single-sided cut-off of statistical fluctuations will lead to a systematic offset in the mean. To estimate this offset, we assume that the statistical fluctuations in total density follow a normal distribution centered at half filling with standard deviation $\sigma$. Then the resulting distribution in the singles density follows a half-normal distribution, characterized by an offset in the mean of $\sigma \sqrt{2 / \pi}$ and standard deviation of $\sigma \sqrt{1-2 / \pi}$. Note that this provides an upper bound of the systematic offset, because in reality our datasets are not all centered exactly at half filling. If we consider all datasets which are within one standard deviation of half filling, this results in an estimated systematic offset in the mean doping of the grouped dataset of about $0.25 \%$. This systematic uncertainty is included in the errorbar for half-filling doping values. We note that a higher-order correction to the dependence of the singles density on the total density makes the singles density less sensitive and therefore would only decrease the magnitude of this systematic error. 


\subsection{Analysis methodology}

\subsection{STRING-PATTERN DETECTION}

Every experimental snapshot is a projective measurement in the parity-projected Fock basis of the quantum mechanical many-body wavefunction. This measurement precludes a direct search of geometric strings, and it is not clear how one would be able to directly probe them. As a result, we put quite some effort into exploring indirect signatures of geometric strings which may be present in our data.

If we consider a classical picture, the antiferromagnet is a perfect checkerboard and as a hole moves around, it displaces each spin on its path onto the opposite-spin sublattice. Therefore, classical geometric strings can be found by searching for the sites which deviate from the background checkerboard. Quantum mechanically, the situation becomes much more complicated. First, the quantum antiferromagnetic exhibits several types of fluctuations which will cause deviations from a classical checkerboard. These include the underlying $S U(2)$ symmetry of the Hamiltonian, doublonhole pairs which arise when the interaction energy is large but not infinite, and thermal fluctuations from non-zero temperature. The largest of these effects is the first, where the staggered magnetization after a projective measurement can fluctuate drastically. Second, the current experiment measures the charge parity on every site, rendering doubly occupied sites and unoccupied sites indistinguishable. For images where we remove one spin species prior to imaging, these sites also become indistinguishable from sites that were initially occupied with a removed spin. In short, our half-filling 
background fluctuates and we cannot detect holes.

Acknowledging the additional physics introduced by having a quantum system, we developed a string-pattern detection algorithm motivated by the classical picture. It consists of three main steps: (i) postselection, to reduce the fluctuations associated with the $S U(2)$ symmetry of the system; (ii) determination of sites which deviate from a checkerboard pattern; and (iii) extraction of string patterns from those sites which deviate, according to the rules described in the main text. I now elaborate on the implementation of these steps.

In (i), we calculate the staggered magnetization of a circular region ("window") of diameter 7 sites (as shown in figure 4.3) as the region is scanned over the entire Io-site-diameter sample. For each image, we use the 7-site-diameter window of highest staggered magnetization. If there are multiple such possible regions, we take the upper-left-most one, however this is an arbitrary choice given that the entire sample is homogenous. Once all images have been reduced in size, we postselect on all data for the top $60 \%$ of the staggered magnetization.

In (ii), of the two possible checkerboard patterns, we select the one closer in staggered magnetization as reference for each image separately.

In (iii), we first sort the sites which deviate into disjoint sets, each of which consists of sites which can be connected by nearest neighbors. For each set, we identify all empty sites, as these may be the end of a geometric string. For each of these empty sites, we trace out all possible strings (sites which are connected via nearest neighbors to at most two other sites) and select the longest one. If there are multiple longest strings, we select one with the upper-left-most starting site. Again this is an arbitrary choice given the homogeneity of the system; we find that modifying this bias does not affect 


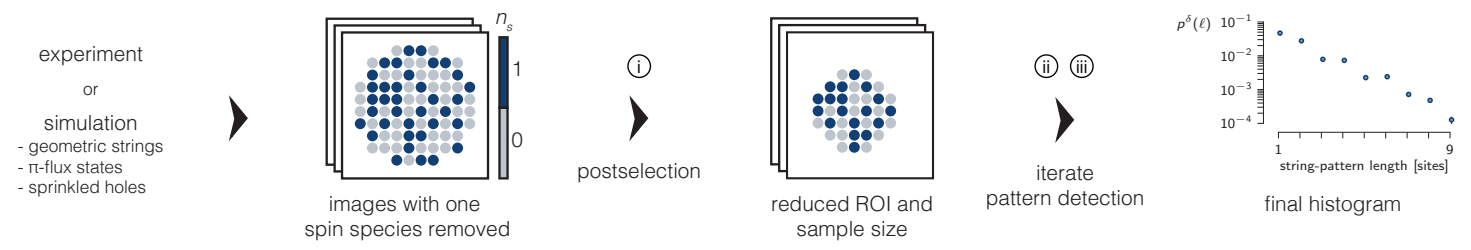

(i) postselection within each image and across dataset

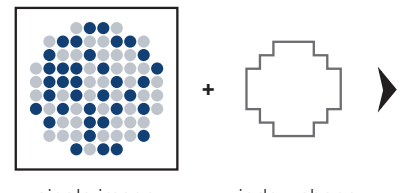

single image

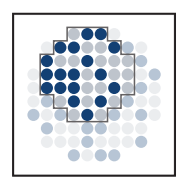

scan window across imag

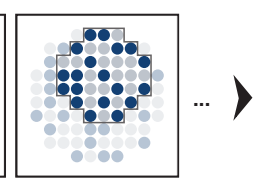

select window with highest staggered magnetization

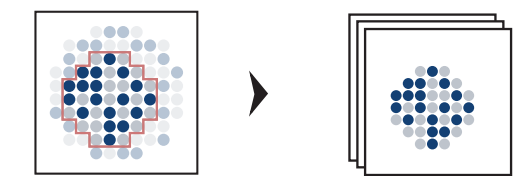

postselect on all data for top $60 \%$ staggered magnetization (ii) comparison to reference checkerboard

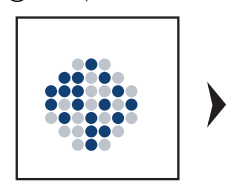

single image

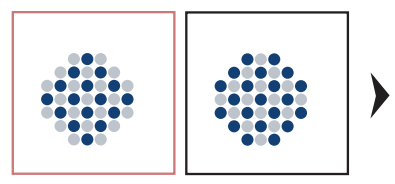

find closer reference pattern

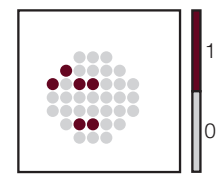

difference from reference checkerboard (ii) identification of string patterns from difference matrix

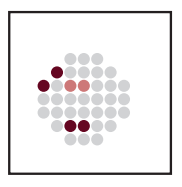

identify longest string pattern

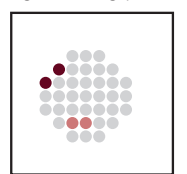

identify longest string pattern

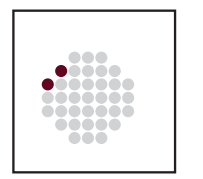

no more string patterns

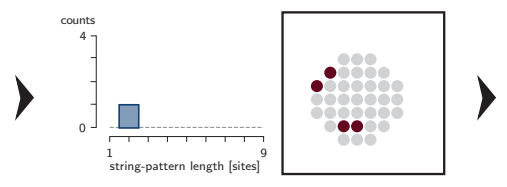

count string pattern \& remove

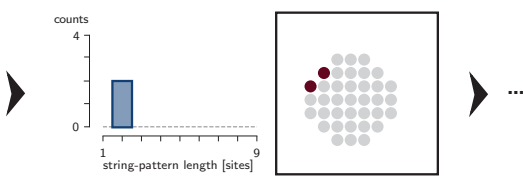

count string pattern \& remove

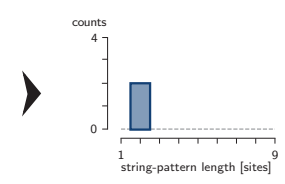

single image histogram

Figure 4.3: String detection algorithm. The string detection algorithm only uses images with one spin removed, but these images can come from experiment or one of the simulations. After postselection, the deviation from a reference checkerboard pattern is used to identify string patterns. String patterns consist only of sites which deviate from the reference checkerboard and must have an unoccupied site at one end, consistent with having a hole at that site. Therefore, after all possible string patterns have been counted and removed, there may be remaining sites which deviate from the reference checkerboard but do not contribute to the string pattern observables. 
the resulting string-pattern length distribution. The sites which are part of the identified string pattern are then removed from the set and the process is repeated until no more patterns can be found, i.e. the set no longer contains empty sites. This process is then repeated for each of the disjoint sets. In computing the lengths of these extracted string patterns, we use the number of sites in the pattern, which includes the site occupied by the hole, whereas in the geometric string formalism of section 4.I.2 the site occupied by the hole is not counted in the geometric string length.

We note that overlaps of strings or loops within strings are not treated correctly, because sites that do not deviate from the reference state are not taken into account. However, given the readout in the Fock basis, other algorithms will be similar in this regard. While these string patterns may be used to compare theories with experiment, we also carefully scrutinize the usefulness of the observables associated with these patterns as will be discussed throughout this chapter. In particular, the string patterns found through this algorithm do not necessarily directly correspond to geometric strings, as will be shown in section 4.4 .

\subsubsection{Simulation of microscopic theories}

The pattern finding algorithm yields string patterns which are not identical to geometric strings due to our use of a reference checkerboard to approximate the quantum antiferromagnetic background, as well as due to the possibility of string overlaps and loops. As a result, we cannot directly compare the measured string length distribution to the predictions from geometric-string theory. We therefore instead use these predictions to simulate snapshots, which in turn are analyzed to obtain string length distributions which can be directly compared. Because this theory makes no statement about 
the parent antiferromagnet, in this simulation the experimental images taken at half filling are used as the parent antiferromagnet. A number of holes corresponding to the desired doping value are then placed at random positions into these experimental images. For each hole, a length is sampled from the analytic string length distribution and the hole is propagated accordingly. The direction is chosen randomly at each step, but the hole cannot move backwards. This procedure produces a set of images which are then analyzed identically to the experimental dataset, such that the pattern detection scheme is common to both.

By introducing a snapshot-based simulation which can be analyzed in the same way as experimental data, we also enable comparisons to other theories that can produce snapshots. For example, we want to check that our string-pattern-based observables are sensitive to the effect of the motion of holes on an antiferromagnetic background, not just the addition of holes. Therefore, we create a set of snapshots with “sprinkled holes”, where a number of holes corresponding to a desired doping value is randomply placed into experimental half-filling images. Crucially, the holes are not propagated as they are in the geometric-string snapshots.

We also generate a set of snapshots for $\pi$-flux states. These snapshots do not rely on the experimental half-filling images, however the theory contains a free fit parameter which is determined through fitting the dependence of the experimental nearest-neighbor and diagonal next-nearestneighbor spin correlators on doping. After generating a set of I6-site by I6-site snapshots via Metropolis Monte Carlo sampling ${ }^{\mathrm{I} 33}$, we simulate the presence of doublon-hole pairs by replacing opposite spins on nearest-neighbor sites with a probability given by $4 t^{2} / U^{2}$. Numerics indicate that restricting doublon-hole pairs to nearest neighbors is a valid approximation in this regime ${ }^{87}$. Finally, the 


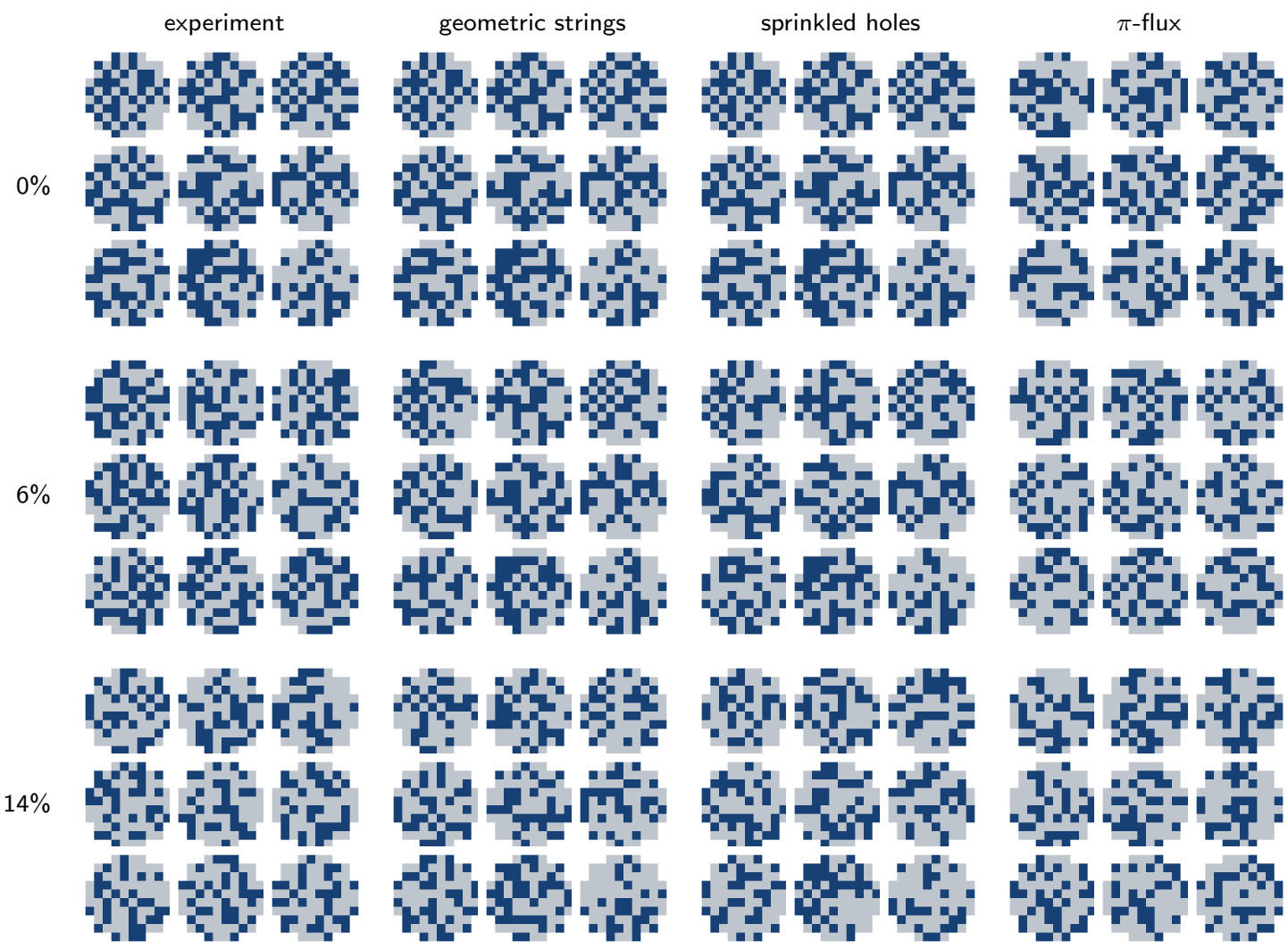

Figure 4.4: Example snapshots from experiment and simulation. Randomly selected single-spin-state images from experiment, geometric string theory, sprinkled holes, and $\pi$-flux states. Notice that at half-filling, images from experiment, geometric string theory, and sprinkled holes are identical by construction. Snapshots from doping values of $6 \%$ and $14 \%$ are also shown.

sample size is reduced to match the experimental sample size and the site occupations are converted to the parity-projected density distribution of either both spins or with one spin state removed. This mimics the boundary effects and imaging schemes of the experiment.

In figure 4.4 I show some randomly selected snapshots from the experiment, geometric strings, sprinkled holes, and $\pi$-flux states at several doping values, all with doublons and one of the two spin states removed, to provide an idea of what our datasets look like. 

probability $p^{\delta}(\ell)$

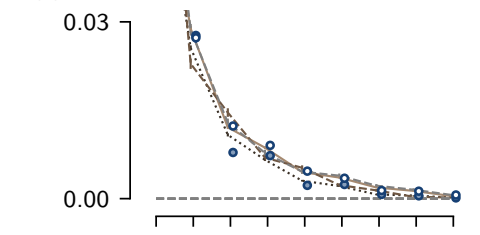

$p^{\delta}(\ell)$

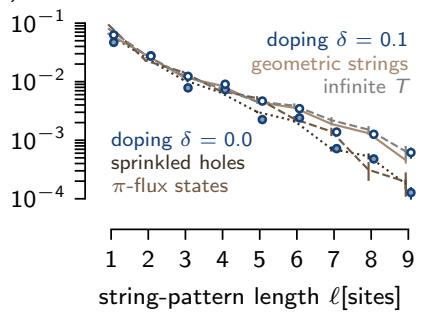

b
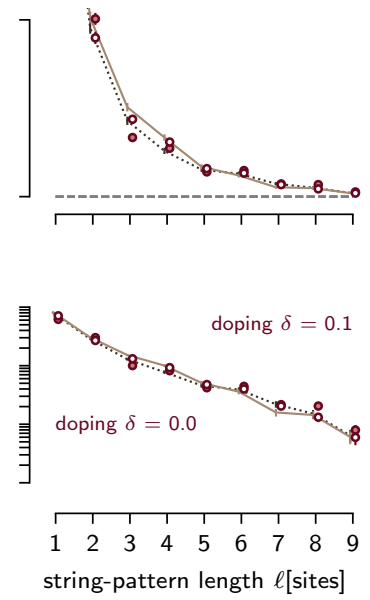

absolute difference

$p^{0.1}(\ell)-p_{s}^{0.1}(\ell)$

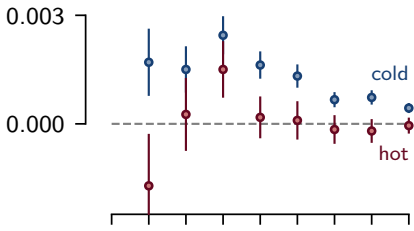

relative difference $p^{0.1}(\ell) / p_{s}^{0.1}(\ell)-1$

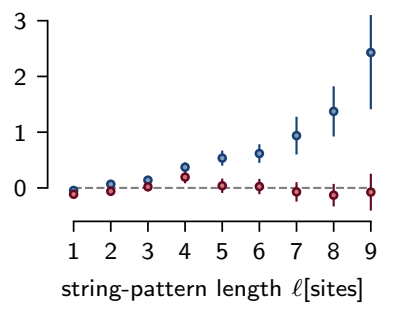

Figure 4.5: Measurement of string-pattern length histograms from site-resolved snapshots. a, Change in stringpattern length histograms upon doping, for the "cold" dataset. The observable is sensitive to doping in this regime, and simulated strings seem to fit the doped experimental result best. Points have been slightly offset horizontally for readability and histograms are normalized by the number of lattice sites analyzed. b, Same as a, but for the "hot" dataset. Here the observable is no longer sensitive to doping. c, Absolute and relative difference between doped and sprinkled-hole pattern-length histograms, highlighting temperature-dependent sensitivity.

\subsection{String-PATtern ObSERVABLES}

From the string patterns which have been extracted from each dataset, we examine three string-

pattern-based observables: the string-pattern length distribution, the string-pattern count, and the average string-pattern length. In line with our two key questions, for each of these observables I will discuss how useful they are and how they may be able to distinguish between $\pi$-flux states and geometric-string theory. 


\subsection{STRING-PATTERN LENGTH Distribution}

The first quantity we can examine is the distribution of string-pattern lengths for the string patterns found in each image, as seen in figure 4.5. In subfigure a, I show the distribution for our "cold" dataset of average temperature $T=0.65(4) \mathrm{J}$. The first point to note about the experimental data is that there is indeed an appreciable distribution $p^{0}(\ell)$ of string-pattern lengths $\ell$ detected at halffilling. Because there are no holes at half-filling which are not part of doublon-hole pairs, geometric strings are not predicted to exist at half-filling and this distribution reflects the deviation of a quantum antiferromagnet from our checkerboard approximation. It should therefore be considered as a baseline level.

As the sample is doped from half-filling to a doping $\delta$ of $10.1(8) \%$, the number of string patterns detected increases across the entire range of lengths. Intermediate-length string patterns increase most in absolute numbers, as seen in the linear-linear plot, but long-length string patterns increase most in relative numbers as seen in the linear-log plot.

Given that the string-pattern detection algorithm is sensitive to doping, we now compare to the three microscopic models to see which ones, if any, can explain the experimental result. For each of these models, we make predictions by producing artificial images and evaluating them with our string pattern detection algorithm, such that the detection is common to experiment and theoretical simulation. The results are shown in figure 4.5a. We find that the string-pattern length distribution for a doping of $10.1(8) \%$ agrees quantitatively with the experimental data. The measured signal does not simply result from the introduction of holes, as the sprinkled-hole simulation yields 
a string-pattern length distribution $p_{s}^{\delta}(\ell)$ which fails to explain the experimental results, revealing the nontrivial interplay of spin and charge degrees of freedom in the $2 \mathrm{D}$ doped Hubbard model. As for $\pi$-flux states at $10 \%$ doping, we find quantitative agreement with experiment at short pattern lengths, but a deficit at long lengths.

We repeat the measurements for a sample heated prior to lattice loading to investigate temperature effects. Figure 4.5b shows experimental data at half-filling as well as at 10.1(8)\% doping, along with the simulated prediction, averaged over samples at temperatures between $1.3(1) \mathrm{J}$ and 1.8(1) J. Notably, in contrast to colder temperatures there is no statistically significant deviation between the experimental data with and without hole doping; $p^{0.1}(\ell) \approx p^{0}(\ell)$. For these temperatures, thermal excitations may cause deviations from the reference checkerboard which are so large that the string patterns they create mask additional effects from doping. These deviations appear to set an upper bound on the density of detectable string patterns. As a result, I plot the pattern length distribution for high-temperature and half-filling as a reference for the cold temperature datasets in figure 4.5a.

Due to the baseline level $p^{0}(\ell)$, one ought to instead look at the absolute or relative difference between the pattern length histogram for the doped and undoped cases to examine the effect of doping. In addition, the sprinkled string-pattern length distribution $p_{s}^{\delta}(\ell)$ may be better to use for the undoped case as it is the density-adjusted counterpart of the experimental undoped distribution. Therefore, in figure $4.5 \mathrm{c}$ I plot the absolute difference $p^{0.1}(\ell)-p_{s}^{0.1}(\ell)$ and relative difference $p^{0.1}(\ell) / p_{s}^{0.1}(\ell)-1$ for both the hot and cold datasets. While the absolute difference does not recover the exact analytic string distribution, which can be attributed to the imperfect detection of the 


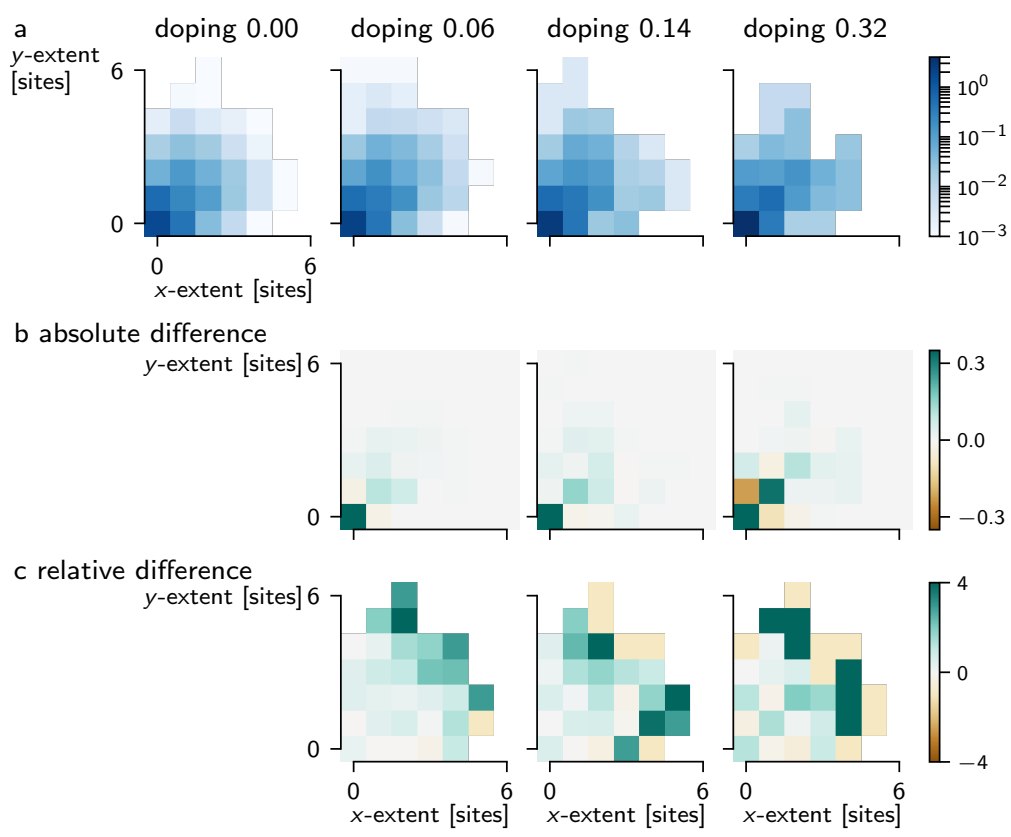

Figure 4.6: String-pattern shape distributions. a, Histogram of the spatial extent of detected string patterns in the two lattice directions ( $x$-extent and $y$-extent), for various doping values. They all appear to be isotropically distributed within statistical fluctuation, but perhaps with slightly more aspect ratios close to 1. b, Absolute difference between doped and half-filling histograms. The $(0,0)$ bins are saturated for visibility of the remainder of the plot. c, Relative difference. Some bins are saturated so that others are visible in the diverging colorbar. The large values of these saturated bins is likely due to statistical fluctuation.

pattern recognition algorithm, for cold temperatures it does find a qualitatively similar distribution.

Remarkably, at 10.0(8)\% doping we find over 3 times as many length-9 patterns as at half filling, reflecting the large impact of holes in an antiferromagnetic spin background.

While the string-pattern detection yields length distributions which seem reasonable and behave as one might expect with doping or an increase in temperature, we take a closer look at our results to scrutinize how meaningful they really are. First, we examine the shapes of the detected string patterns by considering their aspect ratios, that is, the $x$ - and $y$-extents of the smallest rectangle that contains the string pattern. Plotted in figure 4.6 are these aspect ratios histogrammed in a $2 \mathrm{D}$ histogram, normalized by the number of snapshots used (a), as well as the histogram difference from the half-filling case as an absolute difference (b) and relative difference (c). In both b and c, some of 


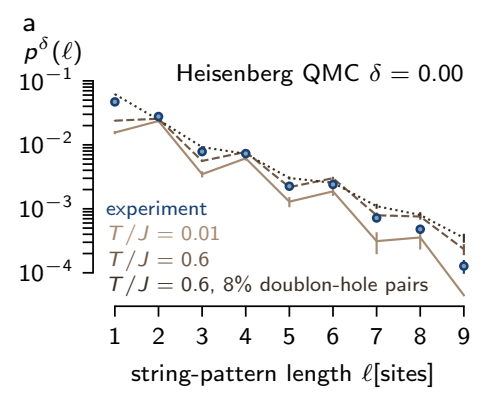

b

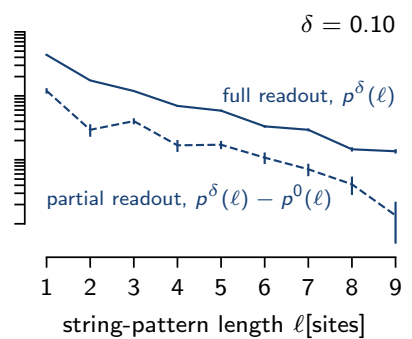

Figure 4.7: Contributions to the half-filling baseline and effect of partial spin readout. a, String-pattern length distribution for various Heisenberg QMC simulations at halffilling, compared to experiment. b, String pattern detection with simulated full spin readout and partial readout (with half-filling signal subtracted) using Heisenberg QMC simulation with added charge fluctuations and strings. The signals have the same shape but are offset due to the lower detection efficiency of partial spin readout.

the bins are saturated so that the other bins are visible in the diverging colorbar.

From these plots it appears that the detected string patterns are consistent with being isotropically distributed. This is in agreement with our calibration which fixes the tunneling in the $x$-and $y$-directions to be isotropic, and further suggests that the string-pattern detection does not bias for strings aligned in one direction or the other. Interestingly, it seems that there may be more string patterns with aspect ratios closer to I, as compared to very small or large aspect ratios. Additional investigations are necessary to quantify this asymmetry, but if it is statistically significant then string patterns with turns are more prevalent than straight string patterns and corrections to the linear string approximation in the geometric string theory would be necessary to account for this observation. An additional measurement to check how meaningful the string patterns are is to vary the tunnelling anisotropy and examine the subsequent anisotropy of string alignment, since a larger tunneling in one direction should lead to greater hole delocalization along that direction.

Second, we aim to understand the half-filling string-pattern length distribution more deeply. Apart from doublon-hole pairs, the Fermi-Hubbard model at half filling for $U \gg t$ can be ap- 
proximated by the Heisenberg model. We therefore examine the detected string-pattern length distribution from Heisenberg quantum Monte Carlo (QMC) simulation to better understand our experimental signal at half filling. For consistency, after simulating a 40 -site by 40 -site system with periodic boundary conditions, we cut out the same sample size and use the same readout and postselection schemes as in the experiment. We optionally add doublon-hole pairs into the simulated snapshots by converting neighboring sites with opposite spins into doublon-hole pairs with a probability given by $4 t^{2} / U^{2}$. We can also set the temperature of the Heisenberg model simulation.

Figure 4.7 a compares the string-pattern length distribution from the experiment at half filling with QMC simulations of the Heisenberg model. We first consider simulated snapshots at a temperature $T / J=0.01$ and no additional doublon-hole pairs. This low-temperature case allows us to examine the role of an $S U(2)$-symmetric quantum system. The resulting string-pattern length distribution already seems quite similar to that of experiment at half-filling, with the largest deviation for string patterns with an odd number of sites. In fact, the number of odd-length string patterns is noticably suppressed relative to even-length patterns. Because there are no doublon-hole pairs, these likely come from spin-exchange processes contibuting to a relative excess of string patterns of even length. The overall exponential-like behavior of string-pattern frequency with length, then, seems to be related to the $S U(2)$ symmetry of the system and non-maximal correlation strengths at long distances even at low temperatures.

The role of temperature is, as expected, also significant in contributing to the half-filling baseline. If the Heisenberg QMC temperature is increased to $0.6 J$ but we continue to omit doublonhole pairs, the resulting distribution matches experiment much more closely except for a continued 
stark deficit of length-I string patterns. The discrepancy is strongly reduced through the inclusion of doublon-hole pairs, suggesting that they play a non-negligible role in the half-filling baseline signal. At the same time, slightly more long string patterns are found in these higher-temperature Heisenberg QMC snapshots compared to experiment half-filling. Understanding this observation is likely not critical towards understanding the experimental half-filling baseline, but it may be explained by slight differences in the subtle relationship between staggered magnetization and correlation length. For example, at the same temperature the full counting statistics of the staggered magnetization shows a slightly broader distribution for the Heisenberg model relative to the Hubbard model ${ }^{20}$. The fixed postselection percentage would then yield images closer to the reference checkerboard for the Heisenberg model, suggesting fewer strings. However, if the correlation length is also longer for the Heisenberg model, then snapshots may contain larger areas that match or deviate from the reference checkerboard, yielding longer strings.

Finally, we investigate the role of incomplete spin information on our string-pattern length histograms. Indeed, in images taken in our experiment, we do not distinguish between holes, doublons, and the removed spin species. In a system with full readout, this distinction is available. In this case, the hole positions are known and the number of detected string patterns must correspond to the number of holes which are not in doublon-hole pairs, i.e. dopants. However, the detected distribution of string lengths can still be modified by overlaps between strings in the same way as in our experiment. We simulate full spin readout in QMC simulations of the Heisenberg model with simulated strings. Here too, the simulation is performed on a 40 -site by 40 -site system with periodic boundary conditions, from which a Io-site-diameter disk is cut out to match the experimental sys- 

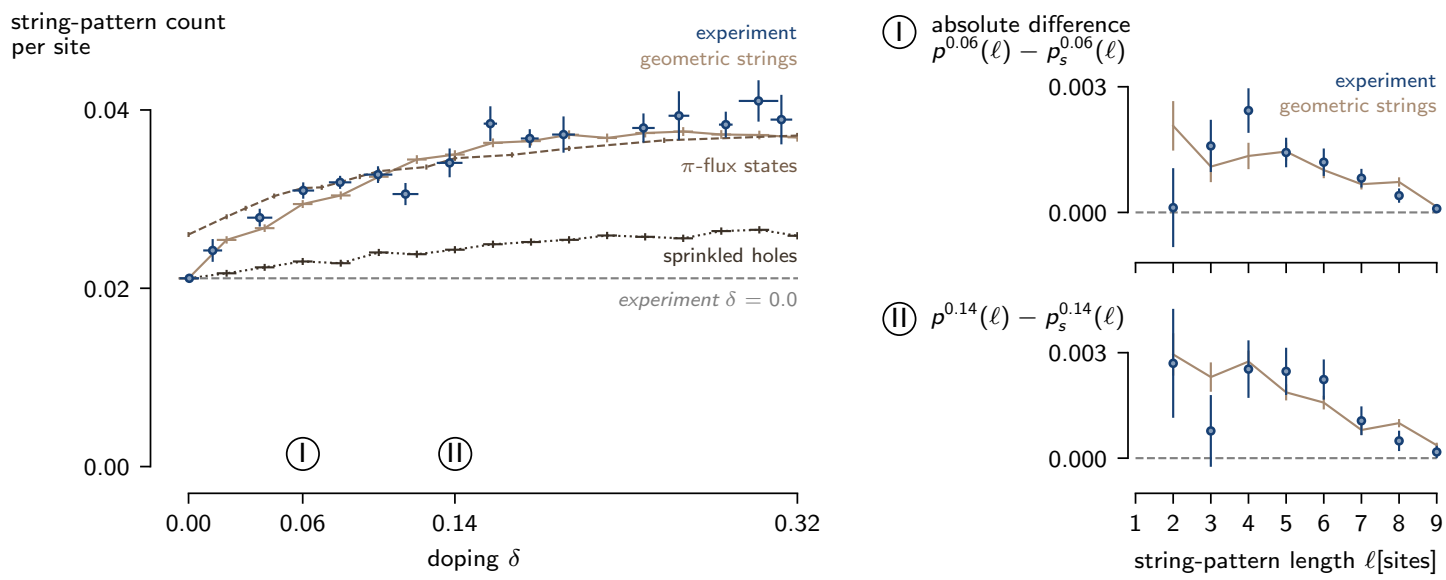

Figure 4.8: String-pattern count versus doping. a, Total number of string patterns exceeding length 2, normalized by the system size, as a function of doping. While the string model and sprinkled hole simulation both agree with experiment at half filling by construction, already at low doping the string model performs significantly better than sprinkled holes. The string model is quantitatively accurate across a larger doping range than for $\pi$-flux states, but both are in greater agreement with experiment than the sprinkled hole simulation. b, Absolute difference between experiment and sprinkled-hole string-pattern length histograms for $6.0(5) \%$ and $13.9(6) \%$ doping. While the absolute difference between experiment and sprinkled-hole pattern-length histograms increases with doping, the shape remains roughly invariant.

tem. Postselection is then done in the same way as in the experimental data analysis. In figure $4.7 \mathrm{~b} \mathrm{a}$ comparison of the detected string length distribution with and without full readout is shown, where the distribution obtained without full readout has the half-filling distribution subtracted. While the signal with full readout is a factor of about five higher, the relative distribution of the detected string-pattern lengths remains the same.

\subsubsection{STRING-PATTERN COUNT}

Focusing on the cold dataset, in figure 4.8 we now examine the relationship between doping and the number of detected string patterns. In this string-pattern count we omit patterns of I or 2 sites to avoid contributions from quantum fluctuations such as doublon-hole pairs or spin-exchange 
processes. The number of patterns increases with doping and saturates at about $16 \%$ doping. This saturation is consistent with a high density of strings where they begin to overlap or lie adjacent to one another, scrambling spin order such that pattern detection becomes insensitive to additional strings. The continued agreement between geometric strings and experiment in both the stringpattern count and the absolute difference $p^{\delta}(\ell)-p_{s}^{\delta}(\ell)$, shown for dopings $6.0(5) \%$ and $13.9(6) \%$, suggests that the increase in number of geometric strings is sufficient to explain the experimental data.

The experimental string-pattern count is significantly larger than that of the sprinkled-hole simulation; nonetheless, there is an increase in detected string patterns simply due to the introduction of holes. Because of this effect, we use the sprinkled hole length distributions $p_{s}^{\delta}(l)$ rather than $p^{0}(l)$ for the absolute and relative differences in figures 4.5 and 4.8. The string-pattern count from $\pi$-flux states shows significantly better agreement with experimental data than sprinkled holes, exhibiting only a slight excess of string patterns at low doping and a deficit at high doping. The largest deviations occur at low doping, which may be related to the absence of long-range order at zero temperature in $\pi$-flux states at half-filling.

For low doping, we can also fit the data of figure $4.8 \mathrm{a}$ to a line and estimate a string detection efficiency for strings of lengths greater than two sites. We find a slope of $1.7(2) \times 10^{-3}$ string patterns per site per percent doping for doping up to $6 \%$. The analytically calculated string length distribution for a temperature of $0.6 \mathrm{~J}$ predicts that $65 \%$ of string states have length greater than two sites, giving an approximate detection efficiency of $25(2) \%$.

We use the string-pattern count to look more closely at our postselection and verify that we do 


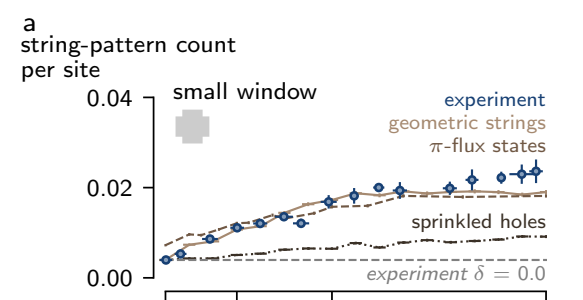

b

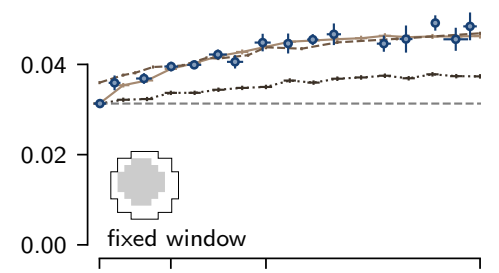

C

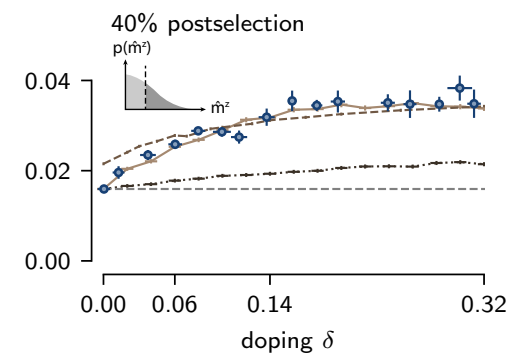

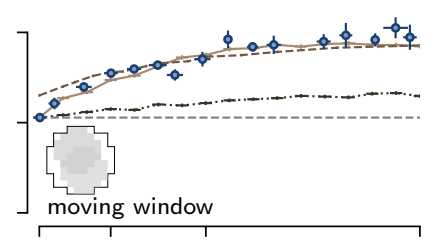
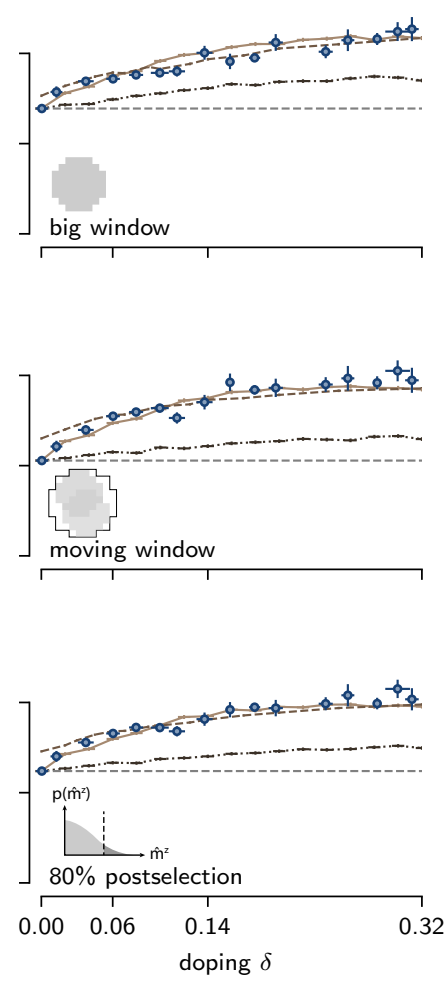

Figure 4.9: Effect of string count on post-selection. All parameters not mentioned are kept fixed as in the procedure outlined in section 4.3.1. In all cases, we see that the qualitative features seen in figure 4.8 are maintained. a, Using a differentsize window for the analysis region, either 5 or 8 sites in diameter. b, Fixing the window position to the center of the system, compared to scanning the window position to maximize the staggered magnetization. c, Varying the percentage of data kept when postselecting on the staggered magnetization, either $40 \%$ or $80 \%$.

not unintentionally introduce biases through our postselection choices. Figure 4.9 depicts three types of postselection and possible variations on what we have chosen for each type. To begin, we choose an analysis region which is smaller than the entire Io-site-diameter system; our postselection region is chosen to be 7 sites in diameter according to the antiferromagnetic correlation length at half filling. We vary the window to a smaller circular region of 5 sites in diameter, or to a larger circular region of 8 sites in diameter, and find that the qualitative dependence of the string count on doping remains the same. The baseline string count at half filling increases for larger postselection regions as the region becomes larger than the correlation length and the deviation from the reference checkerboard increases. 
We also consider the effect of moving the window to achieve the highest values of the staggered magnetization. We find that fixing the window to the center of the system while keeping a postselection threshold of $60 \%$ greatly increases the number of string patterns found at half filling relative to the additional number of patterns found upon doping the system. This is due to a greater average deviation from the reference checkerboard. In principle, we could achieve a better signal to noise by postselecting more strongly on which images we use, at the cost of increased statistical fluctuation. While the half-filling value changes, the estimated detection efficiency does not change statistically significantly, indicating robustness of the detection algorithm to this effect.

Finally, the fraction of images kept in the post-selection process can be varied. We choose to keep the top $60 \%$ of images in an effort to capture the tail of staggered magnetization histogram, while maintaining a reasonably high number of images. Upon changing the postselection to $40 \%$ or $80 \%$, we find fewer or more string patterns at half-filling, respectively. However, the slope of the stringpattern count as a function of doping in the low-doping regime does not change statistically significantly.

In addition to examining postselection with the string-pattern count, we examine the analytic string length distribution as predicted by geometric-string theory. In figure 4.Io we vary the predicted distribution and examing how the resulting detected string-pattern length distribution changes. Changing the temperature for Boltzmann sampling of the string states yields a worse agreement with the experimental result, as does changing the participation ratio of holes in strings by only moving a fraction of the holes which have been randomly placed. We also alternatively select only strings of a given length and find the best agreement for strings of length 4 ; this is close to the 
a

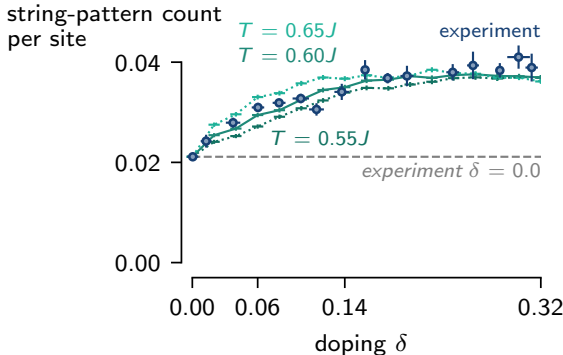

b

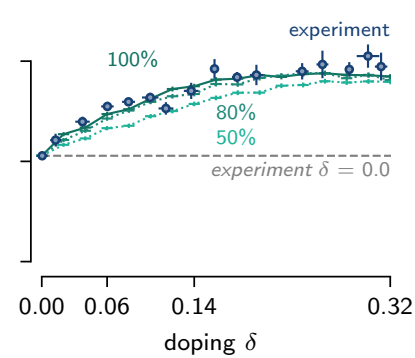

c

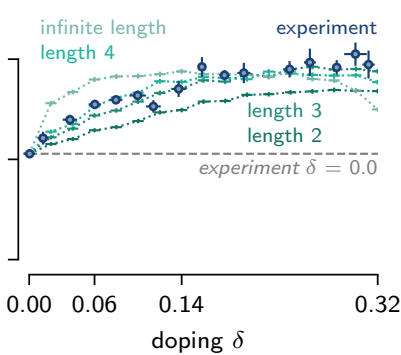

Figure 4.10: Effect of analytic string length distribution on measured string-pattern count. a, Comparison of experimentally measured string-pattern count and simulated geometric strings, for analytic string length distributions corresponding to temperatures $T / J$ of $0.55,0.60$ (as in all other figures), and 0.65 . The $T / J=0.60$ distribution matches experiment best. b, Same, but varying the percentage of simulated holes which are then propagated to simulate strings. The best agreement occurs when all holes are part of strings. c, Same, but for simulated strings which are of all of a single length, ranging from 2 sites to the infinite-site limit, instead of sampled from the analytic distribution.

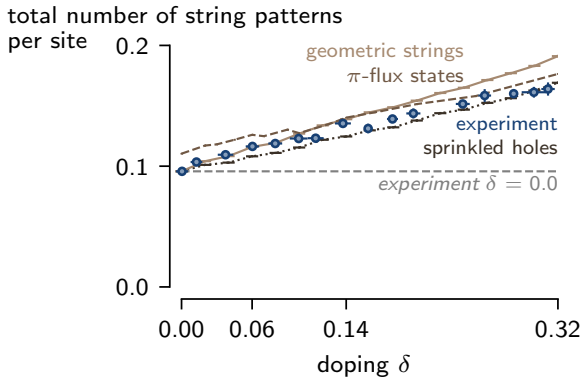

Figure 4.11: Total number of string patterns detected versus doping. String-pattern count versus doping, as in figure 4.8 , but including all string-pattern lengths in the count. The agreement between all simulations and experiment suggests that this quantity may be trivially dependent on doping.

average string length at $T=0.6 J$ of 4.2 . From these results we conclude that perturbations to

the analytic string length histogram are unlikely to improve agreement with the experimental mea-

surement. We note that the decrease in string count at very high doping for infinite-length strings is likely an artifact from simulation, caused by difficulties in propagating holes large distances without overlapping with other holes or backtracking.

In figure 4.II we include all string-pattern lengths in computing the string-pattern count, rather than omitting string patterns of length one or two sites. We find that all simulations show similar agreement with experimental data, and all string-pattern counts increase linearly with doping. This 
linear behavior indicates that this quantity may simply reflect the doping level, and is not a useful observable. Interestingly, however, the slopes of all simulations differ slightly, and the experimental data seems to show a slight saturation effect after a doping of about $6 \%$. At low to intermediate doping, where we are sensitive to the string-pattern count, the increase in the number of string patterns of all lengths grows at a rate almost identical to the geometric-string theory. However, in the overdoped regime the slope for the experimental data decreases slightly and the number of string patterns becomes closer to that of $\pi$-flux states or even the sprinkled holes. This effect is not completely understood and may warrant additional study.

Finally, we can better understand the role of temperature in string-pattern detection by observing how the string-pattern count varies with temperature at fixed doping. For $10 \%$ doping, we plot the difference between the experiment and sprinkled-hole string-pattern counts, see figure 4.I2, with the individual values plotted in the inset. At our lowest temperatures, the difference is at its greatest. This high sensitivity is consistent with the greatest spin ordering for the parent antiferromagnet at low temperatures, accompanied by a relatively large string count from the experimental data. The difference decreases steadily with increasing temperature, predominantly due to the increase in the sprinkled-hole string count as a consequence of decreased spin ordering in the parent antiferromagnet, vanishing around $T=J$. Therefore, we conclude that the string observables are only sensitive at low temperature and low to intermediate doping, much like the staggered magnetization order parameter or spin-spin correlations. 


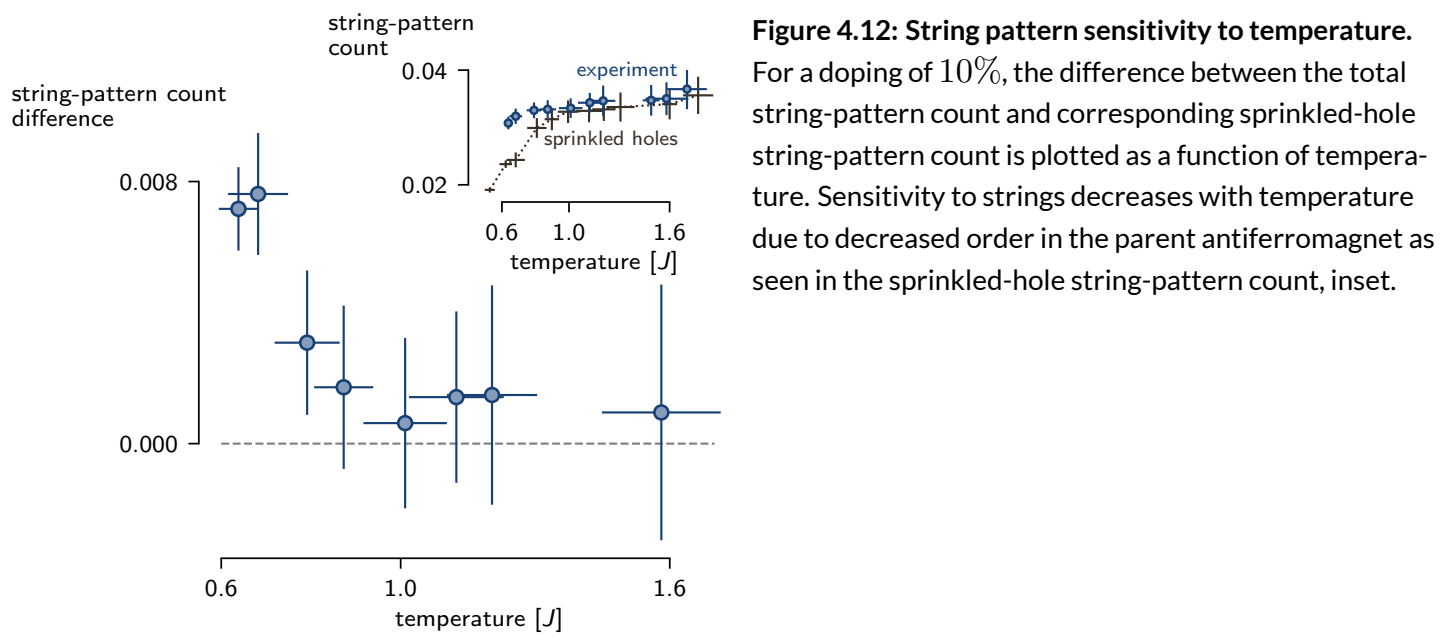

\subsubsection{Average string-Pattern length}

The average string-pattern length quantifies the size of the region around the hole where the spin pattern is distorted by the string, see figure 4.I3. The observed values are comparatively small, influenced by the large contributions from quantum fluctuations at half-filling. The average stringpattern length does not change dramatically with doping, consistent with independent patterns; however at larger dopings we observe a slight decrease in average length that coincides with the observed saturation in the string count. This behavior is captured by the geometric-string model for low and intermediate doping. At high doping, the theory exhibits shorter average string lengths than the experiment, which may be due to high-string-density effects such as string-string interactions which are not included in the theory.

We compare these results to a dataset where geometric strings are not expected to occur. This dataset consists of experimental images taken at various temperatures at half filling ("temperature datasets”) with sprinkled holes to match each desired doping level. More specifically, for each desired 


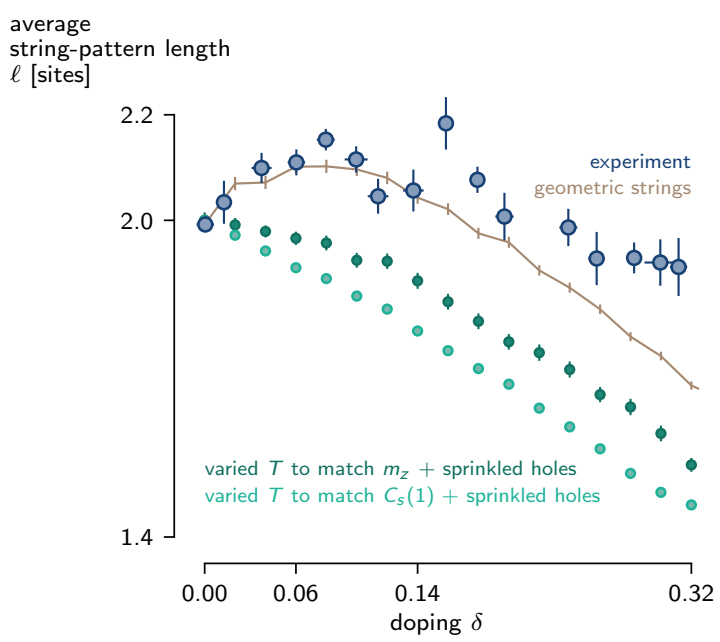

Figure 4.13: Average string-pattern length versus doping. Doped antiferromagnets exhibit longer-length string patterns compared to heated antiferromagnets, even when the staggered magnetization or nearest-neighbor spin correlator is equal and holes are sprinkled in to equate doping levels.

doping value we randomly place holes into all temperature datasets to artificially achieve the doping value for every temperature dataset. We then extract the staggered magnetization and average string length of each dataset, obtaining the relationship between these two quantities. We perform a linear fit to obtain $\bar{l}^{\delta}\left(\left\langle\hat{m}^{z}\right\rangle\right)$, the average string length at a given doping as a function of average staggered magnetization, for the temperature datasets.

To determine which value of $\left\langle\hat{m}^{z}\right\rangle$ to use in this function, we use the low-temperature experimental datasets taken at low temperature and various dopings (“doping datasets”). As the dependence of the measured staggered magnetization on doping is non-linear for these datasets, we perform a linear fit of the closest five data points for each doping value to obtain a reliable estimate of $\left\langle\hat{m}^{z}\right\rangle$. This value is then used to determine the average string length for the temperature datasets. The entire process is repeated for each doping value to obtain the data in figure 4.I3 (dark teal circles).

This procedure allows us to directly compare the experimental data at finite doping to a scenario 
where the staggered magnetizations are similar and the same number of doped holes are present but no geometric strings are included. The error bar for the predicted average string length is obtained by combining the measurement error of $\left\langle\hat{m}^{z}\right\rangle$ with the error of the linear fits weighted by the standard deviation of the measured quantities. As a cross-verification, we have applied the same procedure except for choosing the "effective" temperature by matching the value of the nearestneighbor spin correlator. With this method we found the same qualitative behavior, see figure 4.I3 (light teal circles).

To summarize, temperatures are chosen to match the measured staggered magnetization and obtain a dataset that captures the observed loss of antiferromagnetic order. Notably, the average string-pattern length reveals that this loss through heating occurs in a fundamentally different way than through doping. For all nonzero doping, the temperature-based dataset exhibits shorter average string-pattern lengths than the experimentally measured doping dataset, across all doping values. This does not seem to depend on how the dataset is parametrized.

At this point, we have introduced all of the string-pattern-based observables and seen that they offer a new way to compare experimental results to microscopic theoretic predictions. We detect signatures of string patterns that require sufficiently low temperatures and change significantly when the system is doped. Further, these patterns are differentiated from those that appear when heating the system. At temperatures just below the super-exchange energy and across a wide range of doping values, the presence and behavior of these patterns upon changing system parameters favors the geometric-string and $\pi$-flux models over sprinkled holes, with slightly stronger agreement for geometric strings. 


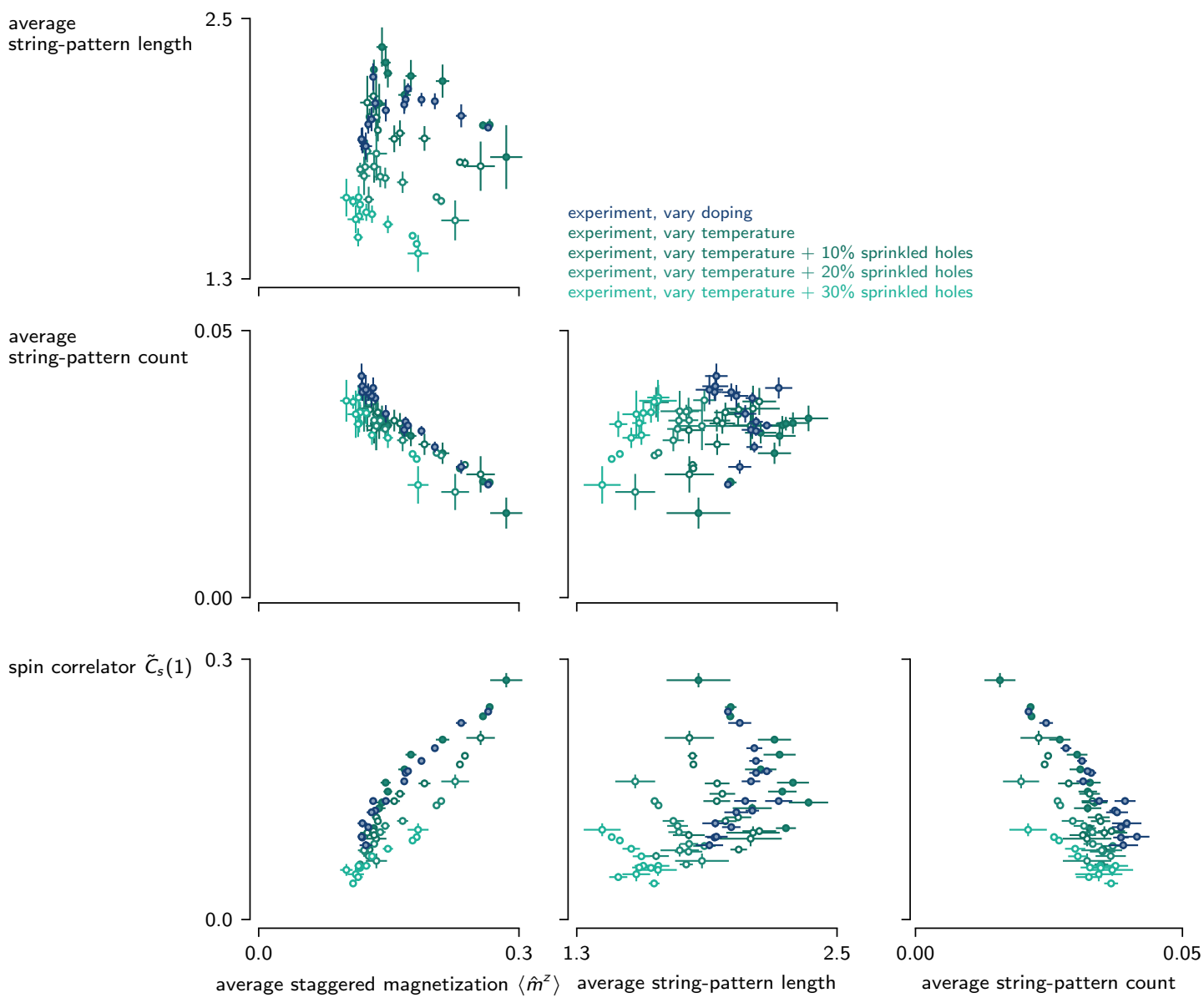

Figure 4.14: Multi-parameter comparison of doped and heated antiferromagnets. We plot pairwise the average staggered magnetization, sign-corrected nearest-neighbor spin correlator, average string pattern length, and average string-pattern count for both doped and heated antiferromagnets, as well as density-adjusted heated antiferromagnets. By examining the values which can be obtained by each dataset, we identify regimes attainable by doping but not heating, or vice-versa. 
We can take a even deeper look at how string-pattern based observables may be able to capture more information beyond what can be described with conventional spin observables. Indeed, in figure 4.13 we see that there are certain pairs of values for the average string length and average staggered magnetization (or nearest-neighbor spin correlator) which can be achieved through doping an antiferromagnet, but cannot be achieved by heating it. We extend this analysis by plotting the average staggered magnetization $\left\langle\hat{m}^{z}\right\rangle$, sign-corrected nearest-neighbor spin correlator $\tilde{C}_{s}(1)$, average string-pattern length, and average string-pattern count pairwise for all possible combinations, as shown in figure 4.I4. We begin by including two experimental datasets, one being our "cold" dataset where doping is varied from half-filling to approximately $32 \%$, and the other being a half-filling dataset where the temperature is varied instead, from our coldest temperatures of $0.50(4) \mathrm{J}$ up to $1.8(1) J$

Beginning in the lower left corner of figure 4.I4, we find that the set of $\left(\left\langle\hat{m}^{z}\right\rangle, \tilde{C}_{s}(1)\right)$ values which can be obtained through doping almost exactly match those which can be obtained through heating. Given that the average staggered magnetization can be derived from the spin correlation function, this result is not entirely surprising; however it does show a very close relationship between the value of the nearest-neighbor correlator and spin correlations at longer distances.

Moving to the other observables, we see that the set of values covered by doping does not directly overlap with the set covered by varying the temperature. In other words, there appear to be values of the string-pattern-based observables which can differentiate between these two types of loss of antiferromagnetic order. For example, consider the average string-pattern count and the nearestneighbor spin correlator (lower right corner of figure 4.I4). Upon doping the antiferromagnet, val- 
ues of the average string-pattern count of greater than 0.035 per site can be achieved. By contrast, this is not possible by heating the antiferromagnet. For temperatures outside the range of temperatures considered, the trend of points suggests a saturation or even a slight decrease in the average string-pattern count compared to the values shown. We additionally plot datasets where some fixed number of holes is sprinkled into the snapshots taken for the temperature dataset, to see if adjusting for the density may be sufficient to cover the same set of values. In the case of the average stringpattern count, it does increase slightly, but at the same time the value of the nearest-neighbor spin correlator decreases sharply. As a result, this artificial density correction is not sufficient to achieve the same high values as seen by doping the system.

We also find values of string-pattern-based observables which can be achieved by heating an antiferromagnet, which cannot be achieved by doping it. Take the upper left corner of figure 4.I4, which plots the average string-pattern length versus the average staggered magnetization. Here the average string-pattern length reaches a maximum length of about 2.2 sites, as seen also in figure 4.I3. Yet when the temperature is increased instead, higher values of the average string-pattern length can be achieved for the same average staggered magnetization. These results suggest different underlying microscopic mechanisms for the loss of antiferromagnetic order through doping or heating. Crucially, one cannot see this through considering only the spin-spin correlator or staggered magnetization, but can upon including spin-pattern-based observables. And that's pretty cool. 

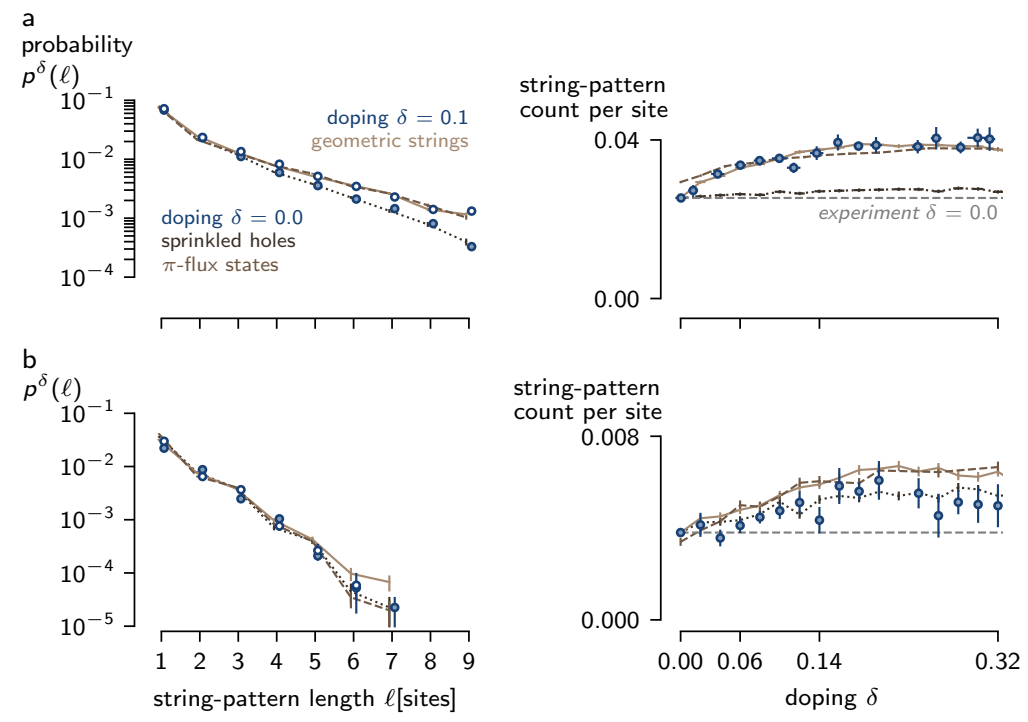

Figure 4.15: Alternate string detection schemes. a, The stringpattern length histogram and string-pattern count produced with the simplified difference pattern extraction algorithm. Apart from an increase in values of the string-pattern-based observables at half-filling, the results are similar to the main detection algorithm. b, Same quantities, but produced with the happiness method, are also sensitive to doping but may be too noisy to be useful.

\subsection{Alternate Detection schemes}

There are several possible algorithms which can be used to detect patterns based on the geometricstring theory, and many more which can be used to detect any type of generic pattern. Here I discuss two alternate algorithms for string-like patterns. We find that these algorithms are comparable in performance to the preferred detection algorithm at the focus of this chapter, and determine our algorithm of choice based on a balance of simplicity and making use of all information available.

\subsection{SiMPLIFIED DIFFERENCE METHOD}

The most straightforward way to detect string patterns is to simply count the continuously connected sites that deviate from the classical checkerboard pattern. As opposed to the algorithm we have been using thus far, not every object identified as a string pattern in this way can actually be a geometric string. For example, it is possible that both endpoints as well as the sites surrounding 
them are occupied such that there cannot be a hole at either end. Moreover, the shape of the object may not be consistent with a non-branching string pattern. However, one can argue that these inaccuracies mainly occur at high temperature or high doping values when perturbations and strings start to overlap.

In figure 4.I5a, the string-pattern length distribution and string-pattern count are shown under the simplified string detection algorithm. At a doping of about $10 \%$, both $\pi$-flux states and geometric strings seem to quantitatively match the experimentally measured string-pattern length distribution well. The total string count versus doping looks qualitatively similar compared to that of the preferred algorithm. The detection efficiency remains roughly the same as before, while the half-filling baseline is slightly larger.

\subsubsection{Happiness Method}

In the dilute string regime, where string states do not overlap or lie adjacent to one another, one can search for string patterns by also requiring that sites immediately surrounding the string pattern maintain antiferromagntic order. Note that this requirement also omits string states which have segments that lie adjacent to each other, for example string patterns containing a tight "U"-shape. This method is also susceptible to identifying string patterns caused by doublon-hole pairs, spinexchange processes, and projective measurement. However, as these effects will introduce deviations from antiferromagnetic order, this is perhaps the most conservative approach to finding string patterns.

This algorithm characterizes nearby order by labeling each site with the number of anti-aligned 
bonds it has with its nearest neighbors, termed the "happiness" of that site, for images with one spin species removed. For example, sites in a classical antiferromagnet would all be labelled with happiness 4, while a ferromagnet would have sites with happiness $\mathrm{o}$. As a hole moves through an antiferromagnet, sites which previously had happiness 4 will exhibit reduced happiness. Depending on the length of the string, sites within a string will have specific happiness values. Based on this, the algorithm takes images with one spin state removed and for each image, begins by storing all sites which could be the beginning of a string. For each candidate string beginning, it sees if there is a neighboring site that could be the next site in the string, given the happiness and spin occupation of that site. This process continues until the string cannot be propagated any further, at which point the algorithm searches for a neighboring site which could be the end of the string.

Figure 4.I5b shows the string-pattern length distribution and string-pattern count under the happiness string detection algorithm instead. Note that the signal to noise ratio is significantly lower and the absolute signal itself is lower by almost an order of magnitude. This is not surprising, especially given that quantum fluctuations and projection noise do contribute considerably to measurement and reduce the sensitivity of string patterns to string states. Here the experimental result seems to best match the sprinkled holes simulations, however uncertainties are large and this is highly inconsistent with all other results, requiring further investigation. 

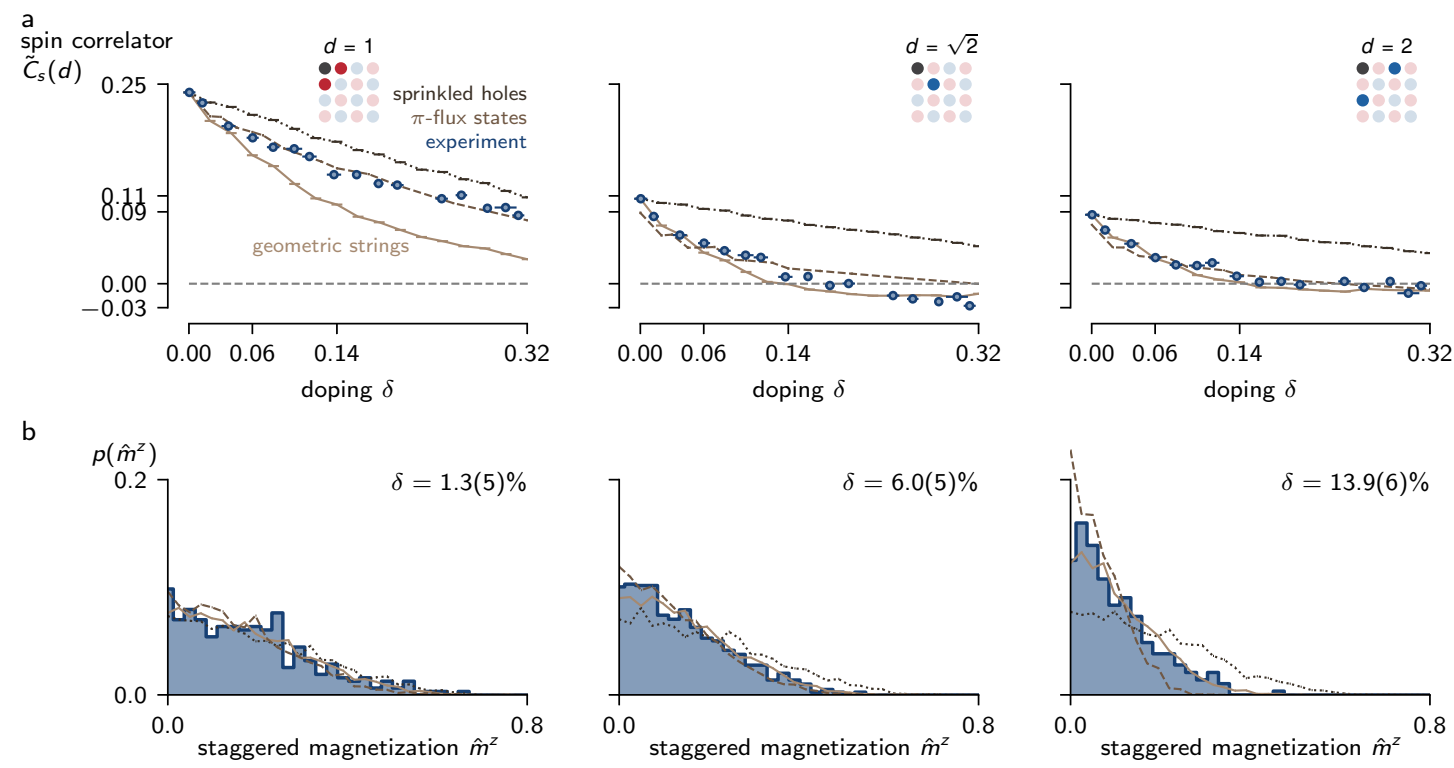

Figure 4.16: Spin correlations and staggered magnetization. a, Decay of nearest-neighbor (left), diagonal next-nearestneighbor (center), and straight nearest-neighbor (right) spin-spin correlation functions upon doping. While the $\pi$-flux theory most quantitatively explains $\tilde{C}_{s}(1)$, only the string model captures the sign change of $\tilde{C}_{s}(\sqrt{2})$. In all three cases, sprinkled holes overestimate the spin correlations. b, Full counting statistics of the staggered magnetization for doping values of $1.3(5) \%$ (left), $6.0(5) \%$ (center), and $13.9(6) \%$ (right). Here both $\pi$-flux states and geometric strings show reasonable agreement, while sprinkled holes do not.

\subsection{Conventional SPIN OBSERVABLES}

An accurate microscopic framework for the Hubbard model should also be able to predict more conventional observables such as two-point correlation functions, which have been used with quantum gas microscopes to quantify spin and charge $\operatorname{order}^{19,76,87,62}$. To that end, we measure the signcorrected spin-spin correlation function $\tilde{C}_{s}(d)$ for displacements $|\mathrm{d}|=d$, averaged over all sites in the system and all experimental realizations, by measuring charge correlations in experimental realizations with and without spin removal ${ }^{19}$. We include a sign correction such that positive correlator values indicate antiferromagnetic ordering. Figure 4.16a shows the change in the nearest 
neighbor, diagonal next-nearest neighbor, and straight next-nearest neighbor sign-corrected spin correlators $\left(\tilde{C}_{s}(1), \tilde{C}_{s}(\sqrt{2})\right.$, and $\tilde{C}_{s}(2)$, respectively) as a function of doping at $T=0.65(4) J$. At half-filling, $\tilde{C}_{s}(1)$ is substantially larger than both $\tilde{C}_{s}(\sqrt{2})$ and $\tilde{C}_{s}(2)$ due to a strong admixture of spin singlets on adjacent sites $^{\mathrm{I} 34}$. As the system is doped, all correlators exhibit a reduction in magnitude. While $\tilde{C}_{s}(1)$ remains positive for all experimentally-realized doping values, $\tilde{C}_{s}(\sqrt{2})$ exhibits a statisticially significant sign change around $20 \%$ doping. These features have been observed in experiment ${ }^{19,87}$ and numerics ${ }^{87}$, and are good benchmarks for the evaluation of theoretical models.

We make predictions for spin correlations from ensembles of non-post-selected images with sprinkled holes, geometric strings, or $\pi$-flux states. At half filling, sprinkled holes and the string model make the same predictions as the experimental half-filling data by construction. Away from half filling, sprinkled holes underestimate the decrease of the correlators since it fails to account for the disruption of antiferromagnetic order as the system is doped. In contrast, beginning at intermediate doping values the string model overestimates the decrease of $\tilde{C}_{s}(1)$, which could stem from backaction of the background state after string state formation. However, it explains the decrease of $\tilde{C}_{s}(\sqrt{2})$ and $\tilde{C}_{s}(2)$ on a quantitative level. The $\pi$-flux model performs well and accurately predicts $\tilde{C}_{s}(1)$ and $\tilde{C}_{s}(2)$ far from half-filling, but fails to predict the sign change of $\tilde{C}_{s}(\sqrt{2})$ at intermediate doping, even when the fitted temperature is varied. The sign change of $\tilde{C}_{s}(\sqrt{2})$ is an interesting qualitative feature that is predicted and can be explained by the string model. As a direct result of spins being displaced by one site when a string passes through, $\tilde{C}_{s}(1)$ is mixed into $\tilde{C}_{s}(\sqrt{2})$. Because $\tilde{C}_{s}(1)$ reflects opposite spin alignment from $\tilde{C}_{s}(\sqrt{2})$, this mixing results in a sign change once the contribution of $\tilde{C}_{s}(1)$ exceeds that of the original correlation strength at some critical doping. 
Cold atom experiments also provide access to full-counting statistics (FCS) due to their ability to project and measure an entire quantum system at once ${ }^{20}$. We measure the FCS of the staggered magnetization operator $\hat{m}^{z}$ across all experimental realizations as we dope the system, see figure4.I6b. As expected, the staggered magnetization distribution narrows, reflecting the finite-size crossover from the antiferromagnetically ordered phase ${ }^{20}$. The sprinkled-hole simulation does not exhibit a major change in the distribution as the system is doped, as it fails to account for the disruptive effect of holes on the antiferromagnetic order. By contrast, both $\pi$-flux states and geometric strings demonstrate reasonable agreement with the experimentally measured distribution function across all dopings. Across all observables considered, both of these theories perform quite well, especially in comparison to the sprinkled holes simulation. However, we find the sign change of $\tilde{C}_{s}(\sqrt{2})$ to be a key qualitative feature which is only captured by geometric strings.

\subsection{Hole-hole correlations}

All observables studied in this chapter thus far have focused on the spin sector of the Hubbard model. Now I examine correlations in the charge sector. At sufficiently low temperatures, one may expect signatures of pairing ${ }^{8,135}$ or stripe phases ${ }^{\mathrm{r} 36, \mathrm{r} 08}$, which lead to hole bunching. On the other hand, anti-correlations of the holes, as observed previously at elevated temperatures ${ }^{87}$, are expected in the strongly correlated metallic regime of the Hubbard model. The transition between these two regimes in the Hubbard model phase diagram is not yet fully understood, however the currently accessible experimental regime allows us to place new bounds on where this transition can occur. 
We continue to compare to predictions of $\pi$-flux states, but do not compare to predictions of the geometric string theory because it approximates that charges are uncorrelated. Rather, because each string is associated with a single hole, correlation functions of holes can reveal possible interactions and correlations between geometric strings, should they exist.

In our experiment, doubly-occupied sites appear as empty when imaged and the exact hole correlation is not directly accessible; rather, we measure "anti-moment" correlations $C_{h}(|\mathrm{~d}|)$ at a distance |d| which include contributions from doublon-doublon and doublon-hole correlations:

$$
C_{h}(|\mathrm{~d}|) \equiv\left(\left\langle\left(1-\hat{n}_{s, \mathrm{i}}\right)\left(1-\hat{n}_{s, \mathrm{i}+\mathrm{d}}\right)\right\rangle-\left\langle\left(1-\hat{n}_{s, \mathrm{i}}\right)\right\rangle\left\langle\left(1-\hat{n}_{s, \mathrm{i}+\mathrm{d}}\right)\right\rangle\right)
$$

where $\hat{n}_{s, \mathrm{i}}$ is the single particle occupation on site $\mathrm{i}$. Note that this correlator is identical to the moment correlator. At half-filling, numerics indicate positive anti-moment correlations at the percent level for nearest neighbors, dominated by positive doublon-hole correlations ${ }^{87}$. Doublon-hole pairs beyond nearest-neighbors become increasingly unlikely; therefore, to avoid the effects of doublonhole pairs we focus on correlations at distances greater than one site. We find the nearest-neighbor anti-moment correlator at half-filling to be weaker than predicted according to numerics, which may result from imperfect imaging fidelity. However, this effect only weakens the magnitude of the antimoment correlators measured; as such in this section we focus on qualitative conclusions from the experimental data.

Figure 4.I7a shows the anti-moment correlation function across several doping values from the half-filling to the overdoped regime at a temperature $T=0.65(4) \mathrm{J}$. While holes appear uncor- 


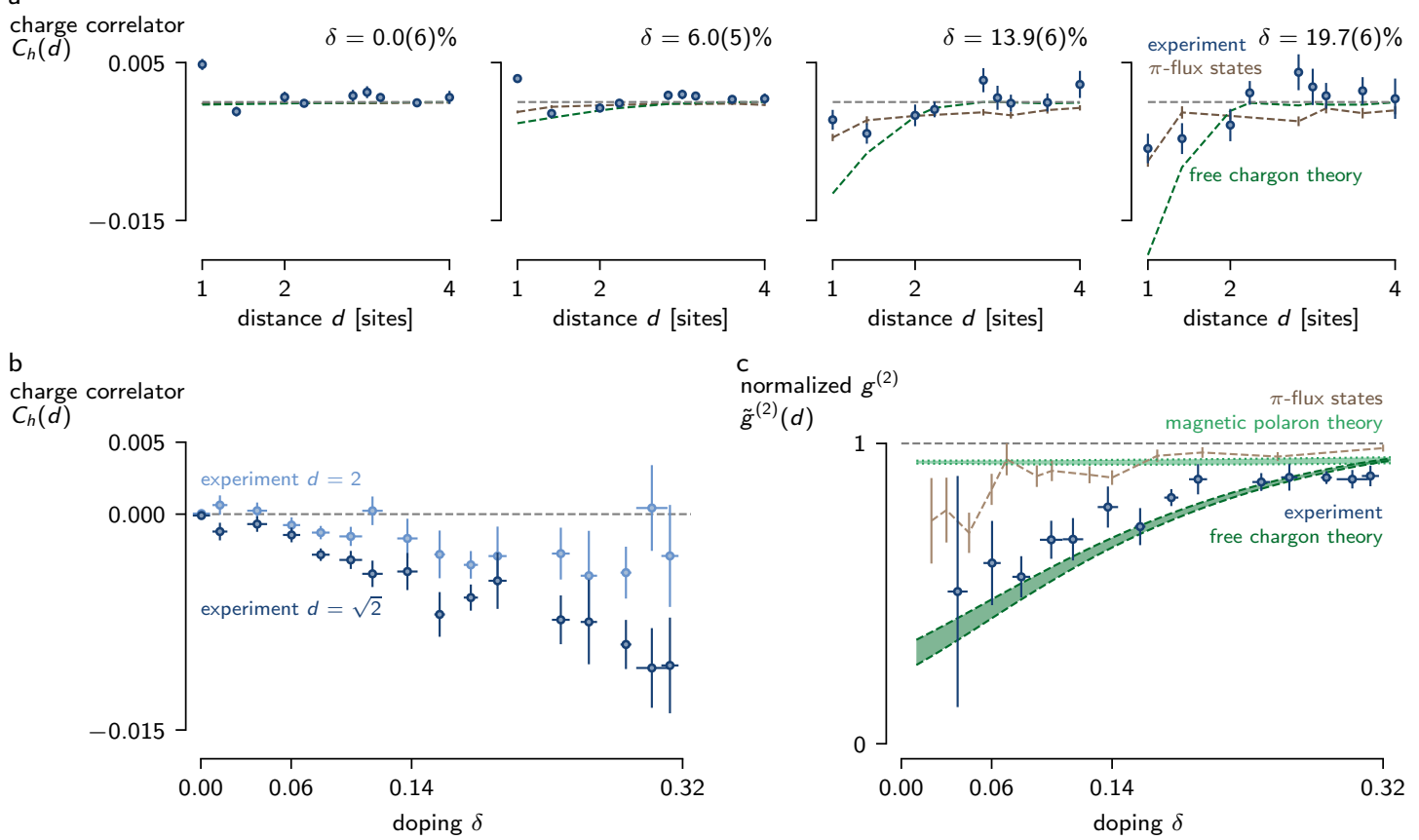

Figure 4.17: Observation of hole anti-bunching. a, Anti-moment correlation function for various doping values. The correlation functions show a growth of hole anti-bunching with doping out to a distance of $d=2$. $\mathbf{b}$, Diagonal nextnearest neighbor and straight next-nearest neighbor anti-moment correlators versus doping. At both distances negative correlations grow with doping. c, Normalized anti-moment correlator at $d=\sqrt{2}$ versus doping. The experimental result cannot be explained by the $\pi$-flux or a point-like magnetic polaron theory, but instead matches a free fermionic chargon theory.

related close to half-filling, at larger doping qualitatively different behavior appears. We find statistically significant anti-moment anticorrelations out to distances greater than two sites, reflecting hole-hole repulsion in this regime. Microscopically, such repulsive interactions can arise from the existence of a low-lying bound state of two holes ${ }^{36}$. Here we do not consider geometric-string theory or sprinkled holes because both introduce uncorrelated holes by construction. Additionally, in the comparison to $\pi$-flux states we do not include doublon-hole pairs to avoid unintended artifacts in the anti-moment correlator. For reference, we plot the predicted hole-hole correlation function for a 
phenomenological model of spinless fermionic chargons with nearest-neighbor hopping of strength $t$ and a temperature of $0.6 J^{137}$. This model is motivated by the possibility that spinon-chargon pairs unbind to realize a deconfined phase chargons. For simplicity we consider free fermions, where strong anticorrelations result from Pauli repulsion, but qualitatively similar behavior is expected for bosonic chargons with hard-core interactions. More informed theoretical work has also proposed the possibility of a non-trivial metallic state of chargons ${ }^{\mathrm{I} 37}$. We find that both $\pi$-flux states and free fermionic chargons qualitatively describe the experimental result.

The emergence of this repelling behavior can be characterized by plotting the anti-moment correlation as a function of doping for $d=\sqrt{2}$ and $d=2$, see figure4.I7 b. Beyond the intermediate doping regime, negative correlations appear at distances of $\sqrt{2}$ and 2 , suggesting a growth of holehole repulsion with doping. Furthermore, the presence of anti-moment correlations between sites of differing sub-lattices at $d=1$ evidences against holes tunneling preferentially between sites of one sub-lattice, as predicted by theories of point-like magnetic polarons with a dispersion minimum at $(\pi / 2, \pi / 2)$ in the Brillouin zone $e^{\mathrm{I} 38, \mathrm{r} 39, \mathrm{r} 23, \mathrm{r} 4 \mathrm{O}}$.

Finally, we plot a normalized $g^{(2)}(d=\sqrt{2})$ to account for the difference between doped holes and holes in doublon-hole pairs and quantify the relative fraction of doped holes that are anticorrelated:

$$
\tilde{g}^{(2)}(|\mathrm{d}|) \equiv \frac{C_{h}(\mathrm{~d})}{\delta^{2}}+1
$$

for doping $\delta$, see figure4.I7c. This rescaling allows direct comparison to the $g^{(2)}$ function for theories without doublon-hole pairs. Because the number of free holes is so small for doping below 
$5 \%$, no statistically significant statements can be made about the behavior of holes in this regime.

We now consider a magnetic polaron theory motivated by geometric strings. In the geometricstring theory, we assume that chargons are completely uncorrelated with each other, but due to their fermionic statistics Pauli blocking should actually introduce anti-correlations which have not yet been included in our analyses. We first consider a description of these chargons as free, pointlike, fermionic magnetic polarons $\hat{m}_{\mathfrak{j}}$ on a square lattice, where the known dispersion relation of the dressed hole ${ }^{\mathrm{I2} 3, \mathrm{I} 40, \mathrm{I} 4 \mathrm{I}}$ is used to define a tight-binding hopping model of the polaron. The momentum-space Hamiltonian is given by $\hat{\mathcal{H}}_{\mathrm{mp}}=\sum_{\mathrm{k}} \hat{m}_{\mathrm{k}}^{\dagger} \hat{h}_{\mathrm{k}} \epsilon_{\mathrm{mp}}(\mathrm{k})$, with approximated dispersion relation:

$$
\epsilon_{\mathrm{mp}}(\mathrm{k})=\left[4 \chi^{2} J^{2}\left|\cos \left(k_{x}\right) e^{-i \Phi / 4}+\cos \left(k_{y}\right) e^{i \Phi / 4}\right|^{2}+B_{\mathrm{st}}^{2} / 4\right]^{1 / 2}
$$

motivated by the mean-field description of the staggered flux plus Néel state ${ }^{\mathrm{I} 42}$ with parameters $\chi=$ $0.8, B_{\text {st }}=0.35 J$, and $\Phi=0.4 \pi$ determined such that the exact quantum Monte Carlo results at $J=0.4 t^{14 \mathrm{I}}$ are correctly captured. The resulting two-point correlations for a range $T=0.5 \mathrm{~J}$ to $T=0.7 J$ in the thermal state $\rho_{\mathrm{mp}}=e^{-\beta \hat{\mathcal{H}}_{\mathrm{mp}}} / Z_{\mathrm{mp}}$ are calculated for $t=2 J$. Figure $4 . \mathrm{I7c}$ shows that our data is incompatible with this model, which predicts significantly weaker hole-hole anti-correlations. Similar behavior is predicted by the $\pi$-flux theory, which models the doped holes as point-like objects moving in a quantum spin liquid of singlets.

Next we examine the picture of free chargons, motivated by considering magnetic polarons with a finite extent that results from the spinon-chargon bound state predicted by geometric-string theory. At sufficiently large chargon density, or doping, the chargons are expected to interact and their 
hard-core character will introduce anti-correlations. In this regime, geometric strings are also expected to overlap substantially and modify the dispersion relation of the chargons to be independent of spinons, yielding spinless chargons. We find that the experimental results demonstrate consistency with these free chargons, in agreement with earlier theoretical work in the strange-metal regime ${ }^{\mathrm{I} 37, \mathrm{I} 43}$. This extension to the geometric string theory may be able to explain the deviations from experiment seen in other observables at high doping, but additional analyses are required.

\subsection{Phenomenological models}

While our results indicate that both geometric strings and $\pi$-flux states predict experimental result better than sprinkled holes, it is also constructive to assess how well basic phenomenological models perform. More specifically, it continues to address the question of how restrictive these observables are; that is, how easily one can construct a dataset which matches the experimental data in all of these observables, regardless of whether that dataset is actually achievable through the Hubbard model. At the same time, the exercise of simulating a set of spin distributions which match the experimental result in all observables is certainly beyond the scope of this work, and we do not perform an exhaustive search. Now I consider two such models.

\subsection{MATCHING SPIN CORRELATIONS}

Here we begin with a random but balanced spin distribution. From this ensemble, we randomly place the desired number of doublon-hole pairs and holes according to the desired doping value. Fi-

nally, we flip spins randomly until the correlators $C_{s}(1)$ and $C_{s}(\sqrt{2})$ agree with the experimental 

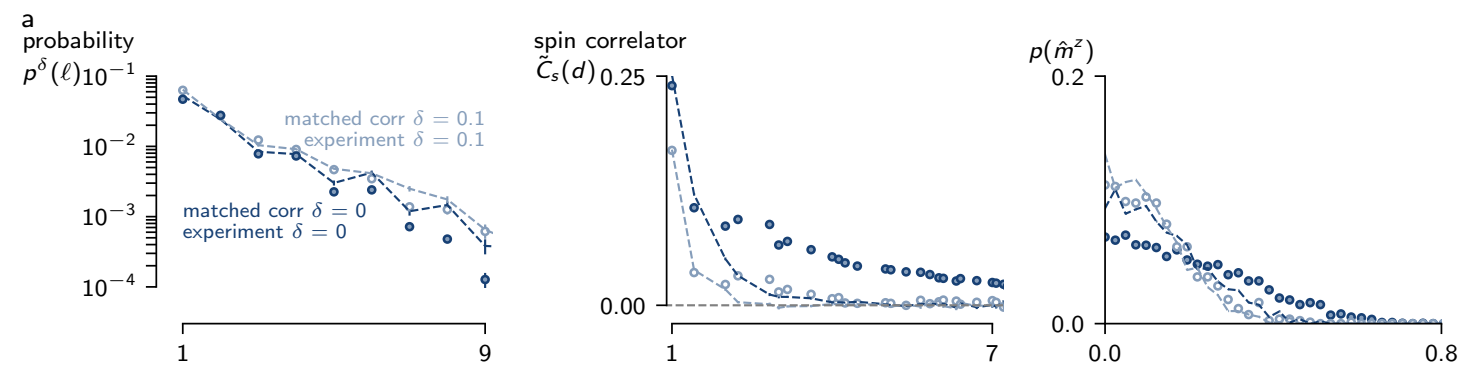

b
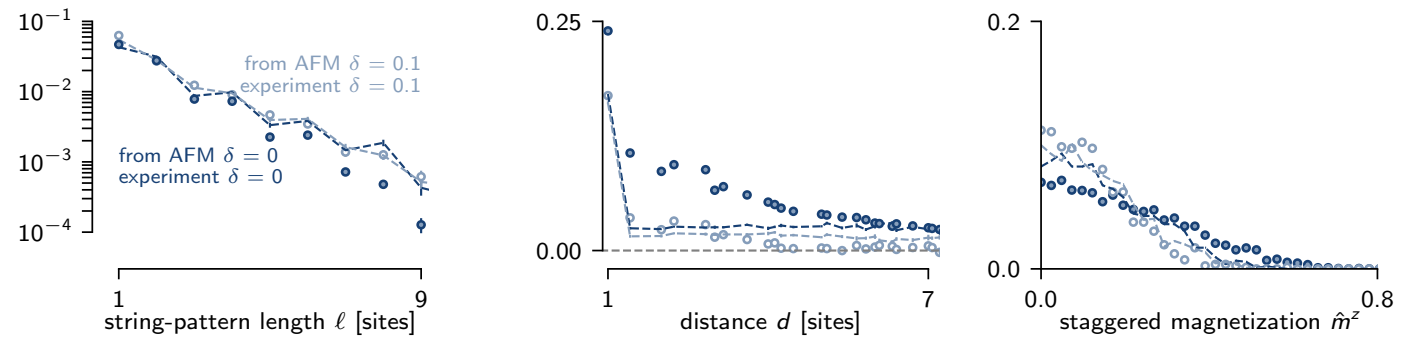

Figure 4.18: Performance of phenomenological models. a, String length histogram, spin correlation function, and staggered magnetization distribution for experiment and a phenomenological model where spins are flipped in a random spin distribution until the nearest-neighbor and next-nearest neighbor spin correlators match experimental values. b, Same quantities, but comparing experiment to a phenomenological model where singlet pairs are added to a classical Néel checkerboard and a projective measurement is performed. The singlet pair density is tuned to achieve rough agreement with the doped experimental data. For both subfigures, experimental data is shown in circles; simulated data is shown in dashed lines.

data. From this dataset, we apply our string pattern detection algorithm to compare with experimental result. The region of interest of the dataset matches that of the experiment. We generate images corresponding to half-filling and to $10 \%$ doping in experiment.

Figure 4.I8a shows the measured string pattern length distribution, spin correlation function, and full counting statistics of the staggered magnetization for the generated images in comparison to experimental result. Because we begin with spin distributions with no correlations and artificially introduce nearest-neighbor and diagonal next-nearest neighbor correlations, it is not surprising that the correlation functions do not agree beyond short distances. In turn, because the spin correlation 
function at large distance is closely related to the average staggered magnetization, it is not surprising that the staggered magnetization distribution also does not agree and that the average value is lower for the generated data.

However, the string pattern length distributions do not match either. While there is agreement at short string pattern lengths, the generated images contain statistically significantly more long patterns than in the experiment, especially for half-filling. Surprisingly, it seems that matching the first two correlators is insufficient to introduce the order needed to prevent long string patterns. Modifications to the phenomenological model such as beginning from a perfect checkerboard pattern with $S U(2)$ symmetry do not increase the level of agreement. Furthermore, the additional contributions of making $C_{s}(\sqrt{2})$ match experiment (as compared to just $C_{s}(1)$ ) are small.

\subsubsection{CORRECtions to a Classical AFM}

We also apply a phenomenological approach where we begin with a classical checkerboard, create singlets with some variable density, and place doublon-hole pairs and holes randomly according to the desired doping value. We then simulate a projective measurement process and, ensuring that the region of interest is the same as in experiment, run the string search algorithm on the result.

Figure 4.I8b shows that while the density of singlets can be varied to achieve reasonable agreement for the staggered magnetization full counting statistics and string-pattern length distribution at $10 \%$ doping, the corresponding spin correlation function seems unphysically flat at distances beyond the nearest neighbor. Furthermore, it is clear that keeping the same density of singlets for half-filling results in stark disagreement across all observables. 

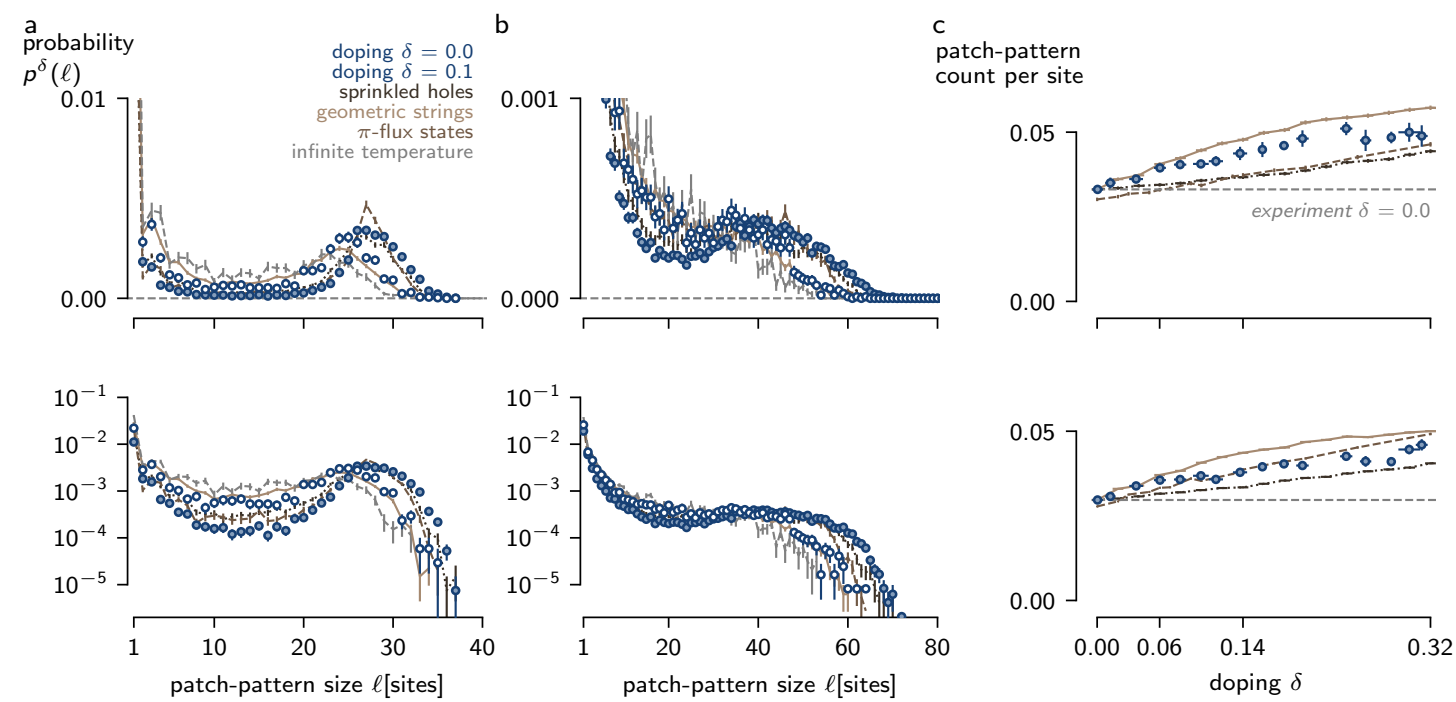

Figure 4.19: Expanding pattern detection to classical Néel patches. a, Histogram of classical Néel patch sizes as detected after our standard postselection, shown in a linear-linear plot (top) and a linear-log plot (bottom). These histograms exhibit the key features of a peak at large sizes and a plateau at more intermediate patch sizes. It also appears that the geometric strings match the experimental data best. b, Same, but without postselection. The conclusions remain unchanged. Not surprisingly, the peak in patch sizes is shifted to larger sizes and exhibits a reduced contrast. c, Total number of patch patterns with size greater than two sites, with and without postselection. The close similarities to the string-pattern count reflect a limited sensitivity of this observable to distinguishing between theories as discussed in the text. The regions of agreement and disagreement between geometric strings and experiment are consistent with that of the nearest-neighbor spin correlator and the assumption of dilute, noninteracting strings in the geometric-string theory.

\subsection{EXPANSION OF PATTERN DETECTION ALGORITHMS}

With the use of pattern detection algorithms to create new observables and compare microscopic theories, one can also begin to think about other patterns which may be able to provide insight on doped antiferromagnets. In particular, it is instructive to search for patterns which are not based upon the particular physics of one theory over others. As an example, one can consider searching for classical Néel patches: continguous regions in a snapshot which overlap with the reference checkerboard pattern. In other words, one can perform the same analysis as the simplified differ- 
ence method of section 4.5.I, but instead use the sites which match the reference checkerboard rather than the sites which deviate. These classical Néel patches can then be grouped by size and number, much like the string-pattern length histogram and string-pattern count. Furthermore, these patch patterns may also reveal information which cannot be straightforwardly captured solely by two- and multi-point correlation functions. For example, a snapshot with a given value of the staggered magnetization can realize that value through different distributions and sizes of patches which match and deviate from the reference checkerboard. Similarly, while the characteristic correlation length dictates the average length scale of spin correlations across the system, it does not capture the patch size distribution.

Figure 4.19a shows the preliminary results of the such an analysis, where the datasets are the same as described in section 4.3.I and images are postselected as before and as described in section 4.3.I. Already at half-filling (filled circles) the resulting patch size histogram exhibits a structure much different than that of the string patterns. Notably, there is a local maximum in patch size at approximately 28 sites, which is an appreciable portion of the entire 37 -site window. The patch size histogram also exhibits a maximum at small patch sizes, which may result from finite size effects. As the sample is doped (hollow circles), the peak in patch size shifts to smaller sizes, reflecting a loss of antiferromagnetic order. For a doping of $10.1(8) \%$, this peak shifts down to a patch size of approximately 26 sites, with a corresponding decrease in the number of larger patches detected and an increase in the number of smaller patches detected. This behavior is not captured by the sprinkled hole dataset, which shows some change relative to the experimental half doping dataset but not nearly enough to explain the experimental doping of $10 \%$. By contrast, the classical Néel 
patches extracted from the geometric string dataset have a size histogram which matches that of the experimental doped dataset much more closely. The agreement is not exact, with a slight deficit at large patch sizes and an excess at intermediate patch sizes between about 4 and 20 sites. Very interestingly, however, $\pi$-flux states exhibit a size histogram which continues to be peaked at a patch size of 28 sites and does not match the experimental doped dataset, showing closer resemblance to the sprinkled holes patch-size histogram.

The range of doping values to which this observable is sensitive also seems to extend beyond that of the string patterns; upon considering the "infinite-temperature" case, the peak patch size shifts down even further to a size of approximately 24 sites and the peak contrast decreases even further. The persistence of a peak may result from the nonzero strength of the nearest neighbor spin correlator $\tilde{C}_{s}(1) \approx 0.1$ for this dataset, which is actually the high-temperature dataset which spans between temperatures of $1.3(1) J$ and $1.8(1) J$. For a random distribution of spins with zero correlations at all distances, however, the patch-size histogram looks remarkably similar. The peak continues to persist and is only slightly smaller than that of the infinite temperature case, suggesting a non-negligible contribution from finite size effects. This random-spin dataset also exhibits a plateau between Io and 25 sites. Therefore, it seems here that the random-spin dataset or infinitetemperature dataset should be considered as a baseline, to which all others should be compared.

In addition to the classical Néel patch size histogram, we consider the total number of patches extracted as seen in figure 4.I9a. The experimental result looks remarkably similar to the string-pattern count histogram, which may reflect a limited usefulness of the total count in that it can be described completely by an initial slope and final saturation level. Here, too, the sprinkled holes result does 
not agree with the experimental result; neither do the $\pi$-flux states. As for geometric strings, we see agreement at low doping levels below $6 \%$, after which the prediction from geometric strings is noticeably higher than for experiment. This deviation reveals that beyond the underdoped regime there are more classical Néel patches for simulated geometric strings, which may reflect a backaction from the spin background which tends to restore antiferromagnetism. Such an interpretation would be consistent with the corresponding observed contrast in the nearest-neighbor correlator.

Because the signal to noise is particularly excellent for this pattern-based observable, it is also possible to examine the results without postselection. The results are shown in figure 4.I9b. We find they appear largely similar to the results with postselection, except that now the peak patch sizes at half-filling and $10 \%$ doping are about 45 and 35 sites, respectively. These sizes are much smaller compared to the total system size of 80 sites, relative to the case with postselection. The peak contrast has also decreased, possibly from images with poor staggered magnetization with more evenly distributed patch sizes. All datasets continue to exhibit a plateau, now between 20 and 40 sites, which is again consistent with a contribution from finite size effects. Across all simulations, the predictions from the geometric string theory continue to match the doped dataset best.

The associated patch-pattern count per site looks largely unchanged from the removal of postselection. Again, sprinkled holes do not explain the experimentally measured result, while geometric strings agree in the underdoped regime. However, the $\pi$-flux states show a stark increase in the slope of the number of patterns versus doping. It is not completely understood why this is the case, but it may indicate that although the $\pi$-flux states have been fit to match the experimental data on average, smaller subsets of snapshots may deviate. More detailed investigations are necessary to better under- 
stand these preliminary results and to better assess the preliminary conclusions here. I should also note that the idea of using pattern based observables can be extended to any type of pattern. It is not strictly necessary that these patterns have any physical motivation, however the physical interpretation of patterns which are physically motivated is significantly more intuitive.

\section{Io AdDitional Remarks}

In this chapter, I have explored how we may be able to use new observables to study the physics of strongly correlated systems. To the question of how we can extract additional useful information in our site-resolved snapshots, beyond what is contained in a two- or multi-point correlation function, we select a pattern detection algorithm to extract observables that cannot be straightforwardly expressed with correlations. These string-pattern-based observables leverage the power of quantum simulation to collapse the quantum state in a single experimental instance, and therefore represents an abstraction from and complement to established observables such as correlation functions or full counting statistics. While it is not a priori clear that this algorithm should yield observables which contain additional information, we find that this is indeed the case. More specifically, we find that certain values of the average string-pattern count and average string-pattern length can be achieved through heating an antiferromagnet but not by doping, and vice-versa.

To address how we may be able to make distinctions between candidate microscopic theories for the doped Hubbard model, we use these new observables as a complement to conventional spin observables to assess microscopic theories of the doped Hubbard model. We very carefully examine the 
effect of postselection and the string patterns found at half-filling, as well as if they have a characteristic shape and how strongly they are affected by the current spin-readout protocol, to ensure that the observables are physically meaningful. We find that much like conventional spin observables, they are sensitive at low temperatures below $T=J$ and from half-filling up to the intermediate doping regime, about $16 \%$. Across all observables considered, we find better agreement with experimental data between both the geometric-string theory and $\pi$-flux states, as compared to sprinkled holes. With no free fitting parameters, geometric strings capture the qualitative sign change of the spin correlator $\tilde{C}_{s}(\sqrt{2})$ and exhibit better quantitative agreement in the string-pattern observables, while the $\pi$-flux state shows better quantitative agreement in $\tilde{C}_{s}(1)$ but requires one free fitting parameter.

In addition, at intermediate doping values we find evidence for the onset of repulsion between holes in an antiferromagnet. Of the theoretical models considered, the experimentally measured correlations agree best with a phenomenological theory of free fermionic chargons. Further investigation is required to definitively determine whether the anticorrelation up to a distance of two lattice sites is due to quantum statistics or repulsive interactions. While signatures of other phases such as stripe phases, incommensurate spin order, or nematic fluctuations have not yet been observed in this system, they are predicted to emerge at lower temperatures. 


\section{II FUTURE DIRECTIONS}

The ideas presented in this chapter for formulating new observables can be extended to other realspace patterns, for example patterns which reflect the underlying physics of other candidate microscopic theories for the doped Hubbard model. Moreover, machine learning techniques can be used to directly compare sets of raw experimental atom distributions to theoretical models without the need for intermediate observables ${ }^{\mathrm{I} 44}$. This class of techniques is highly promising as quantum simulations of the Hubbard model continue to probe lower temperatures within the pseudogap and strange metal phases, but can also be applied to spatially resolved studies of quenches across phase transitions ${ }^{96}$, dynamical phase transitions ${ }^{145}$, and higher-order scattering processes ${ }^{146}$. Possible extensions of our work include systems with anisotropic spin interactions ${ }^{\mathrm{I2O}}$ or doped $S U(N)$ spin $\operatorname{models}^{\mathrm{I} 47}$.

Currently, in the lab we are working to characterize the dynamics of a single hole in an antiferromagnet. We can use a DMD to deterministically place a hole at a pre-determined starting site. Upon releasing the hole, we can monitor how the hole moves with time, as well as how the surrounding spin background is modified. In particular, if the hole fractionalizes into a spinon and a chargon which are bound by a geometric string, we can indirectly measure this under the microscope. Under the geometric-string theory, a hole placed in an antiferromagnetic spin background fractionalizes into a chargon and spinon, and the chargon moves ballistically at short times with a timescale on order of the tunneling energy $t$. As it moves, it displaces spins and as a result its kinetic energy becomes magnetic energy of the spin background. Once the geometric string is fully formed, the chargon 
then moves with the timescale of the spinon movement, the superexchange energy $J$. It will also be very interesting to study the motion of two holes in an antiferromagnet. Two neighboring holes may move very differently from a single hole because they do not require spins to break antialignment, but at our current temperatures it is unclear whether they will stay bound next to each other, especially since we find anticorrelations between holes. I have no doubt that in the next few years, many very interesting measurements will come out of the Greiner lab lithium quantum gas microscope as the team explores the dynamics of holes and pushes to lower temperatures. 


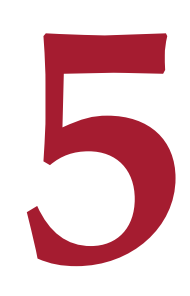

\section{Conclusion}

Over the past two years, we in the Greiner lab lithium quantum gas microscope experiment have investigated the potential for quantum simulation of the Hubbard model with ultracold atoms. After realizing a cold-atom Hubbard antiferromagnet, we created an ultra-low entropy band insulator with an entropy per particle which may be 20 times lower than that of the antiferromagnet. Using this band insulator, we explored a quantum state engineering protocol to adiabatically convert it 
into a strongly interacting many-body state. While we were ultimately unsuccessful in breaking the record $^{20}$ for the lowest temperatures achieved in a cold-atom Hubbard system, it was because we were limited by fundamental many-body physics, and even so we gained useful insights for future endeavors.

We then turned our attention to doping. In a massive experimental effort, we collected on the order of 30,000 experimental snapshots across various temperature and doping values. On top of this, we developed new observables which cannot be straightforwardly expressed in terms of two- and multi-point correlations. We found that these observables contain more information than what is captured by conventional observables such as the two-point spin correlator and staggered magnetization. By using both new and conventional observables, we see that geometric strings are a potential microscopic model for doped antiferromagnets, at least between $T=0.5 \mathrm{~J}$ and $T=0.7 \mathrm{~J}$.

At the same time, only additional experiments can reveal the predictive power of microscopic theories. As promising as our results may seem, it is much too early to judge the validity or usefulness of the geometric string theory as a microscopic spinon-chargon picture of magnetic polarons in the Hubbard model. Not only are continued comparisons necessary for lower temperatures, additional experiments are required to probe the dynamics and excitation spectra of geometric strings.

As of this writing, we are working to push forward on both of these fronts: reaching lower temperatures and better characterizing the complex interplay of spin and charge in the $2 \mathrm{D}$ Hubbard model.

Now, having worked during my graduate career to push the capabilities of quantum gas microscopes, I will add my voice to the chorus (of predominantly others also in the Fermi quantum gas 
microscope community) expressing excitement for the future of this subfield. With so many experiments online and even more on the way, the concerted effort to probe new physics in the Hubbard model is sure to pay off. In particular, I think the community will begin to address open questions on whether the Hubbard model captures the physics of the cuprates in the doped antiferromagnet and strange metal regimes. We will also continue to develop techniques for measuring spin and charge transport, such that once we reach lower temperatures we will be able to characterize and compare the low-temperature states realized. Machine learning has also recently emerged as a technique for analyzing experimental snapshots ${ }^{\mathrm{I} 48, \mathrm{I} 44}$, and I see this playing a growing role complementary to analysis via standard observables and new pattern-based observables. The community will also cautiously try to reach the low temperatures required to probe pseudogap physics, search for stripe phases (which has only recently been seen outside of the superconducting regime in cuprates ${ }^{\mathrm{I} 49}$ ) and incommensurate order, and eventually probe for $d$-wave superfluidity. These endeavors are high-risk, high-reward, and will certainly capture the attention of some groups, but not all. Needless to say, this field is sure to surprise over the years to come. 


\section{References}

[I] Bednorz, J. G. \& Müller, K. A. Possible High $T_{c}$ Superconductivity in the Ba-La-Cu-O System. Z. Phys. B-Condensed Matter 64, 189-193 (1986).

[2] Hofstetter, W., Cirac, J. I., Zoller, P., Demler, E. \& Lukin, M. D. High-temperature superfluidity of fermionic atoms in optical lattices. Physical Review Letters 89, 220407 (2002).

[3] Gutzwiller, M. C. Effect of Correlation on the Ferromagnetism of Transition Metals. Physical Review Letters 10, 159-162 (1963).

[4] Kanamori, J. Electron Correlation and Ferromagnetism of Transition Metals. Progress of Theoretical Physics 30, 275-289 (1963).

[5] Hubbard, J. Electron correlations in narrow energy bands. Proceedings of the Royal Society $A$ $276,238-257(1963)$.

[6] Feynman, R. P. Simulating Physics with Computers. International Journal of Theoretical Physics 21, 467-488 (1982).

[7] Maier, T. A., Jarrell, M., Schulthess, T. C., Kent, P. R. C. \& White, J. B. Systematic Study of $d$-Wave Superconductivity in the 2D Repulsive Hubbard Model. Physical Review Letters 95, 237001 (2005).

[8] Emery, V. J. \& Kivelson, S. A. Importance of phase fluctuations in superconductors with small superfluid density. Nature 374, 434-437 (1995).

[9] Engelbrecht, J. R., Nazarenko, A., Randeria, M. \& Dagotto, E. Pseudogap above $T_{c}$ in a model with $d_{x^{2}-y^{2}}$ pairing. Physical Review B 57, 13406-13409 (1998).

[Io] Wang, Y. et al. High Field Phase Diagram of Cuprates Derived from the Nernst Effect. Physical Review Letters 88, 257003 (2002).

[II] Taillefer, L. Scattering and Pairing in Cuprate Superconductors. Annual Review of Condensed Matter Physics I, 5I-70 (2010). 
[ı2] Hüfner, S., Hossain, M. A., Damascelli, A. \& Sawatzky, G. A. Two gaps make a hightemperature superconductor? Reports on Progress in Physics 71, 06250I (2008).

[13] Gull, E., Parcollet, O. \& Millis, A. J. Superconductivity and the Pseudogap in the TwoDimensional Hubbard Model. Physical Review Letters IIo, 216405 (2013).

[14] Varma, C. M. Theory of the pseudogap state of the cuprates. Physical Review B 73, I55113 (2006).

[15] Huber, F. G. Site-Resolved Imaging with the Fermi Gas Microscope. Ph.D. thesis, Harvard University (2014).

[16] Parsons, M. F. et al. Site-Resolved Imaging of Fermionic ${ }^{6} \mathrm{Li}$ in an Optical Lattice. Physical Review Letters II4, 213002 (2015).

[17] Parsons, M. F. Probing the Hubbard model with single-site resolution. Ph.D. thesis, Harvard University (2016).

[I8] Greif, D. G. et al. Site-resolved imaging of a fermionic Mott insulator. Science 35I, 953-957 (2016).

[19] Parsons, M. F. et al. Site-resolved measurement of the spin-correlation function in the FermiHubbard model. Science 353, I253-1256 (2016).

[20] Mazurenko, A. et al. A cold-atom Fermi-Hubbard antiferromagnet. Nature 545, 462-466 (2017).

[2I] Mazurenko, A. Probing Long Range Antiferromagnetism and Dynamics in the FermiHubbard Model. Ph.D. thesis, Harvard University (2017).

[22] Chiu, C. S., Ji, G., Mazurenko, A., Greif, D. G. \& Greiner, M. Quantum State Engineering of a Hubbard System with Ultracold Fermions. Physical Review Letters I20, 24320I (2018).

[23] Chiu, C. S. et al. String patterns in the doped Hubbard model. arXiv: 1810.03584 (2018).

[24] Ho, A. F., Cazalilla, M. A. \& Giamarchi, T. Quantum simulation of the Hubbard model: The attractive route. Physical Review A 79, 033620 (2009).

[25] Cocchi, E. et al. Equation of State of the Two-Dimensional Hubbard Model. Physical Review Letters II6, I7530I (2016). 
[26] Anderson, P. W. New Approach to the Theory of Superexchange Interactions. Physical Review II5, 2-I3 (1959).

[27] Mermin, N. D. \& Wagner, H. Absence of Ferromagnetism or Antiferromagnetism in Oneor Two-Dimensional Isotropic Heisenberg Models. Physical Review Letters 17, II33-II36 (1966).

[28] Hohenberg, P. C. Existence of Long-Range Order in One and Two Dimensions. Physical Review $158,383-386$ (1967).

[29] Troyer, M. \& Wiese, U.-J. Computational Complexity and Fundamental Limitations to Fermionic Quantum Monte Carlo Simulations. Physical Review Letters 94, 17020I (2005).

[30] Cleveland, C. L. \& Medina A., R. Obtaining a Heisenberg Hamiltonian from the Hubbard model. American Journal of Physics 44, 44-46 (1976).

[31] Auerbach, A. Interacting Electrons and Quantum Magnetism (Springer, New York, 1994).

[32] Chao, K. A., Spałek, J. \& Oleś, A. M. Kinetic exchange interaction in a narrow S-band. Journal of Physics C: Solid State Physics 10, L271-L276 (1977).

[33] Haller, E. et al. Single-atom imaging of fermions in a quantum-gas microscope. Nature Physics II, 738-742 (2015).

[34] Cheuk, L. W. et al. Quantum-Gas Microscope for Fermionic Atoms. Physical Review Letters II4, I930OI (2015).

[35] Edge, G. J. A. et al. Imaging and addressing of individual fermionic atoms in an optical lattice. Physical Review A 92, 063406 (2015).

[36] Chin, C., Grimm, R., Julienne, P. \& Tiesinga, E. Feshbach resonances in ultracold gases. Reviews of Modern Physics 82, 1225-1286 (2010).

[37] Zürn, G. et al. Precise Characterization of ${ }^{6}$ Li Feshbach Resonances Using Trap-SidebandResolved RF Spectroscopy of Weakly Bound Molecules. Physical Review Letters IIO, I3530I (2013).

[38] Grimm, R., Weidemüller, M. \& Ovchinnikov, Y. B. Optical Dipole Traps for Neutral Atoms. Advances In Atomic, Molecular, and Optical Physics 42, 95-170 (2000). 
[39] Gehm, M. E. Preparation of an optically-trapped degenerate Fermi gas of ${ }^{6}$ Li: Finding the route to degeneracy. Ph.D. thesis, Duke University (2003).

[40] Jaksch, D., Bruder, C., Cirac, J. I., Gardiner, C. W. \& Zoller, P. Cold Bosonic Atoms in Optical Lattices. Physical Review Letters 8I, 3108-31II (1998).

[4I] Jördens, R. Metallic and Mott-Insulating Phases in Fermionic Quantum Gases. Ph.D. thesis, ETH Zürich (2010).

[42] Will, S. Interacting bosons and fermions in three-dimensional optical lattice potentials: From atom optics to quantum simulation. Ph.D. thesis, Johannes Gutenberg University Mainz (20II).

[43] Demarco, B. \& Jin, D. S. Onset of Fermi Degeneracy in a Trapped Atomic Gas. Science 285, 1703-1706 (1999).

[44] Ketterle, W. \& Zwierlein, M. W. Making, probing and understanding ultracold Fermi gases. In Inguscio, M., Ketterle, W. \& Salomon, C. (eds.) International School of Physics "Enrico Fermi”, I-206 (Varenna, 2008).

[45] Greiner, M., Mandel, O., Esslinger, T., Hänsch, T. W. \& Bloch, I. Quantum phase transition from a superfluid to a Mott insulator in a gas of ultracold atoms. Nature 4I5, 39-44 (2002).

[46] Bakr, W. S., Gillen, J. I., Peng, A., Fölling, S. \& Greiner, M. A quantum gas microscope for detecting single atoms in a Hubbard-regime optical lattice. Nature 462, 74-77 (2009).

[47] Sherson, J. F. et al. Single-atom-resolved fluorescence imaging of an atomic Mott insulator. Nature 467, 68-72 (2010).

[48] Jördens, R., Strohmaier, N., Günter, K., Moritz, H. \& Esslinger, T. A Mott insulator of fermionic atoms in an optical lattice. Nature 455, 204-207 (2008).

[49] Schneider, U. et al. Metallic and Insulating Phases of Repulsively Interacting Fermions in a 3D Optical Lattice. Science 322, 1520-1525 (2008).

[5o] Duarte, P. M. et al. Compressibility of a Fermionic Mott Insulator of Ultracold Atoms. Physical Review Letters II4, 070403 (2015).

[5I] Greif, D., Uehlinger, T., Jotzu, G., Tarruell, L. \& Esslinger, T. Short-Range Quantum Magnetism of Ultracold Fermions in an Optical Lattice. Science 340, 1307-1310 (2013). 
[52] Hart, R. A. et al. Observation of antiferromagnetic correlations in the Hubbard model with ultracold atoms. Nature 519, 2II-2I4 (2015).

[53] Dutta, O. et al. Non-standard Hubbard models in optical lattices: a review. Reports on Progress in Physics 78, 0660or (2015).

[54] Taie, S., Yamazaki, R., Sugawa, S. \& Takahashi, Y. An $S U(6)$ Mott insulator of an atomic Fermi gas realized by large-spin Pomeranchuk cooling. Nature Physics 8, 825-830 (2012).

[55] Hofrichter, C. et al. Direct Probing of the Mott Crossover in the $S U(N)$ Fermi-Hubbard Model. Physical Review X 6, o21030 (2016).

[56] Uehlinger, T. et al. Artificial Graphene with Tunable Interactions. Physical Review Letters III, 185307 (2013).

[57] Messer, M. et al. Exploring Competing Density Order in the Ionic Hubbard Model with Ultracold Fermions. Physical Review Letters II5, $\mathrm{II}_{53} \mathrm{O}$ (2015).

[58] Baier, S. et al. Extended Bose-Hubbard models with ultracold magnetic atoms. Science 352, 20I-205 (2016).

[59] Miranda, M., Inoue, R., Okuyama, Y., Nakamoto, A. \& Kozuma, M. Site-resolved imaging of ytterbium atoms in a two-dimensional optical lattice. Physical Review A 91, 063414 (2015).

[6o] Yamamoto, R., Kobayashi, J., Kuno, T., Kato, K. \& Takahashi, Y. An ytterbium quantum gas microscope with narrow-line laser cooling. New Journal of Physics I8, 023016 (2016).

[6I] Omran, A. et al. Microscopic Observation of Pauli Blocking in Degenerate Fermionic Lattice Gases. Physical Review Letters II5, $26300 \mathrm{I}$ (2015).

[62] Brown, P. T. et al. Spin-imbalance in a 2D Fermi-Hubbard system. Science 357, 1385-1388 (2017).

[63] Yang, H. et al. Visualizing electronic structures of quantum materials by angle-resolved photoemission spectroscopy. Nature Reviews Materials 3, 341-353 (2018).

[64] Damascelli, A., Hussain, Z. \& Shen, Z.-X. Angle-resolved photoemission studies of the cuprate superconductors. Reviews of Modern Physics 75, 473-54I (2003). 
[65] Lee, W. S., Vishik, I. M., Lu, D. H. \& Shen, Z.-X. A brief update of angle-resolved photoemission spectroscopy on a correlated electron system. Journal of Physics: Condensed Matter 2I, 164217 (2009).

[66] Golden, M. S. et al. The electronic structure of cuprates from high energy spectroscopy. Journal of Electron Spectroscopy and Related Phenomena II7-II8, 203-222 (200I).

[67] Schmitt, F. et al. Transient Electronic Structure and Melting of a Charge Density Wave in $\mathrm{TbTe}_{3}$. Science 321, 1649-1652 (2008).

[68] Boschini, F. et al. Collapse of superconductivity in cuprates via ultrafast quenching of phase coherence. Nature Materials 17, 416-420 (2018).

[69] Ament, L. J. P., van Veenendaal, M., Devereaux, T. P., Hill, J. P. \& van den Brink, J. Resonant inelastic $\mathrm{x}$-ray scattering studies of elementary excitations. Reviews of Modern Physics 83, $705-763$ (2011).

[70] Cao, Y. et al. Ultrafast dynamics of spin and orbital correlations in quantum materials: an energy-and momentum-resolved perspective. Philosophical Transactions of the Royal Society A 377, 20170480 (2019).

[7I] Hoffman, J. E. A Search for Alternative Electronic Order in the High Temperature Superconductor $\mathrm{Bi}_{2} \mathrm{Sr}_{2} \mathrm{CaCu}_{2} \mathrm{O}_{8+\delta}$ by Scanning Tunneling Microscopy. Ph.D. thesis, University of California, Berkeley (2003).

[72] Petersen, L. et al. Direct imaging of the two-dimensional Fermi contour: Fourier-transform STM. Physical Review B 57, 6858-686I (1998).

[73] Simon, L., Bena, C., Vonau, F., Cranney, M. \& Aubel, D. Fourier-transform scanning tunnelling spectroscopy: the possibility to obtain constant-energy maps and band dispersion using a local measurement. Journal of Physics D: Applied Physics 44, 464010 (2011).

[74] van Houselt, A. \& Zandvliet, H. J. W. Colloquium: Time-resolved scanning tunneling microscopy. Reviews of Modern Physics 82, 1593-1605 (2010).

[75] Shigekawa, H., Takeuchi, O. \& Aoyama, M. Development of femtosecond time-resolved scanning tunneling microscopy for nanoscale science and technology. Science and Technology of Advanced Materials 6, 582-588 (2005). 
[76] Boll, M. et al. Spin-and density-resolved microscopy of antiferromagnetic correlations in Fermi-Hubbard chains. Science 353, I257-I260 (2016).

[77] Salomon, G. et al. Direct observation of incommensurate magnetism in Hubbard chains. Nature 565, 56-60 (2019).

[78] Hilker, T. A. et al. Revealing hidden antiferromagnetic correlations in doped Hubbard chains via string correlators. Science 357, 484-487 (2017).

[79] Nichols, M. A. et al. Spin transport in a Mott insulator of ultracold fermions. Science 363 , $383-387$ (2019).

[8o] Brown, P. T. et al. Bad metallic transport in a cold atom Fermi-Hubbard system. Science 363 , 379-382 (2019).

[8I] Brown, P. T. et al. Angle-resolved photoemission spectroscopy of a Fermi-Hubbard system. arXiv: 1903.05678 (2019).

[82] Schuster, T. A Novel Apparatus for Experiments with Ultracold Sodium and Lithium. Masters, University of Heidelberg (2008).

[83] Huber, F. G. A Novel Apparatus for Experiments with Ultracold Fermions. Masters, Harvard; Max-Planck-Institute for Quantum Optics (2009).

[84] Setiawan, W. Fermi Gas Microscope. Ph.D. thesis, Harvard University (2012).

[85] Blatt, S. et al. Low-noise optical lattices for ultracold ${ }^{6}$ Li. Physical Review A 92, 021402(R) (2015).

[86] Mazurenko, A. et al. Implementation of a stable, high-power optical lattice for quantum gas microscopy. Review of Scientific Instruments 90, 03310I (2019).

[87] Cheuk, L. W. et al. Observation of spatial charge and spin correlations in the 2D FermiHubbard model. Science 353, I260-1264 (2016).

[88] Khatami, E. \& Rigol, M. Thermodynamics of strongly interacting fermions in twodimensional optical lattices. Physical Review A 84, 0536II (201I).

[89] Pollet, L., Prokof'ev, N. V. \& Svistunov, B. V. Criticality in Trapped Atomic Systems. Physical Review Letters 104, 245705 (2010). 
[90] Bloch, I., Dalibard, J. \& Zwerger, W. Many-body physics with ultracold gases. Reviews of Modern Physics 80, 885-964 (2008).

[91] Esslinger, T. Fermi-Hubbard Physics with Atoms in an Optical Lattice. Annual Reviews of Condensed Matter Physics 1, 129-152 (2010).

[92] Roos, C. et al. Quantum State Engineering on an Optical Transition and Decoherence in a Paul Trap. Physical Review Letters 83, 4713-4716 (1999).

[93] Islam, R. et al. Onset of a quantum phase transition with a trapped ion quantum simulator. Nature Communications 2, I-6 (2011).

[94] Simon, J. et al. Quantum simulation of antiferromagnetic spin chains in an optical lattice. Nature 472, 307-312 (2011).

[95] Labuhn, H. et al. Single-atom addressing in microtraps for quantum-state engineering using Rydberg atoms. Physical Review A 90, 023415 (2014).

[96] Bernien, H. et al. Probing many-body dynamics on a 5I-atom quantum simulator. Nature 551, 579-584 (2017).

[97] Bernier, J.-S. et al. Cooling fermionic atoms in optical lattices by shaping the confinement. Physical Review A 79, 06160I(R) (2009).

[98] Ho, T.-L. \& Zhou, Q. Universal Cooling Scheme for Quantum Simulation. arXiv: ogII.5506 (2009).

[99] Lubasch, M., Murg, V., Schneider, U., Cirac, J. I. \& Bañuls, M.-C. Adiabatic Preparation of a Heisenberg Antiferromagnet Using an Optical Superlattice. Physical Review Letters 107, I6530I (2011).

[roo] Bakr, W. S. et al. Probing the Superfluid-to-Mott Insulator Transition at the Single-Atom Level. Science 329, 547-550 (2010).

[IoI] Fubini, A., Falci, G. \& Osterloh, A. Robustness of adiabatic passage through a quantum phase transition. New Journal of Physics 9, 134 (2007).

[102] Cincio, L., Dziarmaga, J., Meisner, J. \& Rams, M. M. Dynamics of a quantum phase transition with decoherence: Quantum Ising chain in a static spin environment. Physical Review B 79, 09442I (2009). 
[IO3] Hoang, T. M. et al. Adiabatic quenches and characterization of amplitude excitations in a continuous quantum phase transition. $P N A S$ II3, 9475-9479 (2016).

[104] Kantian, A., Langer, S. \& Daley, A. J. Dynamical Disentangling and Cooling of Atoms in Bilayer Optical Lattices. Physical Review Letters 120, 06040I (2018).

[105] Lee, P. A., Nagaosa, N. \& Wen, X.-G. Doping a Mott insulator: Physics of high-temperature superconductivity. Reviews of Modern Physics 78, 17-85 (2006).

[Io6] Greif, D. G. Quantum Magnetism with Ultracold Fermions in an Optical Lattice. Ph.D. thesis, ETH Zurich (2013).

[107] Cheuk, L. W. et al. Observation of 2D Fermionic Mott Insulators of ${ }^{40} \mathrm{~K}$ with Single-Site Resolution. Physical Review Letters II6, 235301 (2016).

[ro8] Zaanen, J., Osman, O. Y., Kruis, H. V., Nussinov, Z. \& Tworzydlo, J. The geometric order of stripes and Luttinger liquids. Philosophical Magazine B 81, I485-153I (200I).

[109] LeBlanc, J. P. F. et al. Solutions of the Two-Dimensional Hubbard Model: Benchmarks and Results from a Wide Range of Numerical Algorithms. Physical Review X 5, 04104I (2015).

[iro] Wen, X.-G. \& Lee, P. A. Theory of Underdoped Cuprates. Physical Review Letters 76, 503-506 (1996).

[III] Ribeiro, T. C. \& Wen, X.-G. Doped carrier formulation and mean-field theory of the $t t^{\prime} t^{\prime \prime} J$ model. Physical Review B 74, 155113 (2006).

[II2] Anderson, P. W. The Resonating Valence Bond State in $\mathrm{La}_{2} \mathrm{CuO}_{4}$ and Superconductivity. Science 235, I196-I198 (1987).

[II3] Marston, J. B. \& Affleck, I. Large- $n$ limit of the Hubbard-Heisenberg model. Physical Review B 39, II538-II558 (1989).

[II4] Lee, P. A. From high temperature superconductivity to quantum spin liquid: progress in strong correlation physics. Reports on Progress in Physics 71, 01250I (2008).

[II5] Bulaevskii, L. N., Nagaev, É. L. \& Khomskii, D. I. A new type of auto-localized state of a conduction electron in an antiferromagnetic semiconductor. Journal of Experimental and Theoretical Physics 27, 836-838 (1968). 
[II6] Brinkman, W. F. \& Rice, T. M. Single-Particle Excitations in Magnetic Insulators. Physical Review B 2, 1324-I338 (1970).

[II7] Béran, P., Poilblanc, D. \& Laughlin, R. B. Evidence for composite nature of quasiparticles in the 2D t-J model. Nuclear Physics B 473, 707-720 (1996).

[II8] Grusdt, F., Bohrdt, A. \& Demler, E. Microscopic spinon-chargon theory of magnetic polarons in the $t-J$ model. arXiv: Igor.oIII3 (2019).

[II9] Trugman, S. A. Interaction of holes in a Hubbard antiferromagnet and high-temperature superconductivity. Physical Review B 37, 1597-1603 (1988).

[120] Grusdt, F. et al. Parton Theory of Magnetic Polarons: Mesonic Resonances and Signatures in Dynamics. Physical Review X 8, 011046 (2018).

[I2I] Dagotto, E., Joynt, R., Moreo, A., Bacci, S. \& Gagliano, E. Strongly correlated electronic systems with one hole: Dynamical properties. Physical Review B 4I, 9049-4073 (1990).

[122] Liu, Z. \& Manousakis, E. Spectral function of a hole in the $t-J$ model. Physical Review B 44, 24I4-2417 (1991).

[123] Martínez, G. \& Horsch, P. Spin polarons in the $t$-J model. Physical Review B 44, 317-331 (1991).

[124] Liu, Z. \& Manousakis, E. Dynamical properties of a hole in a Heisenberg antiferromagnet. Physical Review B 45, 2425-2437 (1992).

[125] Mishchenko, A. S., Prokof'ev, N. V. \& Svistunov, B. V. Single-hole spectral function and spin-charge separation in the $t-J$ model. Physical Review B 64, 03310I (200I).

[126] Lieb, E. H. \& Wu, F. Y. Absence of Mott Transition in an Exact Solution of the Short-Range, One-Band Model in One Dimension. Physical Review Letters 20, I445-I448 (1968).

[127] Woynarovich, F. Excitations with complex wavenumbers in a Hubbard chain: I. States with one pair of complex wavenumbers. Journal of Physics $C$ 15, 85-96 (1982).

[128] Ogata, M. \& Shiba, H. Bethe-ansatz wave function, momentum distribution, and spin correlation in the one-dimensional strongly correlated Hubbard model. Physical Review B 4I, $2326-2338$ (1990). 
[129] Kruis, H. V., McCulloch, I. P., Nussinov, Z. \& Zaanen, J. Geometry and the hidden order of Luttinger liquids: The universality of squeezed space. Physical Review B 70, 075109 (2004).

[130] Endres, M. et al. Observation of Correlated Particle-Hole Pairs and String Order in LowDimensional Mott Insulators. Science 334, 200-203 (20II).

[13I] Paiva, T., Scalettar, R., Randeria, M. \& Trivedi, N. Fermions in 2D Optical Lattices: Temperature and Entropy Scales for Observing Antiferromagnetism and Superfluidity. Physical Review Letters 104, 066406 (2010).

[132] Varney, C. N. et al. Quantum Monte Carlo study of the two-dimensional fermion Hubbard model. Physical Review B 80, 075116 (2009).

[133] Gros, C. Physics of Projected Wavefunctions. Annals of Physics I89, 53-88 (1989).

[134] Gorelik, E. V. et al. Universal probes for antiferromagnetic correlations and entropy in cold fermions on optical lattices. Physical Review A 85, 061602 (2012).

[135] Keimer, B., Kivelson, S. A., Norman, M. R., Uchida, S. \& Zaanen, J. From quantum matter to high-temperature superconductivity in copper oxides. Nature 518, I79-I86 (2015).

[136] White, S. R. \& Scalapino, D. J. Density Matrix Renormalization Group Study of the Striped Phase in the 2D t-J Model. Physical Review Letters 80, I272-1275 (1998).

[137] Kaul, R. K., Kim, Y. B., Sachdev, S. \& Senthil, T. Algebraic charge liquids. Nature Physics 4, 28-3I (2008).

[138] Kane, C. L., Lee, P. A. \& Read, N. Motion of a single hole in a quantum antiferromagnet. Physical Review B 39, 6880-6897 (1989).

[139] Sachdev, S. Hole motion in a quantum Néel state. Physical Review B 39, I2232-I2247 (1989).

[I40] Liu, J., Sun, X., Lin, D. L. \& Georgy, T. F. Gap states of polaron and associated optical absorption in the quasi-one-dimensional solid $\left[\mathrm{Pt}(\mathrm{en})_{2}\right]\left[\mathrm{PtCl}_{2}(\mathrm{en})_{2}\right]\left(\mathrm{ClO}_{4}\right)_{4}$. Journal of Physics: Condensed Matter 4, 530I-5308 (1992).

[I4I] Brunner, M., Assaad, F. F. \& Muramatsu, A. Single-hole dynamics in the $t-J$ model on a square lattice. Physical Review B 62, 15480-15492 (2000).

[I42] Lee, T. K. \& Feng, S. Doping dependence of antiferromagnetism in $\mathrm{La}_{2} \mathrm{CuO}_{4}$ : A numerical study based on a resonating-valence-bond state. Physical Review B 38, II809-II8I2 (I988). 
[I43] Sachdev, S. \& Chowdhury, D. The novel metallic states of the cuprates: Topological Fermi liquids and strange metals. Progress of Theoretical and Experimental Physics 2016, ${ }_{2} \mathrm{Cro} 2$ (2016).

[144] Bohrdt, A. et al. Classifying Snapshots of the Doped Hubbard Model with Machine Learning. arXiv I8II.12425 (2018).

[145] Zhang, J. et al. Observation of a many-body dynamical phase transition with a 53-qubit quantum simulator. Nature 551, 60I-604 (2017).

[146] Feng, L., Hu, J., Clark, L. W. \& Chin, C. Correlations in high-harmonic generation of matterwave jets revealed by pattern recognition. Science 363, 521-524 (2019).

[I47] Honerkamp, C. \& Hofstetter, W. Ultracold Fermions and the $S U(N)$ Hubbard Model. Physical Review Letters 92, 170403 (2004).

[148] Rem, B. S. et al. Identifying Quantum Phase Transitions using Artificial Neural Networks on Experimental Data. arXiv: I809.05519 (2018).

[149] Zhao, H. et al. Charge-stripe crystal phase in an insulating cuprate. Nature Materials i8, I03-107 (2019). 


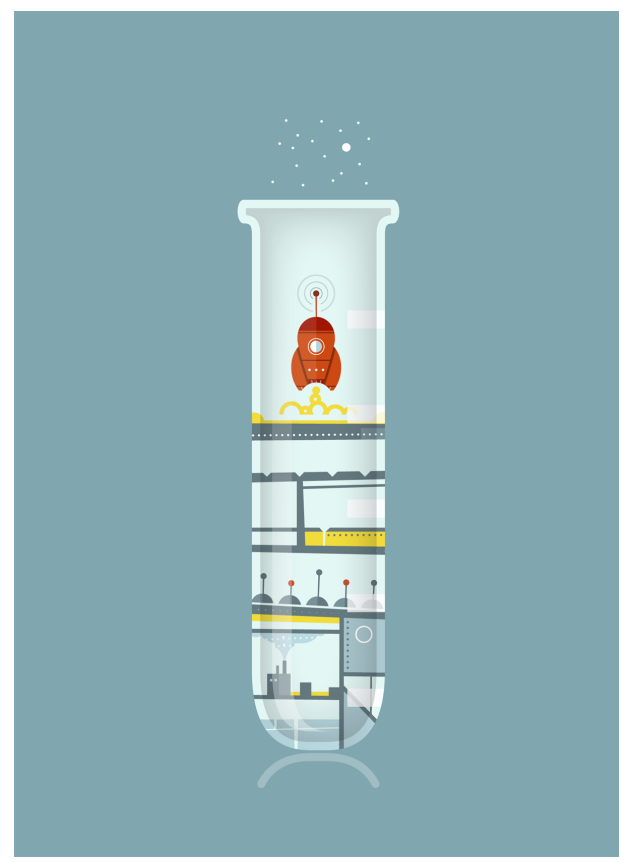

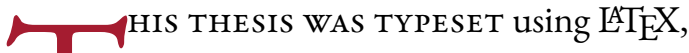 originally developed by Leslie Lamport and based on Donald Knuth's TEX.

The body text is set in In point Egenolff-Berner Garamond, a revival of Claude Garamont's humanist typeface. The above illustration, Science Experiment 02, was created by Ben Schlitter and released under CC BY-NC-ND 3.O. A template that can be used to format a $\mathrm{PhD}$ dissertation with this look $\mathbb{E}$ feel has been released under the permissive AGPL license, and can be found online at github.com/suchow/Dissertate or from its lead author, Jordan Suchow, at suchow@post.harvard.edu. 